\title{
Coronal Mass Ejections: Models and Their Observational Basis
}

\author{
P. F. Chen \\ Department of Astronomy, Nanjing University \\ Nanjing 210093, China \\ email: chenpf@nju.edu.cn \\ http://astronomy.nju.edu.cn/ chenpf/
}

Accepted on 3 March 2011

Published on 8 April 2011

\begin{abstract}
Coronal mass ejections (CMEs) are the largest-scale eruptive phenomenon in the solar system, expanding from active region-sized nonpotential magnetic structure to a much larger size. The bulk of plasma with a mass of $\sim 10^{11}-10^{13} \mathrm{~kg}$ is hauled up all the way out to the interplanetary space with a typical velocity of several hundred or even more than $1000 \mathrm{~km} \mathrm{~s}^{-1}$, with a chance to impact our Earth, resulting in hazardous space weather conditions. They involve many other much smaller-sized solar eruptive phenomena, such as X-ray sigmoids, filament/prominence eruptions, solar flares, plasma heating and radiation, particle acceleration, EIT waves, EUV dimmings, Moreton waves, solar radio bursts, and so on. It is believed that, by shedding the accumulating magnetic energy and helicity, they complete the last link in the chain of the cycling of the solar magnetic field. In this review, I try to explicate our understanding on each stage of the fantastic phenomenon, including their pre-eruption structure, their triggering mechanisms and the precursors indicating the initiation process, their acceleration and propagation. Particular attention is paid to clarify some hot debates, e.g., whether magnetic reconnection is necessary for the eruption, whether there are two types of CMEs, how the CME frontal loop is formed, and whether halo CMEs are special.
\end{abstract}

This review is licensed under a Creative Commons Attribution-Non-Commercial-NoDerivs 3.0 Germany License. http://creativecommons.org/licenses/by-nc-nd/3.0/de/ 


\section{Imprint / Terms of Use}

Living Reviews in Solar Physics is a peer reviewed open access journal published by the Max Planck Institute for Solar System Research, Max-Planck-Str. 2, 37191 Katlenburg-Lindau, Germany. ISSN 1614-4961.

This review is licensed under a Creative Commons Attribution-Non-Commercial-NoDerivs 3.0 Germany License: http://creativecommons.org/licenses/by-nc-nd/3.0/de/

Because a Living Reviews article can evolve over time, we recommend to cite the article as follows:

\section{P. F. Chen,}

"Coronal Mass Ejections: Models and Their Observational Basis", Living Rev. Solar Phys., 8, (2011), 1. [Online Article]: cited [<date $>$ ], http://www.livingreviews.org/lrsp-2011-1

The date given as $<$ date $>$ then uniquely identifies the version of the article you are referring to.

\section{Article Revisions}

Living Reviews supports two ways of keeping its articles up-to-date:

Fast-track revision A fast-track revision provides the author with the opportunity to add short notices of current research results, trends and developments, or important publications to the article. A fast-track revision is refereed by the responsible subject editor. If an article has undergone a fast-track revision, a summary of changes will be listed here.

Major update A major update will include substantial changes and additions and is subject to full external refereeing. It is published with a new publication number.

For detailed documentation of an article's evolution, please refer to the history document of the article's online version at http: //www.livingreviews.org/lrsp-2011-1. 


\section{Contents}

1 Introduction $\quad 5$

2 Observational Features $\quad 6$

2.1 Morphology and mass . . . . . . . . . . . . . . . . . . . . . 6

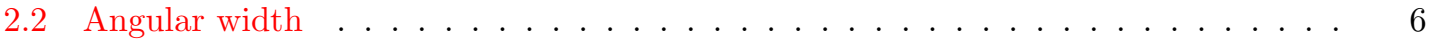

2.3 Occurrence rate . . . . . . . . . . . . . . . . . . . . . . . . 7

2.4 Velocity and energy . . . . . . . . . . . . . . . . . . . . . 8

2.5 Association with flares and filament eruptions . . . . . . . . . . . . . 8

3 Theoretical Models 10

3.1 Basic principles . . . . . . . . . . . . . . . . . . . . . . . . 10

3.2 Global picture . . . . . . . . . . . . . . . . . . . . . . . . . . . . . . . . . . .

3.3 Progenitor . . . . . . . . . . . . . . . ......... 13

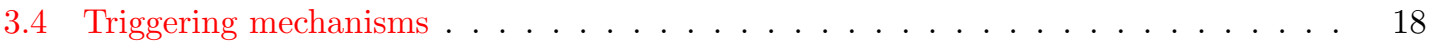

3.4.1 Observational precursors . . . . . . . . . . . . . . . . . 18

3.4.2 Various triggering mechanisms . . . . . . . . . . . . . . . . . . 23

3.4 .3 Remarks for the progenitors . . . . . . . . . . . . . . . . . 37

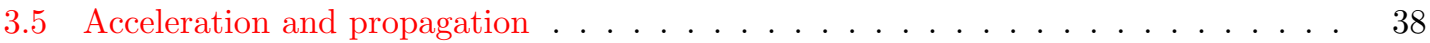

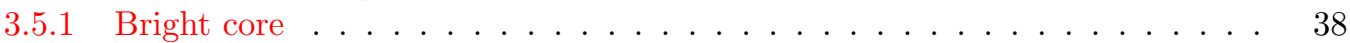

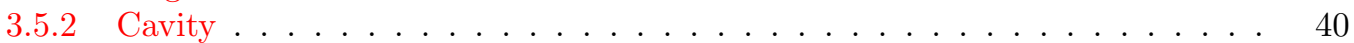

3.5.3 Frontal loop . . . . . . . . . . . . . . . . . . . . . 42

3.5.4 Lateral expansion . . . . . . . . . . . . . . . . . . . . . . . . . . . . . 44

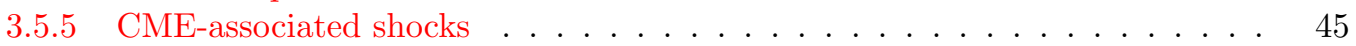

4 Debates $\quad \mathbf{4 8}$

4.1 Is magnetic reconnection necessary? . . . . . . . . . . . . . . . . . . . . . . . . . . 48

4.2 Should fast and slow CMEs be attributed to different models? . . . . . . . . . . . . 50

4.3 Nature and the driving source of "EIT waves" . . . . . . . . . . . . . . . . . 53

4.3.1 Fast-mode MHD wave model . . . . . . . . . . . . . . . . . . . . 54

4.3.2 Magnetic fieldline successive stretching model . . . . . . . . . . . . . . . . 55

4.3.3 Successive reconnection model . . . . . . . . . . . . . . . . 56

4.3 .4 Current shell model . . . . . . . . . . . . . . . . . . . . 57

4.3.5 Slow-mode wave or soliton wave model . . . . . . . . . . . . . . 57

4.4 What is the nature of CMEs? . . . . . . . . . . . . . . . . . . . . . . . . . . . . . . . . . . . . .

4.5 Are halo CMEs special? . . . . . . . . . . . . . . . . . . . 61

5 Summary $\quad 63$

6 Acknowledgements $\quad 65$

$\begin{array}{lr}\text { References } & 66\end{array}$

\section{List of Tables}

1 Estimates of the coronal energy sources. . . . . . . . . . . . . . . . . 



\section{Introduction}

Coronal mass ejections (CMEs) are spectacular eruptions in the solar atmosphere. They originate from coronal-loop-sized scale $\left(\sim 10^{4} \mathrm{~km}\right)$, expand to cover a significant part of the solar surface, and further extend all the way from the low corona to the interplanetary space, through which they become the largest-scale eruptive phenomenon in the solar system. Similar phenomenon was also identified on other stars (Collier Cameron and Robinson, 1989). During their propagation in the solar system, CMEs may frequently interact with the Earth (and other planets), producing a series of impacts on the terrestrial environment and the human high-tech activities (see Schwenn, 2006; Pulkkinen, 2007, and references therein). Although CMEs may have been caught a glimpse during the occasional total solar eclipses in the past thousands of years, and have been inferred in the early 20th century (see Cliver, 1995; Alexander et al., 2006, for reviews) as an eruptive phenomena, they were discovered by the coronagraph on board the seventh Orbiting Solar Observatory (OSO-7) satellite on 1971 December 14 (Tousey, 1973), which was 112 years after the first observation of solar flares. Such a delay resulted in many controversies in identifying the source of various shock wave-related phenomena, e.g., type II radio bursts, Moreton waves, and EIT waves ${ }^{1}$ (Vršnak and Cliver, 2008), as well as the source of the ensuring space weather disturbances (Gosling, 1993). On the other hand, the understanding of CMEs benefits significantly from the earlier developed models for solar flares, as well as filament eruptions (see Priest and Forbes, 2002, for a review on solar flares).

A lot of CME-related models have been developed to describe their pre-eruption structures (hereafter called progenitors), their initiations, and their eruptions. It is found that from time to time these models, which describe different phases of the CMEs, were lumped together in the literature. In this paper, we try to separate them into different subsections in order to distinguish their applications to different stages of CMEs, which may be biased toward my personal viewpoints though. For more reviews on CME models, the readers are referred to Klimchuk (2001), Zhang and Low (2005), Forbes (2000), Forbes et al. (2006), and Vršnak (2008).

In this paper, we start with the brief description of the observational features of CMEs in Section 2, and various CME models are reviewed in Section 3, where the subsections are devoted to the models for their pre-eruption, initiation, and eruption stages. In Section 4, we present several topics that are under strong controversy or remain elusive, which are followed by a summary in Section 5 .

${ }^{1}$ EIT waves are a wave-like phenomenon discovered by the EUV Imaging Telescope (EIT) aboard the Solar and Heliospheric Observatory ( $\mathrm{SOHO}$ ) satellite (see Section 4.3 for details) 


\section{Observational Features}

According to the original definition, CMEs are an observable change in the coronal structure that involves the appearance (Hundhausen et al., 1984) and outward motion (Schwenn, 1996) of a new, discrete, bright, white-light feature in the coronagraph field of view. Further observations indicate that CMEs can also be observed in other wavelengths, such as soft X-rays (SXR, e.g., Rust, 1983; Klimchuk et al., 1994; Gopalswamy et al., 1996), extreme ultra-violet (EUV, Dere et al., 1997; Chen, 2009a), radio (Gopalswamy and Kundu, 1992; Maia et al., 1999), and so on (see Hudson and Cliver, 2001, for more details). The multiwavelength observations are thought to be crucial to a complete understanding of CMEs (Hudson and Cliver, 2001). Recently, it was proposed that Ly $\alpha$ line at the ultra-violet (UV) wavelength might be very suitable for the detection of CMEs (Vial et al., 2007).

The white-light emission of the corona comes from the photospheric radiation Thomson-scattered by free electrons in the corona, and any enhanced brightness means that the coronal density somewhere along the line of sight is increased. In addition to the density enhancement, the Thomsonscattered radiation depends on the photospheric radiation incident to the electrons and the angle between the incidence and the line of sight, which makes CMEs favorably observed near the plane of the sky. With the continual observations from various ground-based and space-born coronagraphs, more than ten thousand CME events have been recorded, which enables the statistical investigation of their properties.

\subsection{Morphology and mass}

CMEs present many different shapes, and much of the variety is believed simply due to the projection effects (Schwenn, 2006). However, fundamental difference can be found between narrow CMEs and others (sometimes called normal CMEs). The narrow CMEs show jet-like motions probably along open magnetic field, whereas normal CMEs are characterized by a closed frontal loop, as shown in Figure 1. The typical morphology for normal CMEs is the so-called three-part structure, i.e., a bright frontal loop, which is immediately followed by a dark cavity with an embedded bright core (Illing and Hundhausen, 1985). The bright core corresponds to the erupting filament (House et al., 1981).

The three-part structure is considered to be the standard morphology for CMEs, although observations indicate that only $\sim 30 \%$ of CME events possess all the three parts (Webb and Hundhausen, 1987). Among the events without a bright core, some are due to that the filament materials drained down to the solar surface along the stretched magnetic field, some are due to that thermal instability had not started to form a filament in the pre-eruption structure, and others might not be related to filament or filament-supporting structure at all.

Based on the Thomson-scattering formulae (see Billings, 1966), the mass of a CME can be estimated (Hundhausen, 1993). Without the knowledge of the exact position of the density-enhanced structure, it is often assumed that the CME is close to the plane of the sky, which would underestimate the mass of the CME, especially for halo events.

Typically, the mass of a CME falls in the range of $1 \times 10^{11}-4 \times 10^{13} \mathrm{~kg}$, averaged at $3 \times 10^{12} \mathrm{~kg}$ (Jackson, 1985; Gopalswamy and Kundu, 1992; Hudson et al., 1996). About 15\% of the CMEs have a mass less than $10^{11} \mathrm{~kg}$ (Vourlidas et al., 2002).

\subsection{Angular width}

The angular width of CMEs projected in the plane of the sky ranges widely from $\sim 2^{\circ}$ to $360^{\circ}$ (Yashiro et al., 2004), with a significant fraction in the low end (e.g., $<20^{\circ}$ ) and a small fraction in the high end (e.g., $>120^{\circ}$ ). The CMEs with the angular width less than $\sim 10^{\circ}$ can be called

Living Reviews in Solar Physics

http://www. livingreviews.org/lrsp-2011-1 


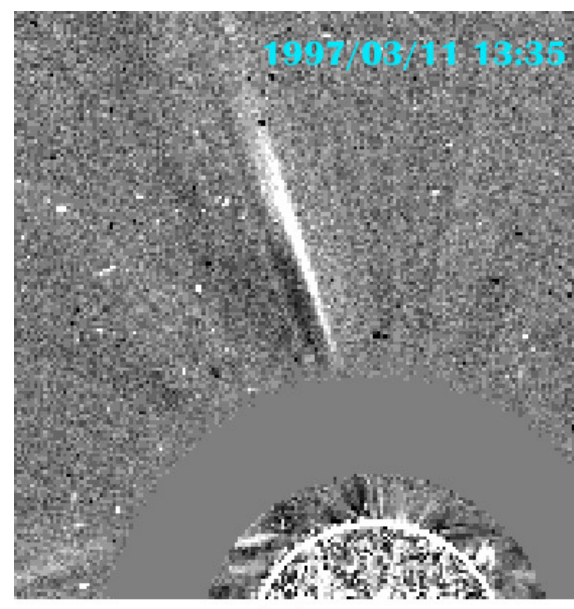

(a)

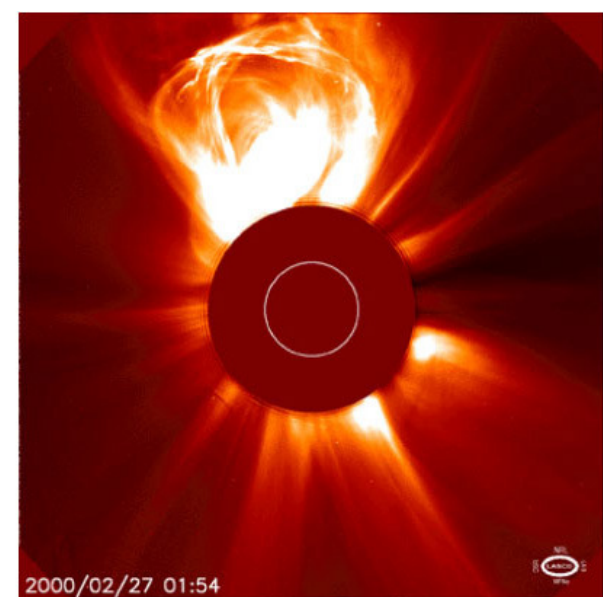

(b)

Figure 1: White-light images of two types of typical CMEs (from SOHO/LASCO database). (a) A narrow CME on 1997 March 11, where the $195 \AA$ A disk image is overplotted on the occulting disk; (b) a normal CME on 2000 February 27 with a three-part structure, i.e., a frontal loop, a cavity, and a bright core, where the white circle marks the solar limb.

narrow CMEs (Wang et al., 1998), and others are sometimes called normal CMEs (Yashiro et al., 2004, see Figure 1). Note that halo CMEs, with an apparent angular width of or close to $360^{\circ}$, are simply due to that the CMEs, probably with an angular width of tens of degrees, propagate near the Sun-Earth line, either toward or away from the Earth.

\subsection{Occurrence rate}

During the solar cycle 23, the Large Angle and Spectrometric Coronagraph (LASCO) on board the SOHO satellite provided unprecedented observations of CMEs. The occurrence rate of CMEs was found to basically track the solar activity cycle, but with a peak delay of 6-12 months (Raychaudhuri, 2005; Robbrecht et al., 2009). Before the SOHO era, the averaged occurrence rate was found to increase from 0.2 per day at solar minimum to 3.5 per day at solar maximum (Webb and Howard, 1994). With the increased sensitivity and wider field of view, the SOHO/LASCO coronagraph assembly, including $\mathrm{C} 1, \mathrm{C} 2$, and $\mathrm{C} 3$ components with different fields of view, detected CMEs more frequently. The CME catalog ${ }^{2}$ in the NASA CDAW data center, where CMEs are identified by eye, shows that the CME occurrence rate increases from $\sim 0.5$ per day near solar minimum to $\sim 6$ near solar maximum, summing up to more than 13000 CMEs during the solar cycle 23 (Gopalswamy et al., 2003; Yashiro et al., 2004). However, for the same observational period, the automated software, CACTus ${ }^{3}$, identified much more events, with the occurrence rate increasing from $<2$ per day near solar minimum to $\sim 8$ per day near solar maximum (Robbrecht et al., 2009). Figure 2 shows the comparison of the CME daily occurrence rate detected by the two methods, along with the sunspot number.

\footnotetext{
2 http://cdaw.gsfc.nasa.gov/CME_list

3 http://sidc.oma.be/cactus/scan
} 


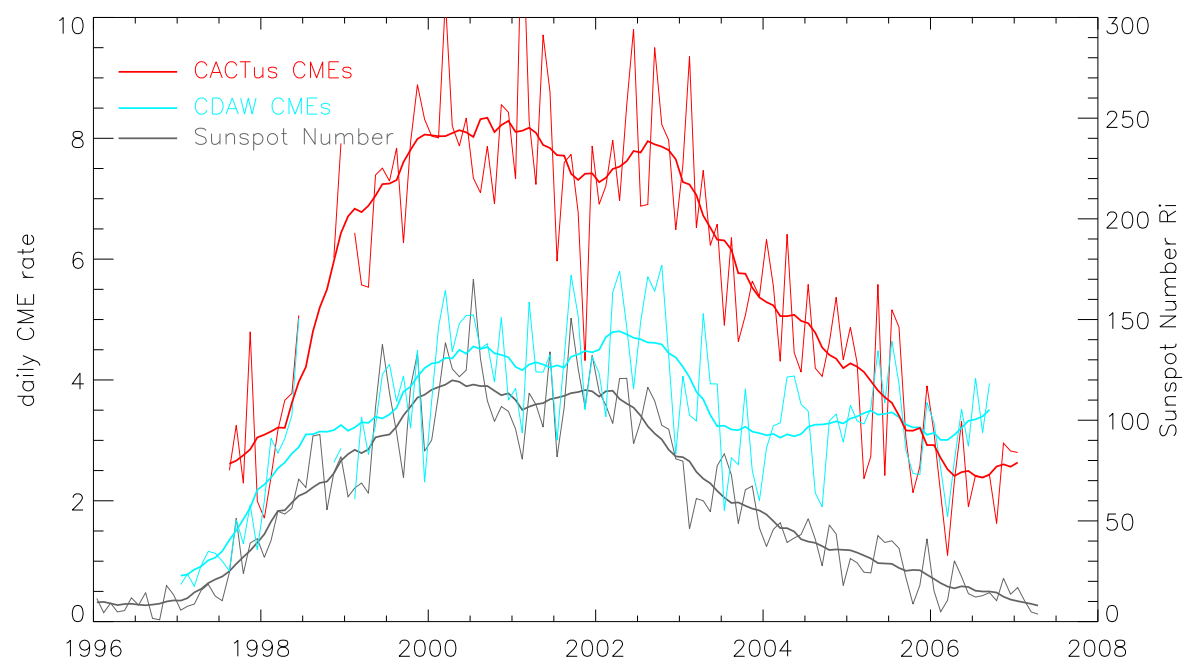

Figure 2: The CME daily occurrence rate detected by the CACTus archive (red) and the CDAW archive (blue) compared with the daily sunspot number (gray) during solar cycle 23. Thin curves: smoothed per month, thick curves: smoothed over 13 months (from Robbrecht et al., 2009).

\subsection{Velocity and energy}

Without special declaration, the CME velocity general means the radial propagation speed of the top part of a CME frontal loop. However, it should be noted that this velocity measures the motion of the CME frontal loop projected in the plane of the sky, therefore, it can be called projected velocity. There are continuous attempts trying to correct the propagation velocity for the projection effects. The CME projected velocity ranges from $\sim 20 \mathrm{~km} \mathrm{~s}^{-1}$ to $>2000 \mathrm{~km} \mathrm{~s}^{-1}$, occasionally reaching $3500 \mathrm{~km} \mathrm{~s}^{-1}$. The averaged velocity increases from $300 \mathrm{~km} \mathrm{~s}^{-1}$ near solar minimum to $500 \mathrm{~km} \mathrm{~s}^{-1}$ near solar maximum (Yashiro et al., 2004).

Two issues should be emphasized here. First, it is often taken for granted that the observed CME propagation in the coronagraph images is a mass motion, which was not judged based on spectroscopic observations. On the contrary, there is accumulating evidence to reveal that the propagation of the frontal loop in many CME events, especially those fast events, is not a mass motion. This issue is actually strongly related to the nature of CMEs (see discussions in Section 4.4). Second, all the plasmas within the CME cavity are also moving outward, not just the material along the frontal loop, as implied by spectral observations in the dimming region under the CME (Harra and Sterling, 2001).

It is found that the kinetic and potential energies of a typical CME amount to $10^{22}-10^{25} \mathrm{~J}$, which is similar to that of solar flares (Vourlidas et al., 2002; Emslie et al., 2004).

\subsection{Association with flares and filament ${ }^{4}$ eruptions}

CMEs are often accompanied by solar flares, and they are thought to be signatures of the same magnetic "disease" (Harrison, 1995). There might be some CMEs which are not associated with flares (see discussions in Section 4.2). However, it should be reminded that for some of these flareless CMEs, the lack of association may be due to: (1) the source region is behind the solar limb; (2) the associated SXR flaring arcade or disk brightenings below the CME are so weak that

\footnotetext{
${ }^{4}$ A filament is called a prominence when it appears above the limb. Therefore, hereafter, the two nomenclatures are used reciprocally.
}

Living Reviews in Solar Physics

http://www . livingreviews . org/lrsp-2011-1 
it is not registered as a flare (Hiei et al., 1993; Wu et al., 2002; Zhou et al., 2003). On the other side, many flares are not associated with CMEs. For instance, $\sim 70 \%$ of C-class, $\sim 44 \%$ of M-class, and $\sim 10 \%$ of X-class SXR flares are not associated with CMEs (Yashiro et al., 2005; Wang and Zhang, 2007).

CMEs and filament/prominence eruptions are also strongly related (Gopalswamy et al., 2003; Jing et al., 2004, and references therein), and part of the erupting filament becomes the bright core of the CME, with the remaining part falling down to the solar surface. The association rate derived from observations depends on the observational sample and the wavelength.

The strong association between CMEs and flare/filament eruptions paved the way to construct the CMEs models from the heritage of flare and filament researches. It is now believed that, when they are associated, flares and CMEs are different aspects of one global magnetic eruption (Harrison, 1995; Forbes, 2000; Zhang et al., 2001a). 


\section{Theoretical Models}

\subsection{Basic principles}

Constructing CME models is extremely important, not only because CMEs are a spectacular astronomical phenomenon, but also because they are the main driver for the space weather disturbances that strongly affect our high-tech life. It is emphasized here that any successful model should be based on the combination of observations and magnetohydrodynamic (MHD) theory.

The same as any other eruptive phenomenon, CMEs, along with solar flares, involve the energy conversion from one kind to the kinetic, potential, thermal, and nonthermal energies, as well as the radiative energy in flaring loops. It should be noted that there are secondary conversions between different energies, e.g., part of the nonthermal energy would be converted into the thermal energy, which would finally radiate out. They should not be double counted when estimating the CME and flare energies. With the assumption that a typical CME involves a volume of $10^{24} \mathrm{~m}^{3}$, the energy density of a CME ranges from $10^{-2}-10 \mathrm{~J} \mathrm{~m}^{-3}$. The typical energy density of possible energy sources is shown in Table 1 (Forbes, 2000). We can see that for energetic CME events, which are the most interesting in the space weather context, the only possible source is the magnetic energy, whereas for very weak CME events, thermal and potential energies in the pre-eruption corona may contribute to the CME explosions. In the case that these two sources are available, thermal energy is converted to the CME energy by the work of pressure gradient, similar to the acceleration of solar wind, and the potential energy is converted to the CME energy in the form of buoyancy.

Table 1: Estimates of the coronal energy sources (adapted from Forbes, 2000).

\begin{tabular}{lll}
\hline Form of energy & Energy density $\left(\mathbf{J ~ m}^{-\mathbf{3}}\right)$ & Observed averaged value \\
\hline Kinetic $\left(\frac{1}{2} m_{p} n V^{2}\right)$ & $8 \times 10^{-4}$ & $n=10^{15} \mathrm{~m}^{-3}, V=1 \mathrm{~km} \mathrm{~s}^{-1}$ \\
Thermal $(n k T)$ & $1 \times 10^{-2}$ & $T=10^{6} \mathrm{~K}$ \\
Potential $\left(n m_{p} g h\right)$ & $5 \times 10^{-2}$ & $h=10^{5} \mathrm{~km}$ \\
Magnetic $\left(B^{2} / 2 \mu_{0}\right)$ & 40 & $B=10^{-2} \mathrm{~T}$ \\
\hline
\end{tabular}

In those eruptive cases, the CMEs energy comes from the partial release of the magnetic free energy, i.e., the excess energy compared to the potential field with the same flux distribution at the solar photosphere. It is demonstrated that in the case of force-free field that is often applicable in the low corona (Gary, 2001), the magnetic free energy is of the order of the magnetic energy of the corresponding potential field (Aly, 1984). For example, Aly (1991) showed that for a simply connected field the total magnetic energy is less than twice the potential magnetic energy. In the cases when gravity is important, e.g., when a filament is present, or that the gas pressure is not negligible, the total magnetic energy can be derived from the virial theorem (Low, 1999):

$$
\int_{r>R_{0}} B^{2} /\left(2 \mu_{0}\right) \mathrm{d} V=\int_{r=R_{0}}\left[\frac{1}{2 \mu_{0}}\left(B_{r}^{2}-B_{\theta}^{2}-B_{\phi}^{2}\right)-p\right] \mathrm{d} S+\int_{r>R_{0}}\left(\frac{\rho G M_{0}}{r}-3 p\right) \mathrm{d} V,
$$

which would lead to a higher magnetic free energy. It should be noted here that due to the strong line-tying effect of the solar surface, the photospheric vector magnetogram does not transit to a potential state after the eruption. It remains to be close to a nonlinear force-free field (e.g., Jing et al., 2009; Thalmann and Wiegelmann, 2008), with a certain amount of magnetic free energy and magnetic helicity. Since the whole process is constrained by the conservation of magnetic energy and helicity (Berger, 1984; Aly, 1991), only a part of the free energy, as well as helicity, can be really released from the corona. As a consequence, SXR sigmoids, which are generally believed to be the nonpotential pre-CME structure, may form after the onset of some CMEs (Glover et al., 2001).

Living Reviews in Solar Physics

http://www. livingreviews.org/lrsp-2011-1 
Except some slow CMEs which might be accelerated by the ambient solar wind (see Section 4.2), it is well established that many CMEs are due to the rapid release of magnetic energy in the corona. However, the coronal magnetic field can not yet be measured directly. The quantitative study of the coronal magnetic field generally relies on the magnetic extrapolation based on the photospheric magnetograms. However, for a given photospheric magnetogram, the extrapolation of the coronal field is not unique, depending on the methods (DeRosa et al., 2009). More importantly, the extrapolation technique may have smoothed some singular magnetic topologies which are important for the eruptions. Besides, the magnetic field in the photosphere is probably not in a force-free state. Therefore, the understanding of CMEs relies mainly on the multiwavelength imaging and spectroscopic observations. With the following reasons, the physical processes in CMEs are far from been fully resolved: (1) white-light coronagraphs, which observe the CMEs directly, always occult the solar disk and the innermost corona where CMEs originate; (2) coronagraph observations favor the CMEs propagating near the plane of the sky, whereas the CMEs source region can be better diagnosed near the solar disk center; (3) the SXR and EUV emissions of the corona are optically thin, and it is still difficult for the spectrometers to possess both a wide field of view and a high time cadence simultaneously (see Harrison and Lyons, 2000). Despite these difficulties, much progress has been made in the past decades, along with many controversies.

\subsection{Global picture}

Many researchers hope to see a unified model which can explain the diversity of CMEs. The observations, however, seem to show that it is necessary to divide CMEs into at least two categories, i.e., narrow CMEs and normal CMEs. The former has an angular width of $\sim 10^{\circ}$ and the latter has a larger angular width. Note that there is no dividing line in the angular width between the two groups. This grouping is apparently based on the angular width. However, the essential feature that can distinguish them is that narrow CMEs show an elongated jet-like shape, whereas normal CMEs present a closed (or convex-outward) loop, as shown by Figure 1. It is noticed that many CMEs look diffuse, and have no a well-defined shape that can be directly fit into the two-type classification. One possibility is that they are normal CMEs not seen as such due to the projection effects (e.g., Cremades and Bothmer, 2004). Other possibilities should also be explored. For example, they might be due to the complex magnetic topology, which may be original (Roussev et al., 2007; Zhang et al., 2007; Zhukov and Veselovsky, 2007) or due to interchange reconnection with ambient magnetic structures (Attrill et al., 2006; Gibson and Fan, 2008). Further investigation is definitely required.

Based on the fact that narrow CMEs are the extension of EUV jets in the low corona, Wang et al. (1998) proposed that they result from the magnetic reconnection between a small-scale magnetic bipole, e.g., an emerging magnetic flux or a coronal loop, and the unipolar flux, i.e., the open magnetic field in the coronal holes, as depicted by the left panel of Figure 3. Note that such a model is the extension of the reconnection model for SXR jets (Shibata et al., 1992). At the same time, as the result of magnetic reconnection, a compact solar flare appears near the solar surface.

For the normal CMEs, most of them can be considered as an erupting flux rope system, presenting the typical three-part structure, i.e., a convex-outward frontal loop which is immediately followed by a cavity with an embedded core. Note that one or two components might be absent in the white-light images. For instance, the cavity may be missing due to insufficient instrument sensitivity or an unfavorable angle. As depicted by the right panel of Figure 3, the eruption of normal CMEs can be described as follows (see Forbes, 2000, for a review): A flux rope (a twisted or strongly sheared core magnetic field in a more general sense), which may or may not hold a filament/prominence, is kept in equilibrium by the overlying envelope magnetic field lines which are line-tied to the solar surface. The flux rope rises due to some reason, e.g., magnetic rearrangement, the loss of equilibrium, or some instability. Some of the magnetic field lines straddling over the 


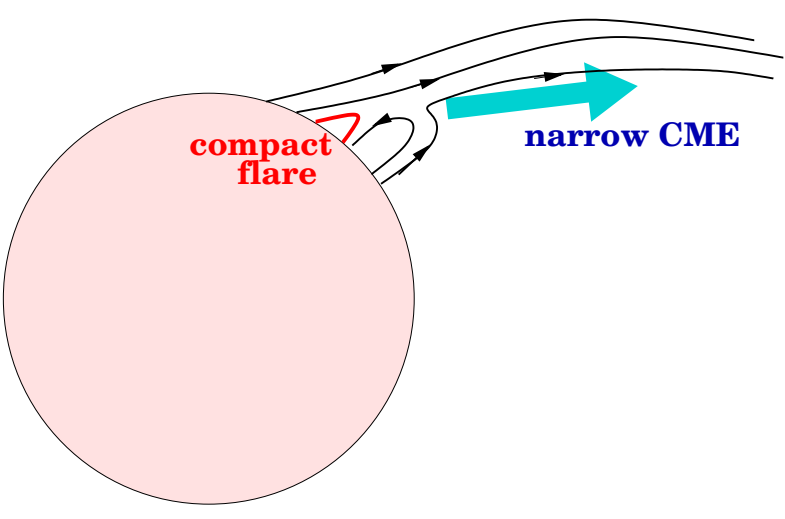

(a)

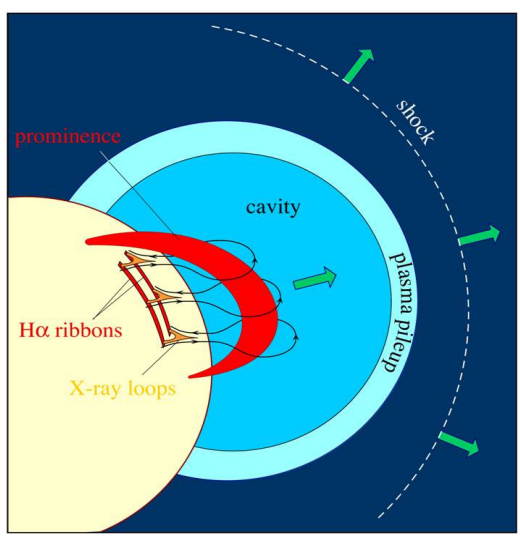

(b)

Figure 3: Schematic models to describe the eruption of narrow CMEs (left) and normal CMEs (right). The right panel is taken from Forbes (2000).

flux rope are stretched up, forming antiparallel magnetic field in the wake of the rising flux rope. In the three-dimensional (3D) case, the upward and the downward magnetic field lines are not strictly antiparallel, with a certain component in the direction along the photospheric magnetic inversion line below the flux rope. As the flux rope rises, the upward and downward magnetic lines below the flux rope approach each other to form a current sheet. Microscopic instabilities of the current sheet enable resistive or collisionless magnetic reconnection. Such a fast reconnection leads to a solar flare, quite often a two-ribbon flare, below the reconnection point. On the other hand, the magnetic reconnection cuts the line-tied magnetic field lines, which removes the constraint for the flux rope and facilitates the rapid eruption of the flux rope (the details of such a process is as follows: the Lorentz force accelerates the reconnection outflows, and the upward outflow pushes the flux rope to move). Note that the toroidal magnetic flux of the flux rope keeps increasing as the reconnection goes on. The erupting flux rope pushes the overlying closed magnetic lines to stretch up, somehow forming a CME frontal loop and a piston-driven shock ahead of it (see Section 4.4 for the discussion on how the frontal loop forms). If fast reconnection is not excited, the flux rope might also have a chance to erupt due to loss of equilibrium or various ideal MHD instabilities (see Section 4.1 for the debate on the role of reconnection in CME eruptions). In this case, no flares or brightenings are visible near the solar surface.

Note that part of the overlying magnetic loops would be stretched up, extending to the interplanetary space along with the flux rope (e.g., Linker et al., 2003). The stretched field lines are not really open field (e.g., Riley, 2007), although they are often called "open field" since the closure takes place far from the low corona. Other parts of the overlying magnetic loops, especially those near the two ends of the flux rope, might slip to the two ends of the flux rope that are anchored to the solar surface (e.g., Low, 1997).

From the above pictures, it is seen that flares and CMEs, when they are associated, can be considered as different manifestations of the same physical process, namely the conversion of magnetic free energy to radiative and kinetic energies, respectively (see Harrison, 1995). Such a model was originally developed to interpret the various faces of solar flares, including the associated plasmoid ejections, by Carmichael (1964), Sturrock (1966), Hirayama (1974) and Kopp and Pneuman (1976) among others (see Švestka and Cliver, 1992, for a review), hence was later called CSHKP model. The model was dubbed "standard model" for CME/flares (Hudson and Cliver, 2001). It

Living Reviews in Solar Physics

http://www. livingreviews.org/lrsp-2011-1 
is stressed that such a standard model is just a phenomenological model based on observations. It describes the overall evolution of CME/flares, with many details being not included. With new multiwavelength observations, such a theoretical framework needs to be improved in order to cover the various phases of the CME eruptions from their birth to their pilgrimage to the interplanetary space. First, we are still not quite sure what is the progenitor that is ready to be triggered to erupt as a CME. The rough picture is described as follows: magnetic field, which is generated at the tachocline layer, emerges throughout the convection zone and the lower atmosphere into the tenuous corona. In response to the continual flux emergence and photospheric motions, the coronal field keeps adjusting to form a more and more complex magnetic structure in a quasi-static way (actually pervasive magnetic reconnection happens, producing a pool of small brightenings). At a certain stage, the magnetic structure reaches a nonequilibrium state or a metastable state that has the potential to release part of its magnetic energy. In this picture, it is still an open question whether the pre-CME structure should always possess a flux rope. Or, the so-called flux rope is actually an extreme case of the ordinary magnetic flux tube with a certain twist. The second issue is how the progenitor is triggered to deviate from the equilibrium state. In this aspect, the statistical investigations of the correlation between CME onsets and other phenomena are of extreme significance. The third issue is how a CME is accelerated. The related questions involve (1) whether magnetic reconnection is a necessary condition; (2) how important the interaction between the ejecta and the solar wind is; (3) the effect of prominence mass drainage, among others. The fourth issue is how the CME is related to the accompanying phenomena, such as solar flares, type II radio bursts, Moreton waves, EIT waves and dimmings, transient coronal holes, etc. The fifth issue is how the CME evolves to an interplanetary CME (ICME) and how the CME properties affect the geomagnetic activity. In the following subsections, we present the current viewpoints on some of the above-mentioned issues (see also van Driel-Gesztelyi and Culhane, 2009).

\subsection{Progenitor}

It is always interesting for researchers to know what kind of structures have the potential to erupt as a CME.

For narrow CMEs, the progenitor is the open magnetic field, usually the coronal hole. When new magnetic flux emerges (or a coronal loop moves to) near or inside the coronal hole, it reconnects with the open field of the coronal hole. The elongated reconnection outflow may appear as a jet-like narrow CME.

For normal CMEs (hereafter, "CMEs" refer to the normal CMEs except otherwise specified), the progenitor should be a strongly twisted or sheared magnetic structure, which has stored a lot of nonpotential energy. On the other hand, the structure should have a potential to erupt while being kept in a metastable equilibrium or being close to a nonequilibrium state, presumably by the line-tying effect at the footpoints and the overlying closed magnetic field. In the $2.5 \mathrm{D}$ case, a simple force-free magnetic arcade with only shear but no twist might be too stable to be ready for eruption (Hood and Priest, 1980). However, in the 3D case, a simply sheared magnetic structure might be eligible for the CME progenitor. The closed magnetic fields on the Sun typically consist of active regions and bipolar magnetic field straddling over quiescent filaments, which are often the source regions for CMEs. Their eruption may take away the overlying large-scale magnetic loops, e.g., the interconnecting loops or transequatorial loops (Khan and Hudson, 2000).

As mentioned before, it has been known that CMEs are strongly associated with eruptive filaments. Since some active region filaments and almost all quiescent filaments are of the inverse polarity type (Leroy et al., 1983; Bommier and Leroy, 1998), which are well described by the flux rope model (Kuperus and Raadu, 1974), it was frequently assumed that the flux rope system is the ideal model for the progenitor of CMEs, i.e., a sheared and twisted field embedded in a less-sheared magnetic system. Considering many CMEs originate from helmet streamers, Low and Hundhausen 
(1995) describe the CME progenitor as a flux rope embedded under a coronal streamer, with a filament being supported at the bottom, as shown by Figure 4. In such a paradigm, the highdensity streamer, i.e., the shaded area, evolves to the frontal loop of the CMEs, the prominence corresponds to the bright core of the CME. In between, there exists a low density cavity, which might be due to stronger magnetic field (Low and Hundhausen, 1995) or the plasma evacuation related to the prominence condensation. Such a 3-part structure was frequently found in streamers before eruption (Gibson et al., 2006b).

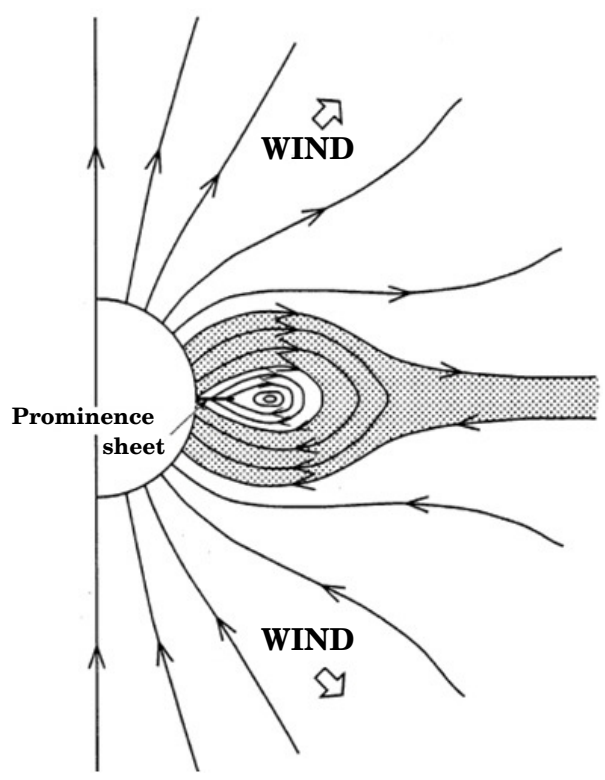

Figure 4: The flux rope model for the CME progenitor, where the shaded area corresponds to the dome of a helmet streamer surrounding a cavity in the middle, and a prominence is located at the bottom of the flux rope. Note that the self-closed flux rope is the projection of a 3D helical flux rope (from Low and Hundhausen, 1995).

From the theoretical point of view, there are several advantages to have a flux rope in the CMEs progenitor as depicted in Figure 4:

(1) The simple but fundamental model for twisted field lines, which carry electric current and therefore magnetic free energy, is a flux rope (Low and Berger, 2003).

(2) The magnetic flux rope system gracefully matches the three-part structure of CMEs, as mentioned above.

(3) As long as the flux rope rises somehow, e.g., due to magnetic rearrangement or a certain instability, a current sheet naturally forms near the separator or a hyperbolic flux tube below the flux rope (Titov et al., 2002). The reconnection in the current sheet leads to the eruption of the flux rope and the overlying magnetic loops, as well as a solar flare near the solar surface, i.e., it fits the classical CSHKP model very well. Even without reconnection, the strongly-twisted flux rope may also erupt due to its intrinsic instabilities (see Section 4.1 for details).

Since the coronal magnetic field cannot be measured directly, the flux rope model can only seek for indirect evidence from observations. Magnetograms showed that the magnetic field is already twisted as it emerges out of the photosphere (Kurokawa, 1987; Lites, 2005). Through Yohkoh satellite observations, Rust and Kumar (1996) found that the geometry of the sigmoidal coronal loops before eruption is in accord with the flux rope model. Although the sigmoidal structure was later confirmed to provide evidence for the flux rope model, their idea, i.e., the sigmoid is just

Living Reviews in Solar Physics

http://www. livingreviews.org/lrsp-2011-1 
the flux rope itself, was later criticized (Gibson et al., 2006a). Canfield et al. (1999) further found that sigmoidal active regions are significantly more likely to be eruptive. Note, however, that some sigmoidal structures consist of many isolated structures, which appear to be a sigmoid or double J-shaped loops due to projection or poor resolution (Glover et al., 2002), and many CMEs are born in active regions without sigmoidal loops. Besides the helical structure, cavity patterns were also observed in the pre-CME structures in SXR (Hudson et al., 1999). In addition, radio imaging observations also showed a depressed region overlying an erupting filament, which was explained as a flux rope by Marqué et al. (2002). However, it should also be kept in mind that a flux rope might not be the only possibility for the pre-CME cavities. Any plasma dilution process, e.g., a slowly stretching loop, may produce the emission depletion.

On the other side, the nonlinear force-free magnetic extrapolation based on the photospheric vector magnetogram on 2000 July 14 shows a strongly twisted flux rope structure embedded in a simple bipolar magnetic arcade, as seen from Figure 5 (Yan et al., 2001). It is noted that the number of the twist is sensitive to the treatment of the $180^{\circ}$ ambiguity of the horizontal magnetogram. For example, with the same vector magnetogram as in Yan et al. (2001), He and Wang (2008) obtained a less-twisted flux rope. With the state-of-the-art extrapolation technique (Schrijver et al., 2008; Guo et al., 2010) and temperature tomography (Tripathi et al., 2009), the existence of a flux rope prior to some eruptions was further confirmed. Before these efforts, the linear force-free extrapolation already showed flux rope structures, with its magnetic dips being in great accordance with the $\mathrm{H} \alpha$ filament structures (Aulanier and Demoulin, 1998). Concaveoutward features behind the CME leading loop as found by Illing and Hundhausen (1983) in some CME events were considered to be consistent with the flux rope model (Chen et al., 1997). Further statistics by Dere et al. (1999) and St Cyr et al. (2000) indicates that $25 \%-50 \%$ of the CMEs observed by the SOHO/LASCO coronagraph contain a helical flux rope. It is probable that many other events also possess similar helical magnetic structures, which did not show up in the white-light images due to low emissions. Some of these helical flux rope structures may exist before the eruption as discussed above. However, it was also argued that some of the helical flux ropes observed by coronagraphs might be formed during the eruption of the CME (Dere et al., 1999; Gosling, 1999; Amari et al., 2003b).

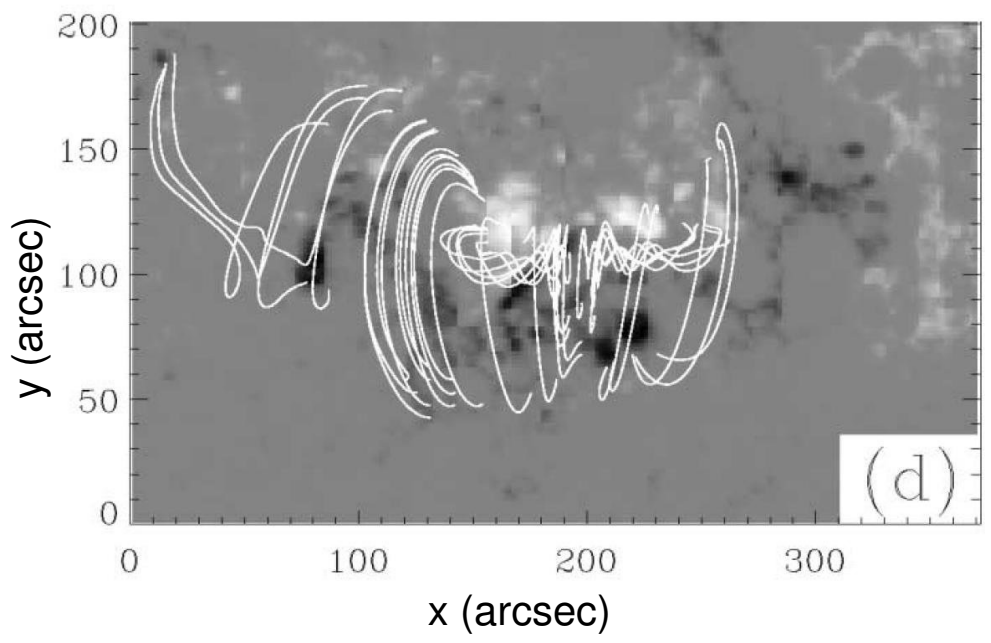

Figure 5: Nonlinear force-free coronal magnetic field lines (white lines) overplotted on the photospheric magnetogram (gray) on 2000 July 14. The horizontal and vertical axes are in unit of arcsec. (from Yan et al., 2001). 
It should be kept in mind that a flux rope is not the only configuration that can carry strong electric current. Strongly sheared field with weak twist can also contain enough magnetic free energy (e.g., Antiochos et al., 1994; Amari et al., 1996). Furthermore, it might be misleading for some literature to illustrate the flux rope model in a two-dimensional (2D) plane. For example, in all 2D models, the flux rope is described to be detached from the solar surface, which means that all the field lines in the flux rope extend to infinity in the Cartesian coordinates (when the third component of the magnetic field is present) or are twisted annules circling the Sun in the spherical coordinates. It was proposed that such a detached flux rope makes the whole system possess more magnetic energy that the open field with the same flux distribution in the photosphere (see the discussion about the Aly-Sturrock constraint in Section 4.1). However, such a detached structure is certainly contradictory with observations. Considering that an observed CME occupies only a part of the solar corona, detached magnetic structure in the 3D space can only be an isolated magnetic island, with magnetic field lines self-closed within a limited volume. It rules out the possibility to form a prominence through chromospheric injection (Priest et al., 1989). It is also hard to believe that such a structure exists and is stable in the corona. A possible stable system might be some detached field lines interwound with other simply connected field lines, although such an analytical solution has not been obtained.

Therefore, a flux rope structure might be like that an arcade of twisted field lines, coming out of the positive polarity in the photosphere, wind one or more turns in the corona making magnetic dips with the inverse polarity, and then go back to the negative polarity in the photosphere (Priest et al., 1989). What remains unknown is the number of the winding. Through either MHD numerical simulations or coronal field extrapolations, some colleagues showed a number larger than 2 (Yan et al., 2001; Roussev et al., 2003), whereas others suggest a number less than 2 (Amari and Luciani, 1999; Aulanier and Demoulin, 1998; Régnier and Amari, 2004; van Ballegooijen, 2004; Su, 2007; Savcheva and van Ballegooijen, 2009) as illustrated in the left panel of Figure 6. In the latter case that a filament is present, the dip of each magnetic field line holds a thread of the filament. More importantly, through MHD simulations, Antiochos et al. (1994) demonstrated that the nonuniform shearing motion can also inject weak twist into the coronal field, leading to an inverse polarity configuration with far less than a half turn. Therefore, a flux rope configuration is even not necessary for the inverse-polarity filaments.

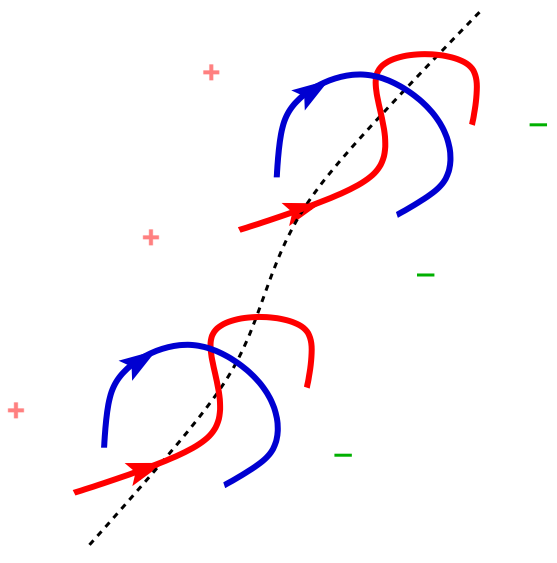

(a)

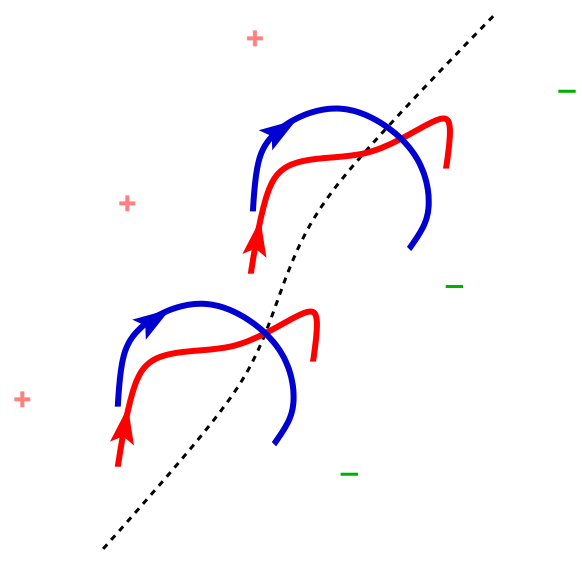

(b)

Figure 6: Schematic sketch of two types of the pre-CME magnetic structures. (a) The core field has an inverse polarity at its dips; (b) the core field has a normal polarity at its center, where there may or may not exist dips. 
It is noticed that many active region filaments, which are often of the normal polarity type (Leroy, 1989), may also erupt to form CMEs. For the normal polarity filaments, the magnetic structure may or may not contain a flux rope, as modeled by Malherbe and Priest (1983), where their Figure 4c corresponds to the magnetic configuration with a flux rope, and their Figure 4b to the configuration without a flux rope. The magnetic configuration in the case with a flux rope has a potential to erupt and can also be fit into the CSHKP model with slight revisions as schematically shown by Low and Zhang (2002, see also Figure 37 in this paper). The magnetic configuration in the case without a flux rope is the classical Kippenhahn and Schlüter (1957) model, which seems difficult to be fit into the CSHKP model. However, we emphasize here that the difficulty also results from the misleading $2 \mathrm{D}$ plot, which can be resolved in the 3D plot as explained in the next paragraph. Considering that the magnetic field in a filament is always directed with a small angle relative to the magnetic inversion line (Leroy et al., 1983), the magnetic configuration of the CME progenitor with a normal polarity filament (in the case without a flux rope) might be an arcade of sheared magnetic loops with the normal polarity dips, which are all confined below less-sheared bipolar loops, as depicted in the right panel of Figure 6.

Therefore, corresponding to two types of filaments, i.e., with inverse and normal polarities, there might be two types of magnetic configurations for the CME progenitors, one is the twisted field model, and the other is just an arcade of sheared arcades as illustrated by Figure 6, which is the 3D extension of the 2D illustration of Figure 3 in Low (1996). It is also possible to have both inverse and normal polarities in one magnetic configuration (Antiochos et al., 1994; Aulanier et al., 2002). It has already been shown that as the twist increases, a sheared field line would form a dip with the normal polarity, and the normal polarity can transit to the inverse polarity either due to further twist or reconnection below the dip (Priest et al., 1989). Note that non-uniform shearing motions contain a twist component (Antiochos et al., 1994). Therefore, neither the magnetic dips nor the polarity type is crucial for the progenitor to be ready for eruption. The crucial point, which is common for both cases shown in Figure 6, is that an arcade of strongly-sheared flux tubes, which can be called core field, is restrained by the overlying less-sheared bipolar magnetic loops, which can be called envelope field following the terminology in Moore and LaBonte (1980). Such a configuration enables the existence of one long bald patch, two bald patches with one separator (Figure 6a, or see Titov and Démoulin, 1999), a hyperbolic flux tube (e.g., Titov et al., 2002), which degrades to a magnetic null point in a 2D case, or a quasi-separatrix layer (Figure 6b) in the core field. The merit for such a configuration to be the CME progenitor is that whenever the strongly-sheared core field rises due to either magnetic rearrangement or instability, the envelope field is stretched up, and the magnetic separator would collapse into a current sheet under the core field, similar to the $2 \mathrm{D}$ case where a magnetic null point can collapse to form a current sheet. The magnetic reconnection of this current sheet gradually removes the magnetic tension force of the overlying field lines, and facilitates the rapid eruption of the core field into the interplanetary space.

Regarding the debate whether the flux rope exists before eruption or is formed during eruption, with the above discussions we come to a conclusion that in some CME/flare events, especially those associated with quiescent filament eruptions, the flux rope may exist before eruption as shown in the left panel of Figure 6, however, for some normal-polarity filament eruptions, the flux rope may be formed during eruption via reconnection, e.g., as the core field lines in the right panel of Figure 6 (red) reconnect with each other.

Regarding the CME progenitor, i.e., the strongly sheared and/or twisted core field restrained by the overlying envelop field, two issues are worthy to be clarified by future MHD numerical simulations:

(1) The helical flux rope in the left panel of Figures 6 was sometimes plotted as twisted field lines winding many times all the way through the whole spine of the associated filament, which would be difficult to explain why only a segment of a long filament corridor erupted in many events. 
The probable situation would be, as mentioned above, that the flux rope consists of an arcade of weakly twisted magnetic field lines as shown in Figure 6, and each bundle of field lines holds an $\mathrm{H} \alpha$ thread of the filament at the magnetic dip.

(2) The SXR sigmoids could be the SXR signature of the CME progenitor, which may correspond to the upper part of the core field, with the filament threads lying on the dips of the lower part of the core field. Generally, the entire core field erupts, which is accompanied by the disappearance of both sigmoids and filaments (Canfield et al., 1999). However, the sigmoid occasionally erupted leaving the filament almost unchanged below the resulting postflare loops (Pevtsov, 2002; Liu et al., 2007). One possibility is that the thick core field experienced a pinch instability in the middle between the upper part (i.e., the SXR sigmoid) and the lower part (i.e., the filament), which led to a current sheet between the sigmoid and the filament. The ensuing reconnection results in the sigmoid erupting upward and flaring loops overlying the filament (see also Gilbert et al., 2000; Gibson and Fan, 2006).

\subsection{Triggering mechanisms}

In the literature, the pre-CME structure, i.e., the CME progenitor, was sometimes called CME precursors, e.g., SXR sigmoids were called CME precursors by Canfield et al. (1999). To avoid any confusion, in this paper we define the progenitor as the unstable or metastable coronal structure that would be the source of a CME, e.g., a sigmoid, and define the precursor as an observable signature associated with the initiation of the CME, i.e., before its main acceleration, which is coincident with the impulsive phase of the associated flare (Zhang et al., 2001a).

As a large-scale eruptive phenomenon, CMEs often reveal thermal or nonthermal signatures during the initiation process just prior to the eruption, i.e., precursors. The CME precursors are very useful since they can be used to predict the occurrence of a CME, as well as to constrain or construct the CME triggering mechanisms (see Gopalswamy et al., 2006, for a review). Before reviewing the triggering mechanisms proposed so far, we have an overview of the possible precursor phenomena that precede CMEs in observations.

\subsubsection{Observational precursors}

The imaging and spectroscopic observations of the CME source region are crucial to find out precursors and to understand how CMEs are initiated. The precursors for CMEs found in the past decades can be summarized as follows:

\section{(1) Helmet streamer swelling and/or slow rise of prominences}

With the Solar Maximum Mission (SMM) satellite observations, Hundhausen (1993) found that most CMEs arise from pre-existing helmet streamers, with the streamers being increasing in brightness and size for days before final eruption. Therefore, the streamer swelling can be used to predict the eruption of CMEs, mainly the limb events. Different from the following precursors, the streamer swelling is the direct imaging of the CME initiation.

A phenomenon intimately linked to the streamer swelling is the slow rise of prominences (Filippov and Koutchmy, 2008).

\section{(2) Reconnection-favored emerging flux}

Feynman and Martin (1995) analyzed 53 quiescent filaments, and found that 17 out of 22 filaments that were associated with emerging bipolar magnetic flux erupted and only 5 out of 31 filaments that were not associated with emerging flux erupted. More importantly, they found that in all cases when the emerging flux was oriented favorably for magnetic reconnection, the filaments were

Living Reviews in Solar Physics

http://www. livingreviews.org/lrsp-2011-1 
observed to erupt. Two examples of new flux emerging inside and outside the filament channel are depicted in Figure 7, which shows the photospheric magnetograms for the two events. Generally, the new flux emergence begins a few days before the filament eruption and typically is still occurring at the time of eruption. It is noted that new magnetic flux emerges all the time and everywhere, especially those weak fields. However, the filament eruption-associated emerging bipolar field has a similar amount of magnetic flux as that of the original filament channel (Feynman and Martin, 1995). Wang and Sheeley Jr (1999) confirmed such a correlation (see also Srivastava et al., 2000; Nitta and Hudson, 2001; Zhang et al., 2001b). Further statistical studies showed that $68 \%$ of the filament eruptions during 1999 to 2003 (Jing et al., 2004) and 91\% of halo CMEs during 1997 to 2005 (Zhang et al., 2008) are associated with magnetic flux emergence. Caution should be taken that these later statistical studies did not consider the polarity orientation and the total flux of the emerging field as done by Feynman and Martin (1995).

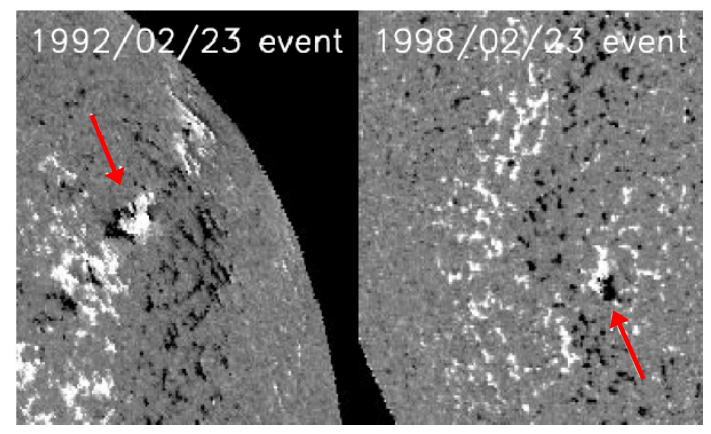

Figure 7: Two examples showing new magnetic flux emerging inside the filament channel (left, the 1992 February 23 event) and outside the filament channel (right, the 1998 February 23 event) (from Chen and Shibata, 2000).

\section{(3) SXR brightenings}

Roy and Tang (1975) studied six flares and found that SXR bursts often accompany the preflare expansion of the filaments. Such a phenomenon was studied by several researchers (Pallavicini et al., 1975; Rust et al., 1975; Webb et al., 1976). As an extension, Simnett and Harrison (1985) studied the relation between the preflare activity and CMEs, and found that there is usually a weak SXR enhancement 15-30 min prior to the linearly extrapolated onset time of the CMEs, which was called SXR precursors for CMEs. As an example, Figure 8 shows an SXR enhancement $\sim 20$ min before the CME-related flare. Caution should be taken that there are many SXR brightenings over the Sun, and high-cadence SXR imaging observations are needed to distinguish whether any pre-CME brightening is spatially and physically related to the CME.

\section{(4) Radio noise storms}

It was found that the radio noise storm continuum emissions, sometimes called type I radio bursts, are associated with the SXR brightenings (Raulin and Klein, 1994; Krucker et al., 1995; Crosby et al., 1996), with the former lasting for a longer time. Evidence was revealed to show that the radio noise storms are related to the initiation of CMEs in a statistical study (Lantos et al., 1981). Ramesh and Sundaram (2001) also found that CMEs often erupted after significant metric noise storm continuum emissions, which are located under the angular span of the associated CMEs. As shown by Figure 9, Wen et al. (2007) found that the noise storm sources are within the CME dimming regions. It is believed that the commencement of the radio noise storm is due 


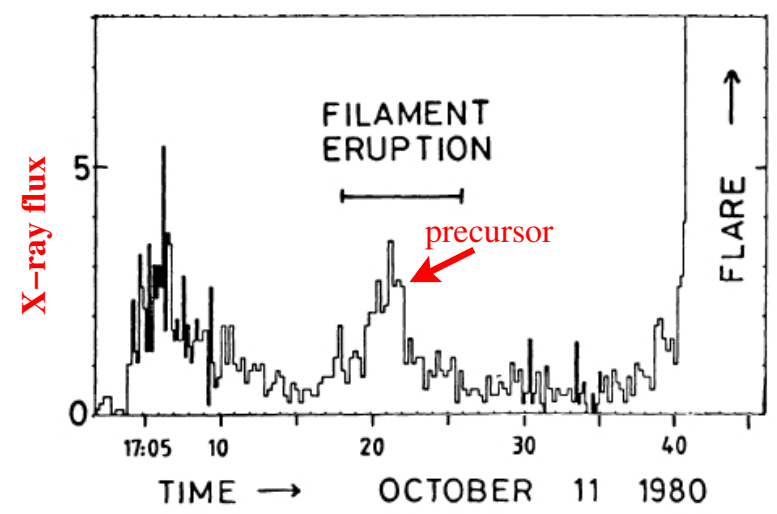

Figure 8: Soft X-ray light curve just prior to a big flare. An SXR enhancement, visible $\sim 20$ min before the main flare, is associated with the eruption of a filament (adapted from Simnett and Harrison, 1985).

to the magnetic restructuring during CME initiations. Waves, e.g., Alfvén waves, induced by the changing magnetic field, slightly accelerate electrons, which then emit radio radiations. On the other hand, the rapid decay of the pre-existing metric radio storms, e.g., above sunspots, might also be related to CMEs (Chertok et al., 2001). However, it is noted that the corona is always in a dynamic state, with radio noise storms happening frequently. Many of them might not be associated with CME initiations.

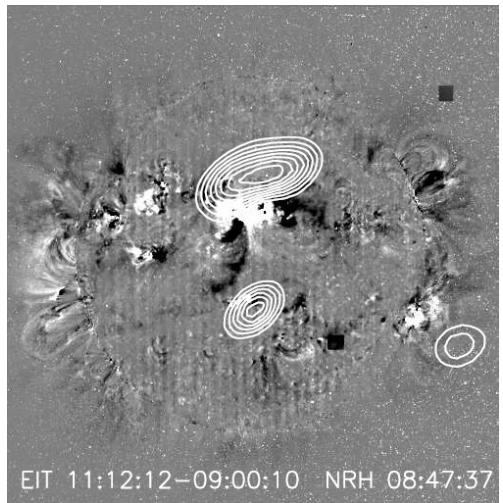

Figure 9: $164 \mathrm{MHz}$ radio noise storm (contour) overplotted on the EIT base difference map (gray) showing the dimming region associated with the CME on 2000 July 14 (from Wen et al., 2007).

\section{(5) Type III radio burst group}

Combining the radio and white-light coronagraph observations, Jackson et al. (1978) found that generally 5-10 hours before the first appearance of a CME in the coronagraph field of view $\left(1.5-6 R_{\odot}\right)$, type III radio bursts appeared intensively, with the occurrence rate being 2.5 times higher than normal, as seen from Figure 10. It is also shown that the faster the CME is, the shorter the interval between the CME appearance and the intense type III radio burst group is. It was revealed that these type III bursts, which are earlier than the main type III burst in the flare impulsive phase, are generally located within $20^{\circ}$ around the central position angle of the CMEs. Jackson et al. (1978), as well as Kundu et al. (1987), proposed that the type III radio burst 


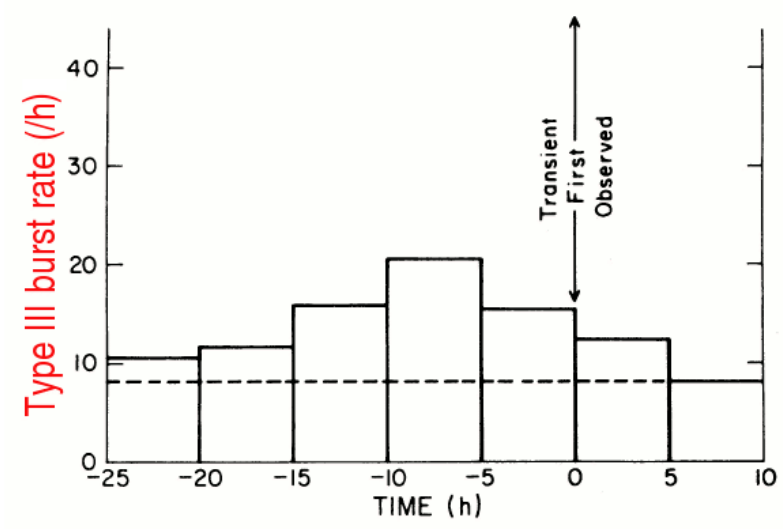

Figure 10: The number of type III radio bursts per hour (histogram) that were observed around the first appearance of the CME (double-ended arrow). Note that the occurrence rate is averaged every 5 hours, and the dashed line marks the average rate of type III bursts over years (from Jackson et al., 1978).

precursor is linked to the CME initiation, resulting from small-scale magnetic reconnection. Klein et al. (1997) clearly showed that the radio burst group-associated magnetic reconnection precedes the ensuing eruption of a large-scale structure.

\section{(6) Filament darkening and widening}

Martin (1980) pointed out that among many preflare precursors, the most typical one is the filament darkening and widening, as illustrated by Figure 11. Generally a flare would occur 1 hour after the appearance of such features (Bruzek, 1951). Since the filament/flare eruptions are the key ingredients of major CMEs, the filament darkening and widening can also be considered as CME precursors.

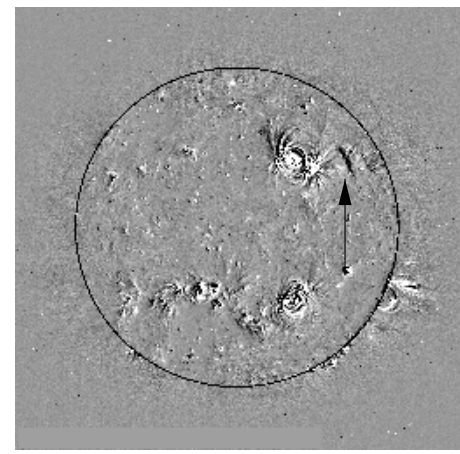

EIT: 09:11-08:54

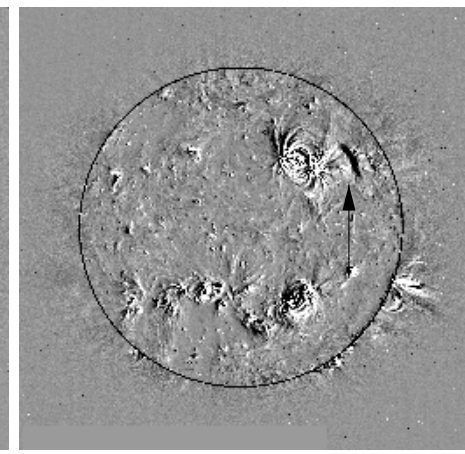

09:25-08:54

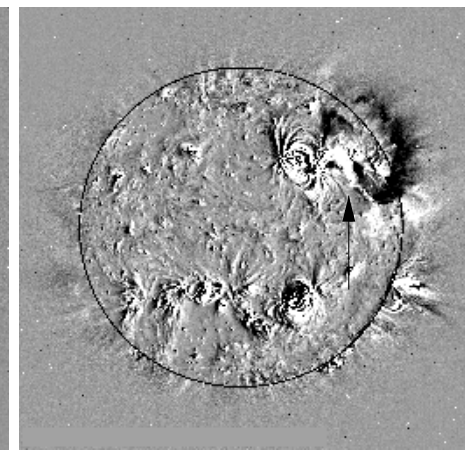

09:47-08:54

Figure 11: EIT base difference images showing the filament darkening and widening before eruption (from Klassen et al., 2002).

\section{(7) Long-term filament/prominence oscillations}

Owing to ubiquitous perturbations in the corona, prominences often oscillate, even in the quiescent state. Generally, the oscillations last $1-3$ times the corresponding period, decaying rapidly. After 
analyzing the spectroscopic data of a prominence eruption event, Chen et al. (2008) found that before the final eruption, the prominence was oscillating for 4 hours, which was almost 12 times the corresponding oscillation period, as shown by the alternating red/blue shifts along the dashed line in the panel (a) of Figure 12. They proposed that this kind of long-term oscillation might be a precursor for a CME, since any triggering process, as a kind of perturbation, can drive the prominence to oscillate. For example, panels (b) and (c) of Figure 12 illustrate how the emerging flux would drive the prominence to oscillate as it triggers the prominence to erupt. They revealed from one event that the oscillating velocity is $\sim 10 \mathrm{~km} \mathrm{~s}^{-1}$, and the spatial displacement is about 2 arcsec, which requires further high-resolution imaging observations to find more events.

(a)

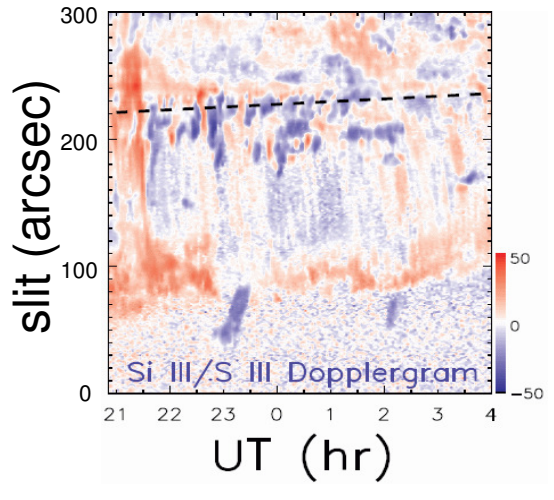

(b)

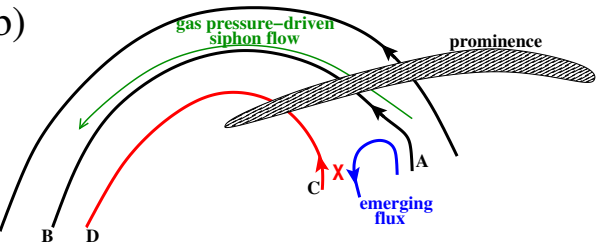

(c)

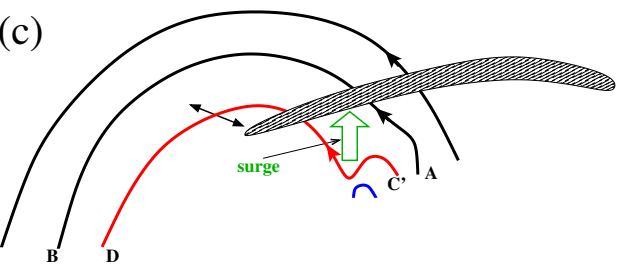

Figure 12: Panel (a): evolution of the Dopplergram along the SUMER slit observed at S III/Si III $1113 \AA$ showing prominence oscillations before eruption, where the Doppler velocity is in unit of $\mathrm{km} \mathrm{s}^{-1}$. Panels $(b-c)$ : new magnetic flux emerges near a filament channel, and reconnects with the pre-existing magnetic field. The localized reconnection drives the prominence oscillation before final eruption (from Chen et al., 2008).

\section{(8) Outward-moving blobs near the edge of streamers}

With the Heliospheric Imagers on board the Solar TErrestrial RElations Observatory (STEREO) satellite, Harrison et al. (2009) identified narrow rays comprised of a series of outward-propagating plasma blobs apparently forming near the edge of the streamer belt prior to many CME eruptions. They suggest that these blobs result from the interchange reconnection between the streamer and the neighboring magnetic field, which removes the downward magnetic tension force holding the streamer static. Therefore, they call this phenomenon as pre-CME fuse.

Among the above-mentioned precursors, only the emerging flux appears well before the onset of CMEs, whereas others are the signatures associated with the initiation of the CME. It should be noted that none of the precursors is a necessary or sufficient condition for CME eruptions. For instance, many CMEs are not triggered by emerging flux (e.g., Démoulin et al., 2002; van DrielGesztelyi et al., 2003). On the other hand, Zhang et al. (2008) pointed out that new magnetic flux frequently emerged in active regions, during which no any eruption happened. Tappin (1991) found that it is also often to have SXR brightenings without any ensuing main flare (nor CME). Therefore, when we try to construct an empirical model for CME forecast, it is important to combine some or all of the above-mentioned precursors together in order to increase the success rate. It is noticed that some of the triggers, e.g., the emerging flux, are only the external cause for the CME eruptions, whereas the magnetic complexity of the CME progenitor is the internal cause.

Living Reviews in Solar Physics

http://www. livingreviews.org/lrsp-2011-1 


\subsubsection{Various triggering mechanisms}

As the magnetic field, which is generated at the base of the convection zone, emerges into the corona, it starts a ceaseless journey. On one hand, new magnetic flux continues to emerge from the subsurface into the corona, colliding with the pre-existing field and producing electric current layers or even current sheets in the highly conducting coronal plasma. On the other hand, photospheric motions with various length scales, e.g., the differential rotation, supergranular, mesogranular, and granular convections, persistently drag the footpoints of all the magnetic field lines to move in both organized and random ways, building up a highly stressed coronal magnetic field (Forbes, 2000). As a result, Poynting flux is continually injected into the corona, and the magnetic energy is thus accumulated gradually. It is generally accepted that the required energy for powering a strong CME comes from the coronal magnetic field, which is in an equilibrium state before it is initiated to erupt. From the mechanical equilibrium point of view, the CME progenitor sustains its equilibrium as the downward magnetic tension force balances the upward magnetic pressure force. Either the decrease of the tension force or the increase of the upward magnetic pressure force would cause the CME progenitor to seek an equilibrium at a higher altitude, and a CME might be then initiated.

Probably in the following two cases the accumulated energy could be suddenly released to host an eruption: (1) the CME progenitor reaches a metastable state (Sturrock et al., 2001); (2) the CME progenitor reaches a state close to the loss of equilibrium or instability (Forbes, 2000). In the first case, the CME progenitor is stable to small perturbations, and a proper trigger with significant observable features is necessary for the eruption, whereas in the second case, any further change of the instability-related parameter in the magnetic field, e.g., the magnetic twist, would directly trigger the eruption.

In the past decades, several triggering mechanisms have been proposed either conceptually or through MHD analysis and/or simulations, as described as follows. However, caveats should be taken that at the present stage, photospheric motions in models and in reality still do not match, and only observational data-driven MHD simulations can provide a quantitative understanding of the eruptive processes involved in CMEs, which is actually under development (Wu et al., 2006). It will also be seen that some of the following models are almost the same in nature, with their emphasis on different aspects of the same triggering process.

\section{(1) Tether-cutting or flux cancellation mechanism}

Moore and LaBonte (1980) analyzed the filament eruption event on 1973 July 29, and found that:

(a) the magnetic field is strongly sheared near the magnetic neutral line;

(b) the filament eruption and the two-ribbon flare were preceded by precursor activities in the form of small $\mathrm{H} \alpha$ brightenings and mass motion along the neutral line;

(c) $\mathrm{H} \alpha$ precursor brightening and the initial brightening of the flare are both located in the vicinity of the steepest magnetic field gradient.

Piecing these features together, they proposed the tether-cutting mechanism: as depicted by Figure 13, a filament is supported somehow, e.g., on dips, by magnetic field (not plotted in the figure) that is nearly aligned with the magnetic inversion line (dashed). Just around the filament, the magnetic field is still strongly sheared, e.g., the field lines $\mathrm{AB}$ and $\mathrm{CD}$, which probably correspond to the associated SXR sigmoid. These strongly-sheared core field is overlaid by less-sheared envelope magnetic arcade. Before the filament erupts, all the field lines around the filament, except those holding the filament threads, are almost in a force-free state, i.e., the downward magnetic tension force is balanced by an outward magnetic pressure force. As the magnetic shear increases, the negative leg of the field line $\mathrm{AB}$ is close to be antiparallel to the positive leg of the field line $\mathrm{CD}$, forming a strong current sheet in between (left panel of Figure 13). Owing to micro-scale instability-driven anomalous resistivity or many other non-ideal effects (such as the Hall effect, 


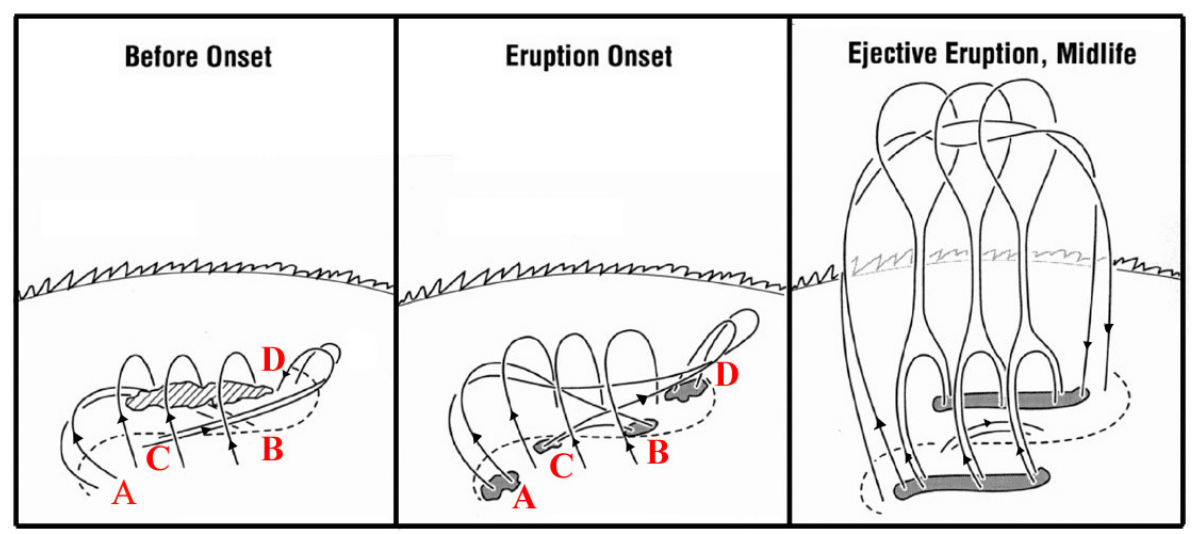

Figure 13: The tether-cutting triggering mechanism for CMEs. Left: strongly sheared core field is restrained by the overlying less-sheared envelope field; Middle: The reconnection between field lines AB and CD triggers the core field to rise; Right: The rising core field stretches up the envelope field, forming a current sheet below the core field (adapted from Moore et al., 2001).

electron inertia, and so on), magnetic reconnection commences. As a result of the reconnection, the line-tied field lines $\mathrm{AB}$ and $\mathrm{CD}$, which are like tethers constraining the filament, are cut from being tied to the photosphere, forming a long field line AD and a short loop CB (middle panel). Such a reconnection would also produce the observed $\mathrm{H} \alpha$ brightenings and mass motions along the magnetic inversion line. Following the reconnection outflow, the long loop AD expands upward, and the small loop CB shrinks down or even submerges down (Wang, 2006).

This process can be easily understood, since after reconnection, the loop AD is concave-upward near the reconnection site, which imposes an upward magnetic tension force on the loop, and the short loop CB is concave-downward, which imposes a downward tension force on the loop. As the localized reconnection goes on, the core field near AD pulls up the filament to rise, by which all the overlying envelope magnetic field are stretched up, forming an elongated current sheet above the magnetic neutral line (right panel of Figure 13). Such a tether-cutting process until now (from the left to the middle panels of Figure 13) can be regarded as the triggering phase of the whole CME eruption. Such a process results in the magnetic configuration required in the CSHKP model, and the magnetic reconnection of the newly-formed current sheet speeds up the filament eruption to form a CME, and produces a two-ribbon flare near the solar surface at the same time.

A similar mechanism was proposed by van Ballegooijen and Martens (1989), who pointed out that the magnetic flux cancellation near the neutral line of a sheared magnetic arcade would produce helical magnetic field lines, i.e., a flux rope, that can support a filament, and that further cancellation can result in the eruption of the previously-formed filament, as depicted by Figure 14 and numerically simulated by Amari et al. (2003b). The flux cancellation model is the same as the tether-cutting model in nature. The difference, if any, is that the flux cancellation model might emphasize a more gradual evolution of magnetic reconnection in the photosphere, while tether-cutting is a relatively more impulsive process occurring in the low corona. Besides, the flux cancellation also allows the flux rope to grow and its axis to rise. A current sheet might be formed as the flux rope rises, creating the required magnetic configuration for the CSHKP model. The flux cancellation would also increase the twist of the core field, which might also drive an ideal MHD eruption (see Section 4.1 for the discussions about ideal MHD eruptions).

The tether-cutting model, however, does not tell how the strongly sheared core field is formed. From the observational point of view, shearing motions may be one effective way to increase the magnetic shear near the polarity inversion line (PIL). Therefore, in the next item, we describe a

Living Reviews in Solar Physics

http://www. livingreviews.org/lrsp-2011-1 

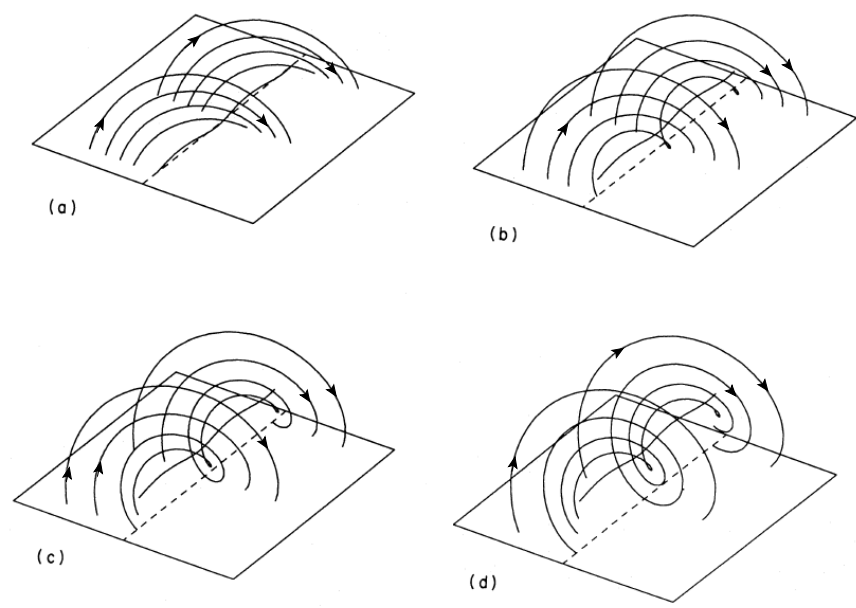

Figure 14: Flux cancellation in a strongly sheared magnetic arcade leading to the formation and levitation of a flux rope. Further cancellation leads to the eruption of the flux rope (from van Ballegooijen and Martens, 1989).

similar model, i.e., the CME initiation by localized shearing motions.

\section{(2) Shearing motions}

The shearing motion is indeed one important way for the corona to build up free energy (Low, 1977). A lot of observations also revealed that many CME source regions experienced strong shearing motions before eruption (e.g., Deng et al., 2001). Therefore, it is not surprising that the shearing motion was proposed to be a trigger mechanism for CMEs (e.g., Aly, 1990). The shearing motion localized near the PIL would produce a strongly-sheared core field, which is covered by a less-sheared envelope field. Thus, this model well explains the required magnetic configuration in the tether-cutting model. In this sense, these two mechanisms are the same in nature.

As illustrated by Barnes and Sturrock (1972), the coronal arcades inflate as the shearing motion is imposed at the bottom boundary. It was conjectured by Klimchuk (1990) that the shearing of simple arcade configurations always results in an inflation of the entire field. The 2.5-dimensional MHD simulations performed by Mikić and Linker (1994) in the spherical coordinates indicate that subject to the localized shearing motion, the magnetic field expands outward in a process that stretches the field lines and produces a tangential discontinuity, i.e., a strong current sheet, above the PIL. Therefore, the shearing motions can be regarded as a triggering mechanism since it paves the way for the potential eruption of the arcade. Once resistivity is excited in the current sheet, magnetic reconnection sets in, and leads to an impulsive release of magnetic energy along with the ejection of a plasmoid. The effects of the shearing profile and background solar wind models on the triggering were numerically studied by Jacobs et al. (2006) with 2.5D MHD simulations in the axisymmetric coordinates. It is shown that (1) a faster shearing velocity leads to a faster eruption; (2) a wider shearing region facilitates the formation of a flux rope; (3) the evolution is dependent on the chosen solar wind model.

It is important to note that some of the plasma shearing motions in observations might not the the driver of the magnetic shear, they might be the result of magnetic flux emergence, as found in MHD simulations (Manchester IV et al., 2004).

Different from the above numerical models where the magnetic shear increases monotonically, Kusano et al. (2004) found that the injection of the reversal magnetic shear at the bottom boundary can also trigger the CME eruption, via a series of two different kinds of magnetic reconnection. 
It is noted that, in most numerical simulations the evolution of the photospheric field is too fast compared to the very gradual accumulation of the magnetic energy in the solar atmosphere. This is true for the simulations mentioned above, as well as below.

\section{(3) Magnetic breakout model}

In order to circumvent the Aly-Sturrock constraint (Aly, 1991; Sturrock, 1991, see discussions in Section 4.1), Antiochos et al. (1999) proposed the so-called magnetic breakout model, as shown in Figure 15. The essence is that the initial magnetic configuration consists of a quadrupolar topology, with a null point being above the central flux system. As the central flux system experiences shear motions, it expands upward, pressing the X-type null point to form a current layer. If gas pressure and resistivity are ignored, the current layer collapses to be an infinitely thin current sheet, which hinders the upward motion of the central flux system; If gas pressure and resistivity are considered, the current sheet would undergo magnetic reconnection owing to anomalous resistivity or other non-ideal effects. Such a reconnection process removes the constraint of the higher magnetic loops, triggering the eruption of the core field (thick lines). They demonstrated that the final state with the central flux system being fully open possesses a total magnetic energy less than that of the initial state, suggesting that partial opening of a closed magnetic configuration is energetically feasible.

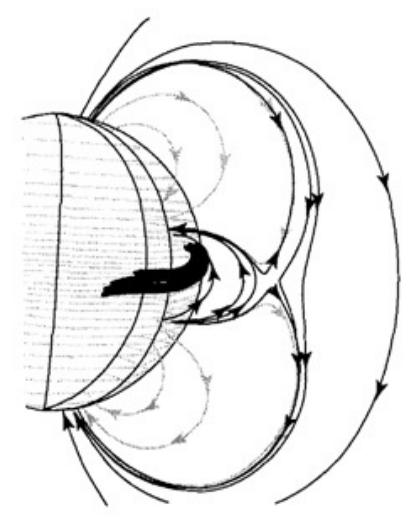

(a)

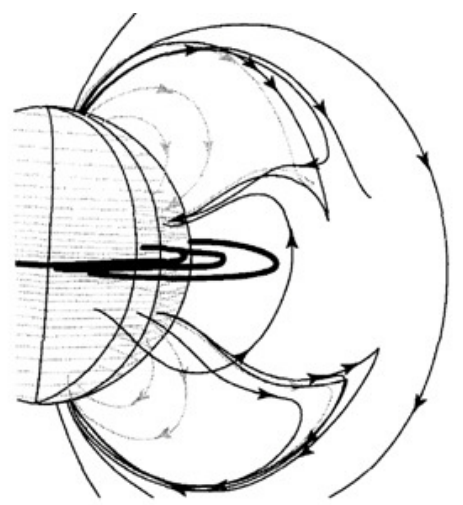

(b)

Figure 15: The evolution of the magnetic field in the breakout model, showing the reconnection above the central flux system removes the constraint over the core field (thick lines), and results in the final eruption (adapted from Antiochos et al., 1999).

As the central flux system rises, a current sheet forms underneath, whose reconnection leads to the drastic formation and eruption of a flux rope. The MHD simulation results of such an eruption process were compared with the CME features in observations (Lynch et al., 2004). Many other numerical models can be classified to this magnetic breakout model, e.g., Archontis and Török (2008) and MacTaggart and Hood (2009).

Note that the essence of this model is that part of the central flux system reconnects with the overlying background magnetic field, by which the constraint over the sheared core field is removed gradually like an onion-peeling process. Such a model can be regarded to be physically the same as the tether-cutting mechanism, and it can be considered as the external tether cutting. It is expected to have SXR bright loops on both sides of the sheared core field and reverse type III radio bursts that are produced by the reconnection-accelerated electrons which, however, might be too weak to be observed. Many authors compared the observed CME initiations with the breakout model 
(e.g., Sterling and Moore, 2004). The first evidence supporting the breakout model was presented by Aulanier et al. (2000), who found a null point above the source region in the extrapolated coronal magnetic field. Another indirect evidence supporting the quadrupolar configuration in the magnetic breakout model is that many quiescent prominences are located on the magnetic neutral lines between bipolar regions (Tang, 1987). From statistical point of view, Li and Luhmann (2006) conducted a statistical study, showing that the CME source regions with quadrupolar background magnetic field, which is crucial for the breakout model, are 3 times less than those with bipolar field. Similarly, Ugarte-Urra et al. (2007) found that the initiation of 7 out of 26 CME events in their study can be interpreted in terms of the breakout model.

\section{(4) Emerging flux triggering mechanism}

By checking the variations of the magnetograms before CME eruptions, Feynman and Martin (1995) found that many CMEs are preceded by emerging flux that possesses polarity orientation favorable for magnetic reconnection between the emerging flux and the pre-existing coronal field either inside or outside the filament channel. Motivated by such a correlation, Chen and Shibata (2000) proposed an emerging flux triggering mechanism for CMEs, as illustrated by Figure 16: when the reconnection-favorable emerging flux appears inside the filament channel as seen in panel (a), it cancels the small magnetic loops near the PIL below the flux rope. Thereby, the magnetic pressure decreases locally. Plasmas on both sides of the PIL, which are initially in equilibrium, are driven to move convergently along with the frozen-in anti-parallel magnetic field under the pressure gradient. As a result, a current sheet forms below the flux rope, and the flux rope is also triggered to move upward slightly. When the reconnection-favorable emerging flux appears outside the filament channel (say, on the right side, as illustrated by panel b), it reconnects with the large-scale magnetic loops that cover the flux rope. The right leg of the large-scale magnetic loop, which is initially rooted very close to the PIL, is re-connected to the right side of the emerging flux, which becomes further from the PIL. The magnetic tension force from the kinked field line pulls up the magnetic loop (the larger thick black line) to move upward, with the flux rope following immediately. As the plasma is evacuated below the flux rope, the X-type null point collapses to form a current sheet.

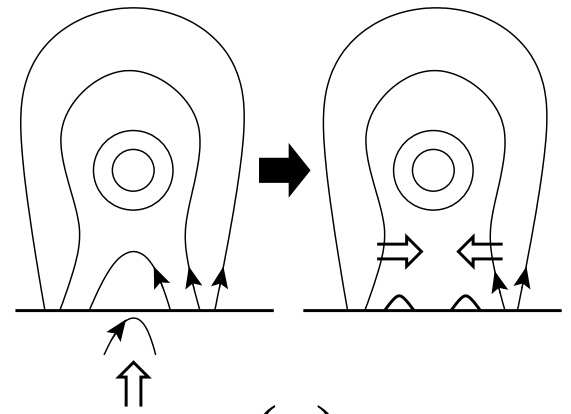

(a)

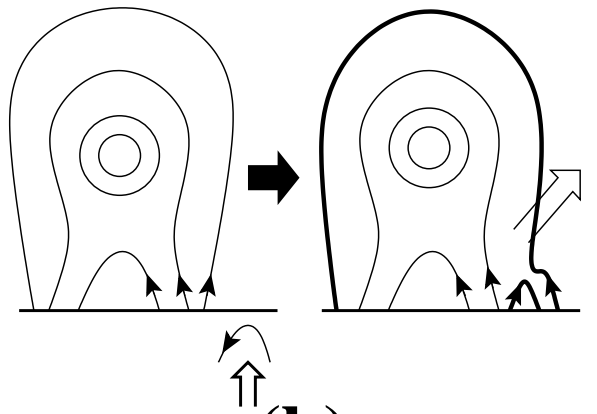

(b)

Figure 16: Schematic diagram of the emerging flux triggering mechanism for CMEs. (a) Emerging flux inside the filament channel cancels the pre-existing loops, which results in the in-situ decrease of the magnetic pressure. Lateral magnetized plasmas are driven convergently to form a current sheet; (b) Emerging flux outside the filament channel reconnects with the large coronal loop, which results in the expansion of the loop. The underlying flux rope then rises and a current sheet forms near the magnetic null point (from Chen, 2008).

In both cases, the core field, i.e., the flux rope here, is initiated to move up, with a current 
sheet forming below, by which a magnetic configuration required for the CSHKP model is formed. The later evolution can well fit into the CSHKP model, i.e., on one hand, the reconnection at the current sheet leads to the formation of a cusp-shaped two-ribbon flare below; on the other hand, the Lorentz force along the upward reconnection jet accelerates the jet and the flux rope system above, as seen from Figures 17 and 18, which show the evolutions of the magnetic field (lines), temperature (color), and velocity (arrows). Note that even though the flux rope in the initial conditions of their simulations is located slightly above the critical height for the torus instability, where $\partial \ln B / \partial \ln y=2.06$, the flux rope was found to be ideally stable without the flux emergence. This implies the difference between MHD evolutions and the circuit analysis of the torus instability. With the plasma $\beta$ chosen in Chen and Shibata (2000), the flux rope erupts with a velocity of $\sim 400 \mathrm{~km} \mathrm{~s}^{-1}$. If the plasma $\beta$ is reduced, the eruption becomes faster.
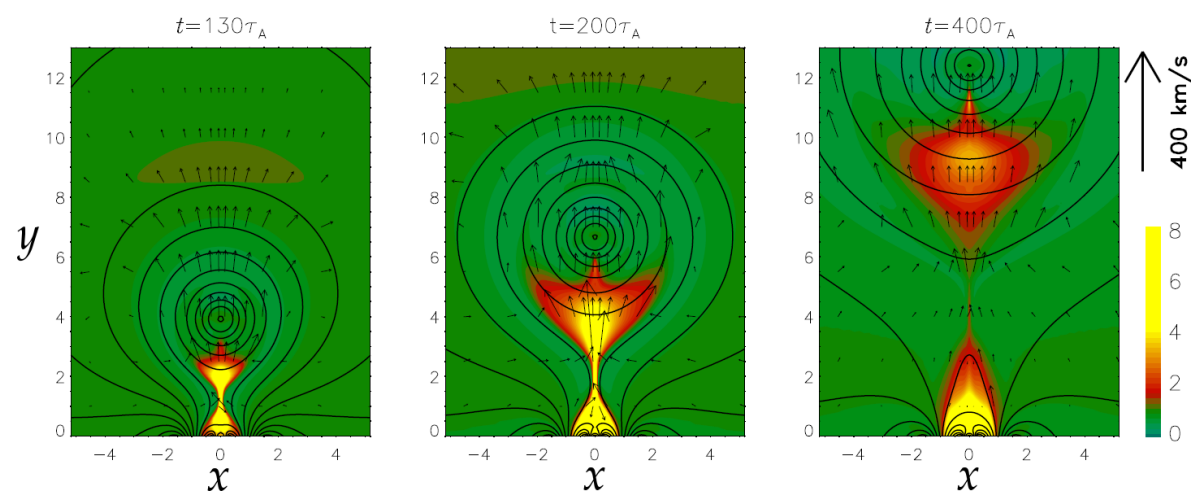

Figure 17: Still from a movie showing Evolutions of the magnetic field (lines), temperature (color, in unit of $10^{6} \mathrm{~K}$ ), and velocity (arrows) in the corona after the flux rope system is triggered to rise when the new flux emerges inside the filament channel (from Chen and Shibata, 2000). The scale for the velocity is shown at the upper-right corner. (To watch the movie, please go to the online version of this review article at http://www.livingreviews.org/lrsp-2011-1.)
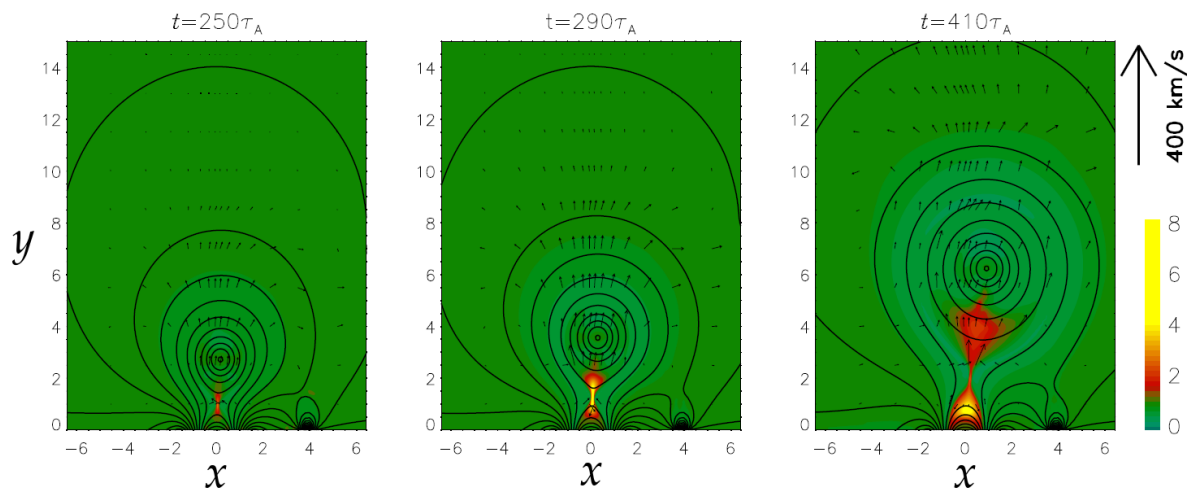

Figure 18: Still from a movie showing Evolutions of the magnetic field (lines), temperature (color, in unit of $10^{6} \mathrm{~K}$ ), and velocity (arrows) in the corona after the flux rope system is triggered to rise when the new flux emerges outside the filament channel (from Chen and Shibata, 2000). (To watch the movie, please go to the online version of this review article at http://www.livingreviews.org/lrsp-2011-1.)

The scale for the velocity is shown at the upper-right corner.

In this model, the onset of the CME is triggered by the localized reconnection between the 
emerging magnetic flux and the pre-existing coronal field. Such a reconnection, which was originally proposed by Heyvaerts et al. (1977) to explain impulsive solar flares, would produce SXR bright points/jets (and $\mathrm{H} \alpha$ surges), which correspond to the SXR precursors well before the main flare as mentioned by Simnett and Harrison (1985). Recent observations indicate that such a localized reconnection would also drive the filament and surrounding magnetic loop to oscillate, which in turn modulates the localized reconnection to be intermittent (Chen et al., 2008). Therefore, it will be interesting to investigate how the CME precursors (2)-(5), and (7) mentioned in Section 3.4.1 can be understood along with the emerging flux trigger mechanism. What can be speculated is as follows: As the reconnection-favorable magnetic flux emerges, it reconnects with the coronal field. The coronal magnetic field is rearranged, and the filament loses its equilibrium. The coronal magnetic loops and the filament oscillate due to the kinked field lines, which may in turn modulate the magnetic reconnection to proceed in a repetitive way (Nakariakov et al., 2006; McLaughlin et al., 2009). The magnetic rearrangement would induce MHD waves, which then slightly accelerate electrons to emit radio noise storms. The repetitive localized reconnection generates SXR brightenings, and accelerates nonthermal electrons, producing a series of type III radio bursts when the background magnetic field happens to be open. A noticeable feature of the localized reconnection in the triggering phase of the CME is that the magnetic reconnection progresses in a repetitive manner, rather than continually.

A similar model was investigated by Archontis and Hood (2008), who simulated the emergence of two twisted flux tubes. They found that the interaction and reconnection between the magnetic fields of the two tubes lead to multiple formation and eruption of flux ropes.

As mentioned in Section 3.4.1, many CMEs were found to be associated with emerging flux. However, it should be kept in mind that some of these CMEs might be triggered by other mechanisms rather than the emerging flux, and the association might be just a coincidence. A parameter survey of this model was conducted by Xu et al. (2008), who showed that whether a new emerging flux can trigger a CME depends on several parameters of the emerging flux: its polarity orientation, its total flux, and its distance to the PIL. Image synthesis was composed by Shiota et al. (2005) in order to compare with Yohkoh SXR images, which indicates that the Y-shaped ejecting structure observed in some CME/giant arcade events corresponds to slow and fast shocks associated with magnetic reconnection. Such a model was also extended from 2D Cartesian coordinates to 3D spherical coordinates (Dubey et al., 2006).

\section{(5) Flux injection triggering mechanism}

Noticing that a curved current loop has a "toroidal force", Chen (1989) extended the previously studied stability analysis of a current loop into the nonlinear phase in order to study its dynamic evolution. As shown in the left panel of Figure 19, the line-tied current-carrying loop, e.g., a flux rope holding a prominence at its bottom, is characterized by the major radius $R$, the minor radius $a$, a toroidal current $I_{t}$, and $\epsilon$ describing how the flux rope closes below the photosphere. With some simplifications, Chen (1989) derived the total force exerted on a toroidal section of the flux rope. According to the Newton's second law, and the plasma in the section is approximated as a particle, the motion of the plasma section is then established. It is found that with some parameters, the flux rope is unstable to radial perturbations, and may expand subsonically for extended periods of time; with some other parameters, the flux rope can expand and then reach a new equilibrium. He also found that if the flux rope carries a relatively large current, a fast eruption can be formed. For example, an initial loop with $\sim 20 \mathrm{G}$ expands at $\sim 1200 \mathrm{~km} \mathrm{~s}^{-1}$, releasing magnetic energy of the order of $10^{32}$ ergs in tens of minutes.

Considering that a prominence is generally stable for weeks before its eruption, Chen (1996) proposed that an initial stable flux rope, as new poloidal flux is injected somehow, is accelerated and becomes eruptive. As an example, the right panel of Figure 19 shows the early evolution of 

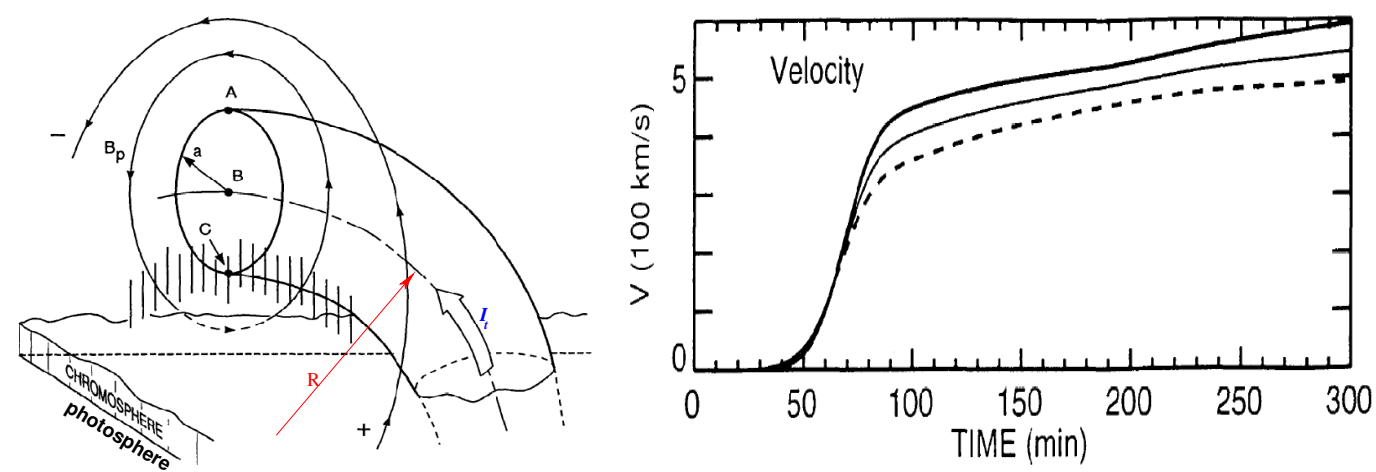

Figure 19: (a) Schematic drawing of the flux injection triggering mechanism for CMEs, where $B_{p}$ denotes the poloidal magnetic field of a flux rope; A, B, and C mark the apex, the centroid, and the bottom of the flux rope, respectively. A prominence is supposed to sit at the bottom of the flux rope; (b) Time evolution of the velocity of the flux rope apex as a certain amount of poloidal flux is injected into the rope (adapted from Chen, 1996).

the velocity of the flux rope in one case. It is noted that the energy release in this model mainly comes from the injected energy. In this sense, this model is different from other storage-and-release models discussed in this subsection.

Such a flux injecting and triggering processes have not been testified by MHD simulations, which should be quite different from the approximated particle dynamics. The flux injection triggering mechanism was sometimes criticized in the sense that the flux injection process would induce too large surface motions that have not been observed (e.g., Forbes, 2000; Schuck, 2010). However, it is interesting to see that an instability exists in the flux rope due to the "toroidal force", as indicated by the equilibrium analysis of the system (Chen, 1989), which might serve as a trigger mechanism. This was called "torus instability", inherited from laboratory plasmas (Kliem and Török, 2006, see Item 6 below).

\section{(6) Instability and catastrophe-related triggering mechanisms}

Since the time scale of the coronal magnetic energy accumulation is very long, whereas flare and filament eruptions commence in a very short time scale, Gold and Hoyle (1960), even early in the 1960 s, proposed that the trigger of the energy release should be related to some kind of instability. Along this line of thought, various instability/catastrophe mechanisms have been developed (see Forbes, 2000, for a review). The basic idea is: as the coronal magnetic field, subject to the photospheric motions and flux emergence, keeps evolving in a quasi-statical way (both ideally and non-ideally), it might reach a critical stage where the equilibrium is unstable (i.e., instability) or no nearby equilibrium state exists anymore (i.e., loss of equilibrium). The pre-CME structure is then at an unstable equilibrium and starts to erupt in the corona where perturbations are ubiquitous.

(a) Kink instability: Sakurai (1976) numerically analyzed the development of the kink instability of a twisted flux tube, as revealed by Figure 20. He pointed out that the kink instability can explain the observed height-time profile of an erupting filament. Hood and Priest (1979) further considered the line-tying effect of the photosphere on the kink instability of the twisted flux tubes, and found that there is a critical twist, above which the flux tube is unstable. In their analysis, the critical twist ranges from $2 \pi$ to $6 \pi$ (see also Mikić et al., 1990). However, these authors did not consider the effect of the external magnetic field overlying the twisted flux tube, which would stabilize the flux tube. Such an effect was studied in the MHD numerical simulations of Török and Kliem (2005) and Inoue and Kusano (2006). According to Török and Kliem (2005), if the

Living Reviews in Solar Physics

http://www. livingreviews.org/lrsp-2011-1 
overlying magnetic arcade decays gently with height, the kink instability would be suppressed after initial development, producing the so-called "failed eruption" found by Ji et al. (2003), and if the overlying magnetic field decays rapidly, the kink instability would lead to an ejective CME. According to Inoue and Kusano (2006), the short axis of the flux rope would also suppress the kink instability. Besides, as pointed by Baty (2001), the classical critical twist for the onset of the kink instability is applicable only for flux ropes with the radius much larger than the axis pitch length. For a general case, it is the local pitch angle that determines the instability.

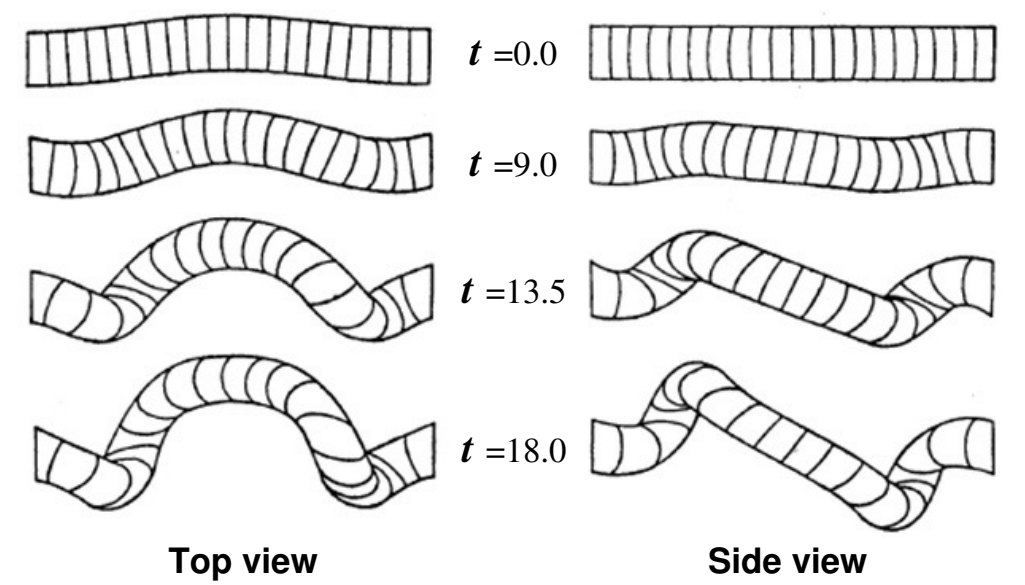

Figure 20: The evolution of the kink instability of a twisted flux tube based on an analytical solution (from Sakurai, 1976).

(b) Torus instability: A current ring is unstable against expansion if the external potential field decays sufficiently fast, e.g., $\partial \ln B / \partial \ln r>3 / 2$ (Bateman, 1978), which was extended to study the dynamics of the flux rope in CMEs (Chen, 1989), and was called torus instability by Kliem and Török (2006), though these early works are based on circuit models, rather than solving full MHD equations.

With 3D MHD simulations, Fan and Gibson (2007) studied the emergence of a flux rope from the subsurface into the magnetized corona. As illustrated by Figure 21, when the background magnetic field declines slowly with height, a strongly-twisted flux tube emerging out of the solar surface may rupture through the arcade field via kink instability (top panels); whereas when the background magnetic field declines rapidly with height, a weakly-twisted flux tube, whose twist is below the threshold for kink instability, still erupts with little writhing like a planary outward expansion. They interpret the latter case as the "torus instability".

It is pointed out here that in their simulations the flux rope is transported kinematically into the simulation box by changing the boundary conditions. Besides, reconnection due to numerical resistivity exists, which would have affected the dynamics of the system, and makes it not straightforward to distinguish ideal MHD instability from resistive instability. The observational result that the extrapolated magnetic field for the torus instability eruption events does not show a systematic rapid decay with height, as presented by Liu (2008), suggests that more factors should be considered.

It is noted in passing that, in several simulations works (e.g., Amari et al., 2004; Manchester IV et al., 2004; Fan and Gibson, 2007; Archontis and Hood, 2008), the flux rope erupts soon after it emerges out of the solar surface. This is slightly different from other "storage-and-release" models, and might explain the CMEs associated with the emergence of a new active region.

(c) Catastrophe: As demonstrated by MHD numerical simulations (e.g., Török and Kliem, 2005), the often used linear instability analysis has the limitation of saying nothing about the 

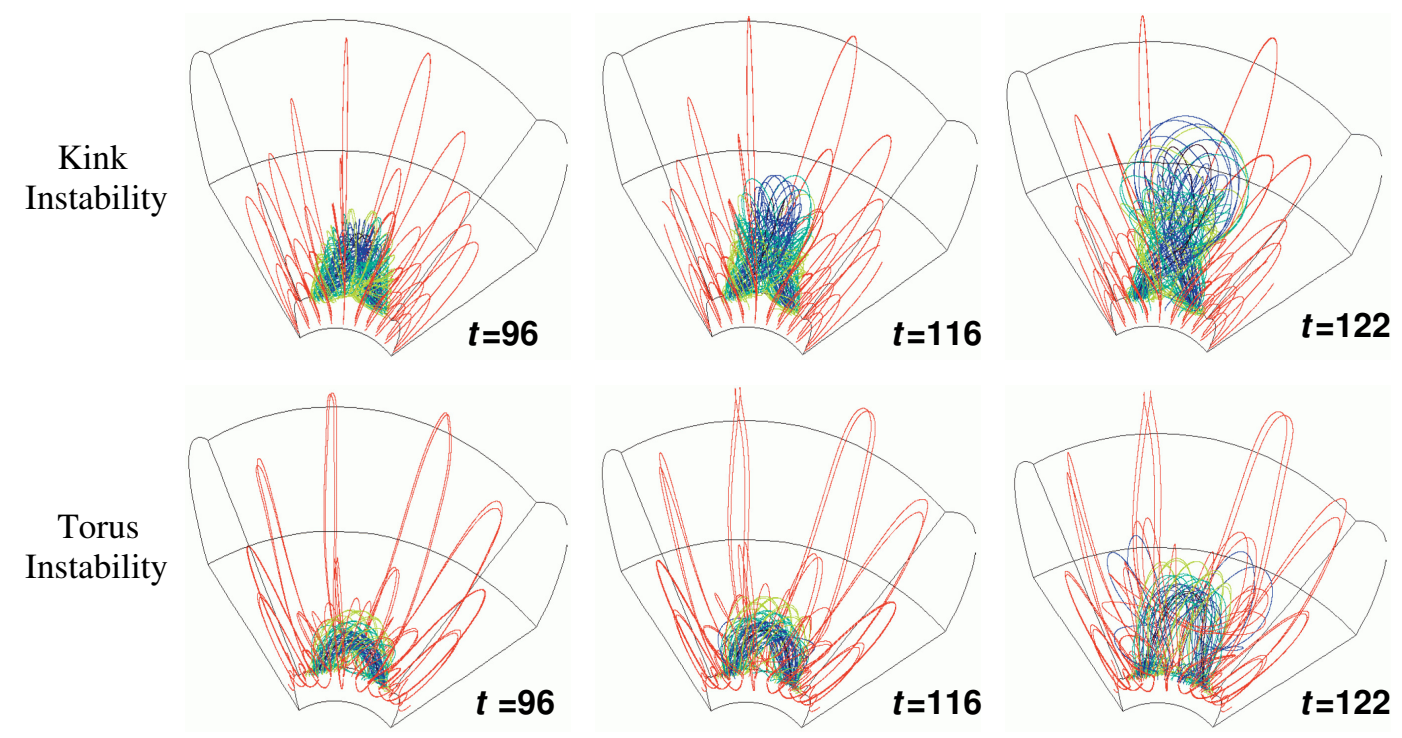

Figure 21: Top: the MHD simulation of the kink instability of a strongly-twisted flux tube emerging from the subsurface to the corona, where the pre-existing magnetic field declines slowly with height. Bottom: the MHD simulation of the torus instability of a weakly-twisted flux tube emerging into the corona, where the pre-existing magnetic field declines rapidly with height (adapted from Fan and Gibson, 2007).

nonlinear development (Priest, 2007). One of the earliest efforts was to seek for the possibility of catastrophe of the magnetic system. Following van Tend and Kuperus (1978), Priest and Forbes (1990) constructed a model to study the equilibrium of a line current filament in the background coronal magnetic field. It was found that as the filament current or twist increases to a critical value, catastrophe takes place. In the ensuing researches on the catastrophic solutions, the measurable quantities, in place of the electric current in the filament, were adopted as the changing parameter. These models can further be divided into two branches, depending whether the triggering process is a resistive or an ideal process, as described separately in the following paragraphs.

As reconnection proceeds below the flux rope in the aforementioned tether-cutting reconnection or flux cancellation model, the flux rope would definitely rise. However, it does not mean that an eruption can be triggered. For example, with zero- $\beta$ MHD simulations, Aulanier et al. (2010) stated that the tether-cutting reconnection below the flux rope does not trigger the eruption of the flux rope, it just pushes the flux rope to rise. It is the torus instability that triggers the onset of the eruption. Alternatively, the 2D analytical solution by Forbes and Isenberg (1991) indicates that as flux cancellation continues near the magnetic neutral line, the flux rope embedded in a bipolar field initially rises smoothly (see Figure 22a-d). However, at a critical point, the flux rope presents a catastrophic behavior, i.e., the flux rope has the potential to jump from a lower altitude (panel e) to a higher altitude (panel f) from the energy point of view, with the flux distribution at the surface unchanged. The energy release in this transition is trivial (the fraction of the magnetic energy released in the transition depends on the background magnetic field, as shown by Forbes et al., 1994), so it might be insufficient to power an eruption. However, the higher state contains a current sheet, whose reconnection would result in the rapid eruption of the filament. Therefore, the flux cancellation-induced transition works as an efficient triggering mechanism for CMEs. On the other side, the trivial energy release associated with the catastrophe is based on 2D models. In the 3D case, more magnetic energy release is expected since the newly-formed current sheet can be localized in a finite volume. 

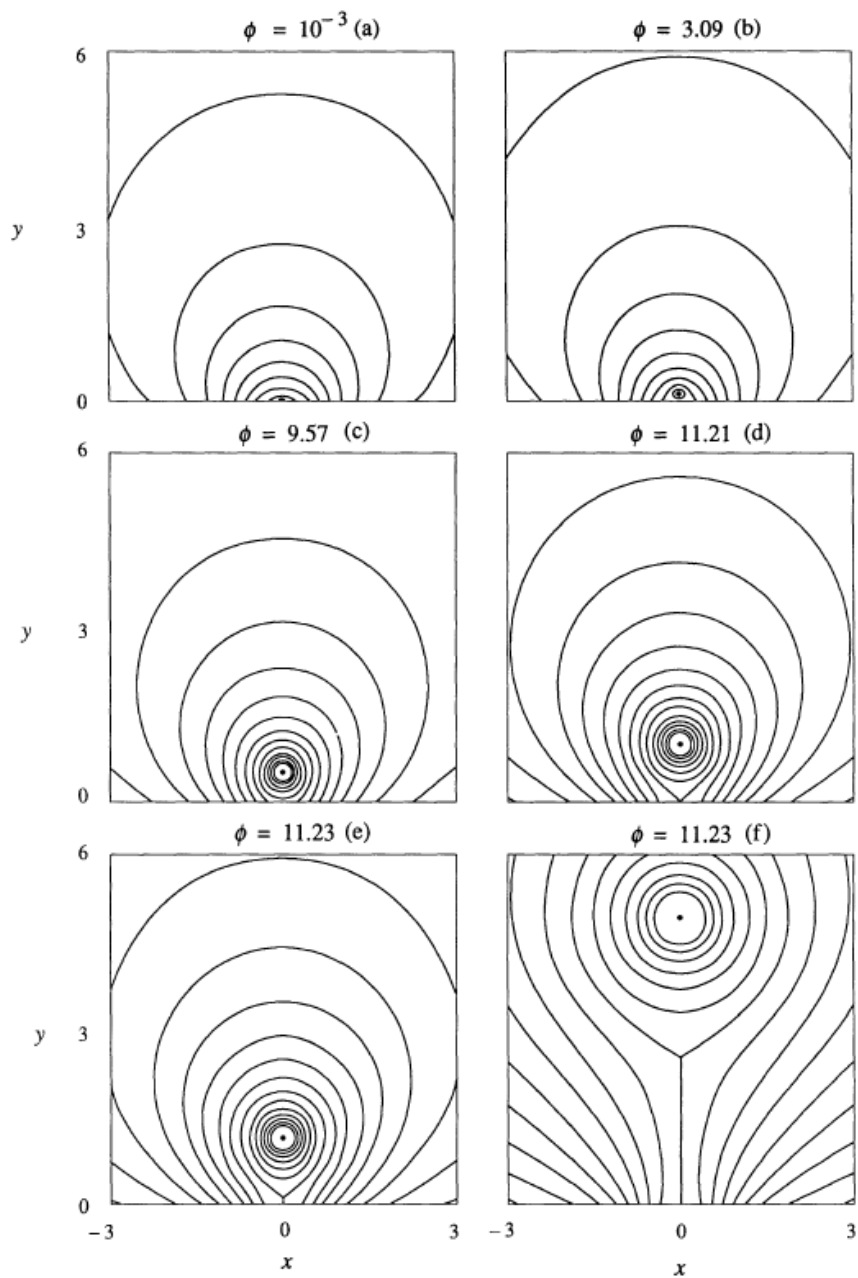

Figure 22: Variation of the equilibrium state of a flux rope system as the amount of the cancelling flux $(\phi)$ increases. From panel (e) to panel (f), a catastrophe takes place (from Forbes and Isenberg, 1991). 
It is mentioned here that such a catastrophe behavior, i.e., the transition from a null-point (at $x=y=0$ ) to a current sheet in Figure 22, reminds us of the X-point instability found by Dungey (1953). Interestingly, Démoulin and Aulanier (2010) found that the critical conditions for the catastrophe also satisfy the instability criterion.

The decay of the background magnetic field is another way to trigger the CME progenitor to deviate from the equilibrium state. The decay of the photospheric magnetic field is often due to the magnetic diffusion that can lead to the formation, as well as the eruption, of flux ropes (Mackay and van Ballegooijen, 2006). The inward diffusion toward the PIL has the effect as the flux cancellation process, and the outward diffusion results in the weakening of the background field. With analytical solutions, Isenberg et al. (1993) found that before $\sigma$ decreases to 0.8136, where $\sigma$ describes the strength of the background magnetic field, the flux rope has only one equilibrium state. However, at $\sigma=0.8136$, there exist two equilibrium states, i.e., a higher energy state and a lower-energy state with a current sheet below the flux rope, which means that the flux rope can transit catastrophically. Lin et al. (1998) extended this work so that the effect of the large-scale curvature of the flux rope is considered. It is found that unlike previous results, flux ropes with large radii are more likely to erupt than ones with small radii. The triggering model due to the decay of the background magnetic field might account for why the peak CME occurrence rate is delayed by 6-12 months with respect to the peak Sunspot number (see Section 2), since the background magnetic field becomes weaker and weaker during the declining phase of the solar cycle.
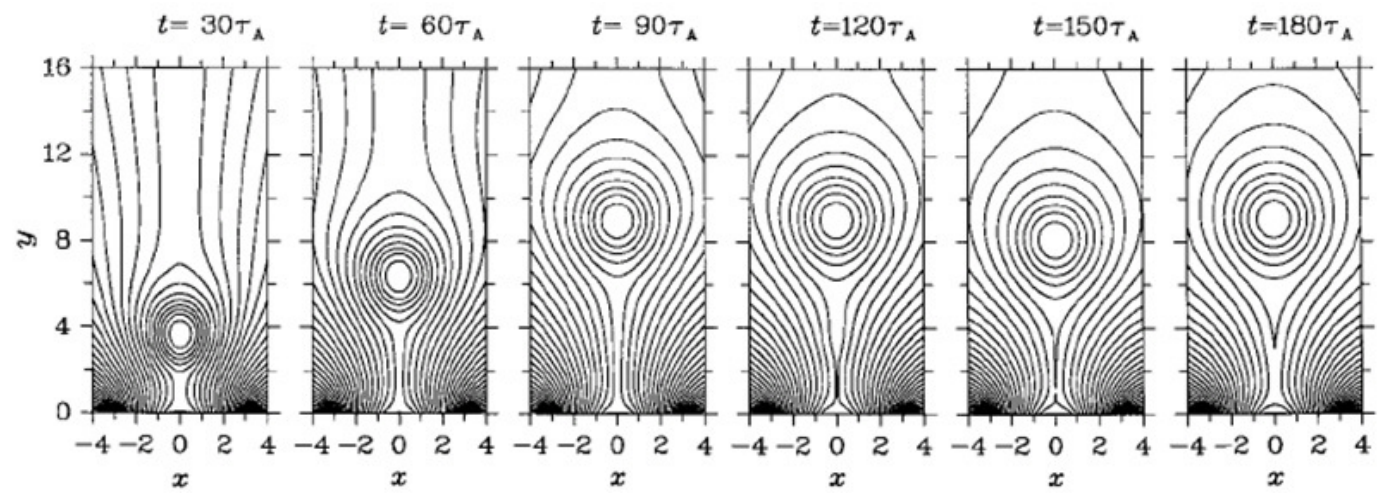

Figure 23: The magnetic evolution showing that the flux rope jumps from an initial equilibrium state $\left(t=30 \tau_{A}\right)$ to a higher state $\left(t=180 \tau_{A}\right)$ when the magnetic shear reaches a critical value (from Hu, 2001).

The increase of the upward magnetic pressure, which can push the CME progenitor to expand, can be realized by either converging motions or the shearing motions. With MHD simulations, Inhester et al. (1992) found that in addition to the shearing motions, the convergent motions can effectively facilitate the formation of a current sheet above the PIL of the magnetic arcade, which would then become resistive unstable. Analytically, Forbes and Priest (1995) found that even without flux cancellation, a flux rope system subject to the photospheric converging motion would also experience a catastrophic behavior. When the half distance $\lambda$ of the dipole decreases from 4 to $>0.97$, the flux rope always possesses only one equilibrium state. However, at $\lambda=0.97$, the flux rope has two equilibrium states, i.e., a higher energystate and a lower energy state with a current sheet below the flux rope. Similar to the background magnetic field decay, such a catastrophe can serve as a nice trigger mechanism for CMEs. It is noticed in Forbes and Priest (1995) that as the background magnetic charges get closer, the flux rope decreases in altitude before the catastrophe appears. However, the 3D MHD numerical simulations by Amari et al. (2003a) indicate that as the converging motion is imposed at the bottom boundary, the flux rope always goes up. The

Living Reviews in Solar Physics

http: //www . livingreviews . org/lrsp-2011-1 
difference is due to the fact that in the analytical study of Forbes and Priest (1995), for any point along the vertical axis, the background field from the two "magnetic charges" increases first (and decreases later). In order to realize a force balance, the line current and its image current should be put closer first (and further away later). One thing has to be kept in mind here: when deriving equilibrium state series subject to changing parameters in the analytical solutions, the frozen-in effect in the ideal MHD might be violated. It is also seen from their comparison that the catastrophe existing in the analytical study is not present in the MHD simulations. It might be due to that there is a "toroidal force" in the 3D flux rope (not existing in the 2D model), which excites the "torus instability" (Török and Kliem, 2007). It also reminds us of the warning that the searching for "loss of equilibrium" solution needs to consider global constraints (Klimchuk and Sturrock, 1989), including the frozen-in effect as mentioned above.

It is interesting to see that, for a system with a flux rope embedded in a bipolar magnetic field, a diversity of changes of the background magnetic field would trigger the catastrophic initiation of the flux rope eruption. As an example, Figure 23 shows the evolution of the loss of equilibrium of the flux rope driven by shearing motions. With the zero plasma $\beta$ and inertialess approximation, Lin et al. (2001) analytically studied the response of the flux rope system to the emerging flux as numerically solved by Chen and Shibata (2000). They found that there are also catastrophic behaviors in the triggering process. However, different from the MHD numerical simulations, the parameter regime in favor of the CME eruptions becomes complicated.

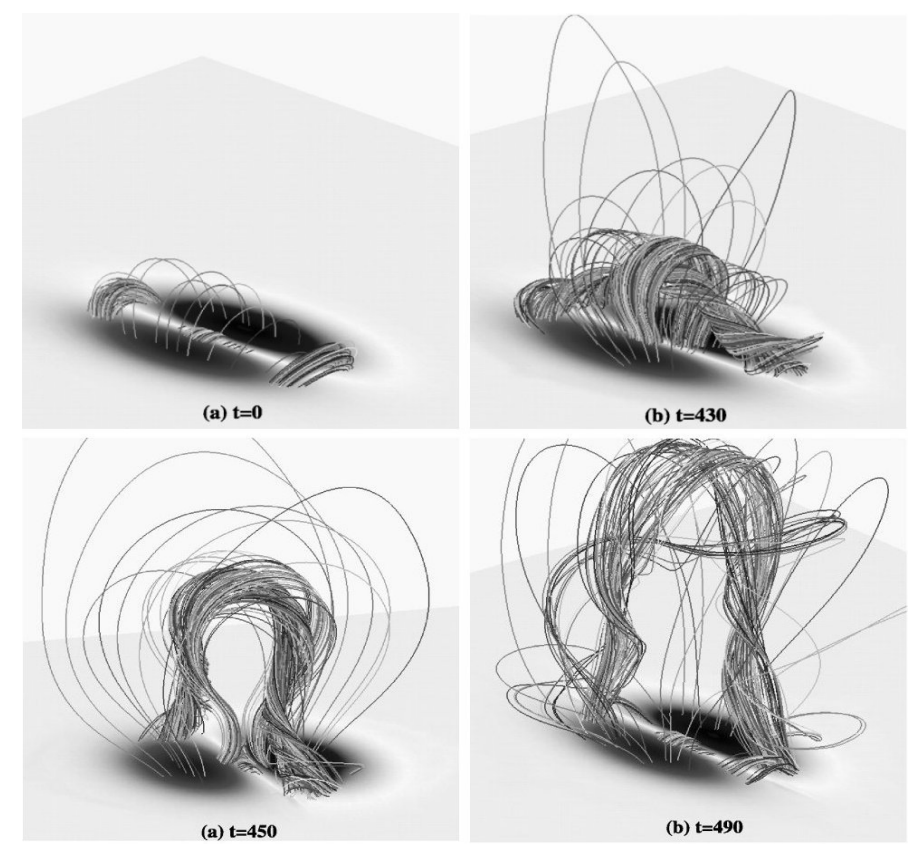

Figure 24: The evolution of the magnetic field in the 3D MHD numerical simulation of Amari et al. (2000), which shows the formation and the ensuing eruption of a twisted flux rope as a simple magnetic arcade experiences shearing motions and the opposite-polarity magnetic emergence (adapted from Amari et al., 2000).

\section{(7) Hybrid mechanisms}

Considering the complexity of the solar atmosphere, such as the unceasing convective motions and magnetic flux emergence, probably several triggering factors may take effect collaboratively. For 
example, with 3D MHD simulations, Amari et al. (2000) studied the initiation of CMEs associated with both shearing motions and flux emergence. As shown by Figure 24, after the shearing motion and the opposite-polarity emerging flux are imposed at the bottom boundary, an initially simple magnetic arcade (upper-left panel) evolves into a twisted flux rope overlaid by an almost potential arcade (upper-right panel). If the magnetic flux of the emerging field is small, the flux rope rises to reach a neighboring equilibrium state. However, if the emerging flux is large enough, the whole system cannot find any equilibrium state, and begins to erupt (bottom panels).

\section{(8) Other mechanisms}

Besides the above-mentioned triggering models, there are some other mechanisms that have not been investigated extensively and quantitatively.

(a) Mass drainage: It is generally assumed that filaments are supported by the Lorentz force against gravity. If a part of filament material drains down to the chromosphere, the filament would lose its equilibrium under the excess Lorentz force (or called magnetic buoyancy, see TandbergHanssen, 1974; Low, 2001). Such a process was studied by Fan and Low (2003) and Wu et al. (2004), and was identified in some eruption events (e.g., Zhou et al., 2006).
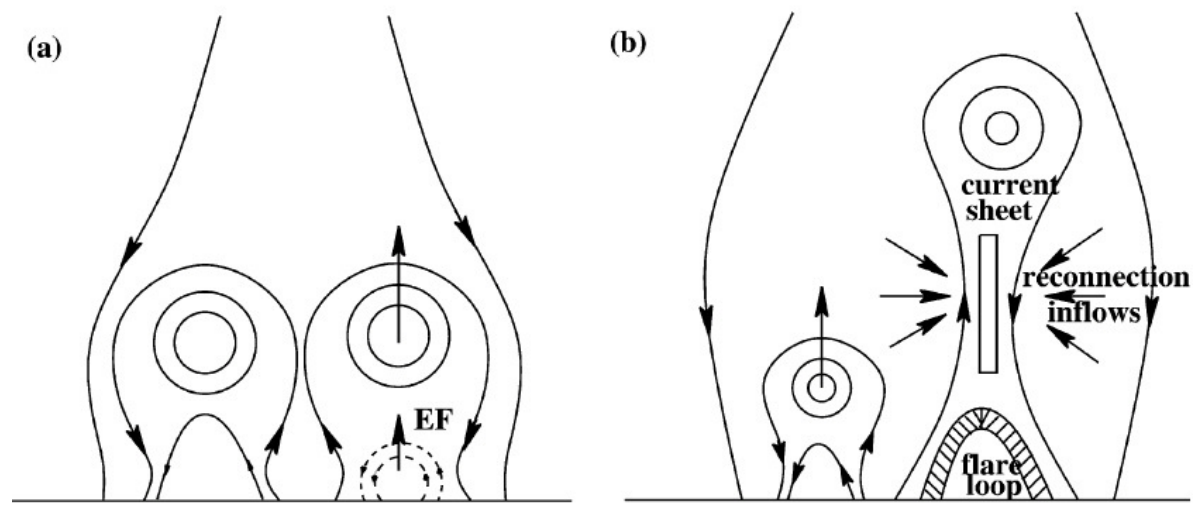

Figure 25: Schematic sketch showing that the reconnection inflow in one CME eruption induces the loss of equilibrium of a neighboring filament (from Cheng et al., 2005).

(b) Sympathetic effect: Moreton waves and/or EIT waves generated by some CME events might trigger the oscillation (Eto et al., 2002; Okamoto et al., 2004) or even the eruption (Ballester, 2006) of a remote filament (such a speculation is worth detailed modelings); The reconnection inflow below an erupting CME may also induce the loss of equilibrium of a neighboring filament, as shown by Figure 25 (see Cheng et al., 2005, for details). In addition, the primary CME pushes aside the background magnetic field, which can also induce the loss of equilibrium of a neighboring filament (Ding et al., 2006).

(c) Solar wind: As illustrated by Figure 26, the CME source region with a closed magnetic configuration is often bounded by open magnetic field, where solar wind is accelerated from being nearly static to several hundred $\mathrm{km} \mathrm{s}^{-1}$. It is possible that the CME source region might be pulled up by the solar wind with the drag force (Forbes et al., 2006) or the pressure gradient (X. Moussas, 2008, private communication). The latter might be called Bernoulli effect, which claims that along a streamline, the higher the velocity the lower the pressure. Note that the dragging effect of the solar wind is significant only when the flux tube extends to a height where the plasma $\beta$ is around unity.

Living Reviews in Solar Physics

http://www . livingreviews . org/lrsp-2011-1 


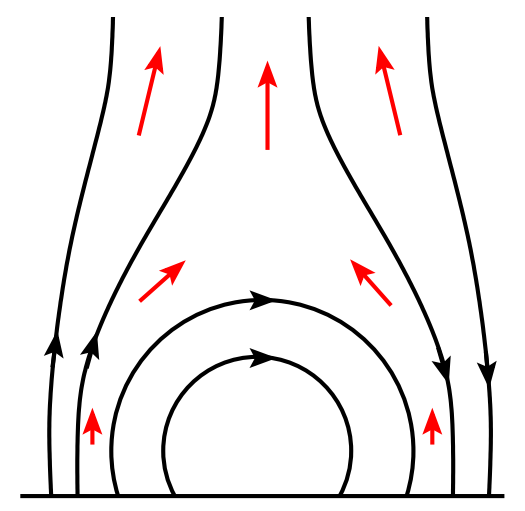

Figure 26: Schematic sketch showing that closed magnetic field is surrounded by an accelerating solar wind background.

\subsubsection{Remarks for the progenitors}

After introducing the triggering mechanisms of CMEs, let us come back to the topic on the CME progenitors. For the slow CMEs that might be accelerated by the solar wind, the energy source is attributed to the solar wind, and the requirement for the CME progenitor is probably that its plasma $\beta$ should be big enough so that the line-tied magnetic field cannot restrain the plasma in the closed field. For the eruptive events, the energy source is attributed to the magnetic energy stored in the progenitor beforehand. The most important question is, then, what physical parameters can well describe the state that the progenitor is ready to be triggered to erupt as a CME.

For the first four triggering processes, such as tether-cutting or flux emergence, the progenitor is not necessarily close to an unstable state, e.g., it might be at a metastable state (Sturrock et al., 2001) (a metastable state means that the system is stable against small perturbations, but it can transit to a lower energy state with a sufficiently large perturbation). During the triggering process, significant changes can be found in observations, such as brightenings or evolving magnetic features. The problem is what parameters can characterize the progenitor that has the potential to erupt.

For the instability and catastrophe-related triggering processes, the progenitor should be close to but still have not reached the criteria of the instability. Therefore, future research might be focused on the quantitative determination of the criteria of the related instabilities based on the real observational data, especially the vector magnetograms and the extrapolated coronal magnetic field.

Based on multiwavelength observations, especially the photospheric vector magnetograms, researchers tried to find out some empirical measures that can well represent the nonpotentiality of CME-productive regions. For instance, Falconer et al. (2002) found that three measures are well correlated with the CME productivity: $L_{S S}$, the length of strong-shear, strong-field main neutral line; $I_{N}$, the net electric current arching from one polarity to the other; and $\alpha=\mu I_{N} / \phi$, a flux-normalized measure of the field twist.

From the theoretical point view, magnetic free energy is a parameter directly related to the nonpotentiality of the pre-CME metastable state. However, it is not rare to see that the magnetic free energy is increasing after one CME due to the Poynting flux through the solar surface, without any ensuing eruptions for hours (e.g., Jing et al., 2009). In this sense, the combinative study on the internal cause, e.g., the free energy, and the external cause, e.g., a suitable trigger, becomes crucial. Magnetic helicity, which is an almost conservative quantity even in a resistive process (Berger, 1984), could be a better parameter. The coronal electric current helicity, as well as 
the magnetic helicity, is cyclic invariant (Seehafer, 1990), therefore, CMEs are the main process to remove the otherwise accumulating helicity in active regions (Rust, 1994; Low, 1996). (The interchange reconnection between the closed and the open fields is another way.) Zhang et al. (2006) conjectured that for a given boundary condition, there may exist an upper bound on the magnetic helicity, which changes with the boundary conditions (Zhang and Flyer, 2008). According to the conjecture, any further pumping of magnetic helicity (or evolving boundary conditions) into the magnetic system at the threshold would lead to the eruption of a CME. As a support from observations, Nindos and Andrews (2004) found that in a statistical sense the preflare value of $\alpha$ (the force-free factor) and coronal helicity of the active regions producing big flares that do not have associated CMEs are smaller than those producing CME-associated big flares. On the other hand, Phillips et al. (2005) argued that CME eruptions occur at a fixed amount of free energy in the corona, no matter the magnetic helicity is large or zero. Also a little surprisingly, the CME eruption may be triggered by a process along with the injection of magnetic helicity that has an opposite sign to the source active region, as demonstrated by Kusano et al. (2002) and Wang et al. (2004). Therefore, the role of magnetic helicity in triggering CMEs remains a controversial problem (see Démoulin, 2007, for a review).

Anyway, we are far from being able to predict where and when a CME is going to erupt.

\subsection{Acceleration and propagation}

From above it is seen that, as the closed magnetic structure is initiated to rise, a current sheet is newly formed above the magnetic neutral line. The current sheet exerts a resisting force over the CME progenitor. If magnetic reconnection is then excited in the current sheet, the reconnection leads to the rapid energy release from magnetic energy to thermal, kinetic, and nonthermal energies, which is just the picture of the CSHKP model: the downward reconnection outflow collides with the flaring loops which are line-tied to the solar surface, whereas the upward reconnection outflow pushes the core field of the CME source region, which is then accelerated to move up. The upmoving core field, e.g., a flux rope, further stretches up the overlying magnetic field lines, leading to the large-scale magnetic restructuring along with the mass ejection. Such a resistive MHD paradigm is probably applicable to a majority of CME events, especially those energetic ones, as evidenced by the strong relationship between CMEs and flares. If magnetic reconnection is not excited in the newly formed current sheet, from a theoretical point of view, the CME progenitor, after being triggered, may also erupt via an ideal MHD process, e.g., the ideal MHD instabilities in the low plasma beta case or the dragging force from the solar wind, if the outward force can overtake the resisting force from the current sheet. Such an ideal MHD paradigm is particularly discussed in Section 4.1.

The CME propagation was mainly observed by imaging telescopes. However, in imaging observations, it is always a problem to distinguish mass motions from waves and apparent motions (for instance, the ribbon separation in solar flares is a kind of apparent motion). Regarding the CMEs, we describe the acceleration and propagation of their components separately, i.e., the bright core, the cavity, and the frontal loop. The rising of the bright core, i.e., the filament, is definitely a mass motion. The CME frontal loop, however, might be much more complicated (see discussions in Section 4.4).

\subsubsection{Bright core}

Kahler et al. (1988) traced four CME/filament eruption events, and found that the $\mathrm{H} \alpha$ filaments in all cases were moving upward slowly before the flare onset, indicating that the CMEs have already been triggered before the flare. Moreover, they found that two of the filaments showed strong acceleration during the impulsive phase of the flares, indicating that the main magnetic

Living Reviews in Solar Physics

http://www. livingreviews . org/lrsp-2011-1 
reconnection plays an important role in accelerating the filaments (by "main reconnection" here we distinguish it from the localized reconnection in the triggering process).

EIT $304 \AA$ images can trace the erupting filament further out, and it was found that the filament rises with an almost constant velocity after the impulsive acceleration phase (e.g., Joshi and Srivastava, 2007).

The upward reconnection outflow would hit the bottom of the flux rope, forming SXR plasmoids just below the filament (Ohyama and Shibata, 2008). Therefore, it is not surprising that the SXR plasmoid, which traces the filament, would also show a strong acceleration during the flare impulsive phase, as revealed by Ohyama and Shibata (1997). It is also expected that the SXR plasmoid also becomes a part of the CME bright core. In this case, the plasmoid motion, moving along with the filament, is different from the upward reconnection outflow, with the latter being much faster.

The erupting filament, so as the bright CME core, may have inverse or normal polarities. While little attention was paid to the dynamics of the eruption of normal-polarity filaments, a lot of efforts have been made to investigate the dynamics of the filaments with the flux rope configuration. Note that, in the case of the flux rope configuration, it is believed that the filament is located near the bottom of the flux rope (Kuperus and Raadu, 1974). The modeling of the flux rope dynamics includes analytical formulations and MHD simulations.

Similar to Anzer (1978), the dynamics of the flux rope in the flux injection model of Chen (1996) is determined by Newton's second law for simplicity, i.e., $M \frac{d^{2} Z}{d t^{2}}=F_{R}$, where $M$ is the total mass per unit length of the flux rope, $Z$ is the apex height of the flux rope axis, $F_{R}$ is the integrated force including the drag term related to the solar wind, and $t$ is time. The solution reproduces the impulsive acceleration of CME bright core, which is followed by an almost uniform propagation as revealed by the right panel of Figure 19.

With the assumption that the plasmoid (or flux rope) is accelerated solely by the momentum of the reconnection outflow, Shibata and Tanuma (2001) derived an analytical solution to the motion of the plasmoid, i.e.,

$$
V_{p}=\frac{V_{A} \exp (\omega t)}{\exp (\omega t)-1+V_{A} / V_{0}},
$$

where $V_{p}$ is the plasmoid velocity, $V_{A}$ is the Alfvén speed of the ambient corona, $V_{0}$ is the initial velocity of the plasmoid before reconnection, $\omega=\rho V_{A} /\left(\rho_{p} L_{p}\right), \rho$ is the density of the ambient corona, $\rho_{p}$ the density of the plasmoid, $L_{p}$ the vertical length of the plasmoid, and $t$ the time. As seen in Figure 27, the simple model, for the case of $V_{0}=0.01 V_{A}$, can also reproduce the typical observational features, i.e., the CME core, or erupting prominence, is strongly accelerated in the impulsive phase of the flare, and then moves up with an almost constant velocity. Such a model is rather simple, where the restraining force from the coronal loops overlying the flux rope is neglected.

A more sophisticated approach is the circuit model by Martens and Kuin (1989), which is equivalent to the modeling of inertialess MHD equations. Their model can be regarded as the quantitative version of the CSHKP model. It turned out to well explain the impulsive acceleration of the flux rope, while fail to explain the later propagation phase. This model was improved by Lin and Forbes (2000), as shown in Figure 28. Their analytical solution reproduced the three-phase feature for the CME core very well, i.e., a slow rise in the preflare phase, a strong acceleration in the flare impulsive phase, and the final eruption with a nearly constant velocity in the gradual phase.

Numerical simulations of the full set of the MHD equations provide the CME dynamics that can be better compared with observations. The 2D simulations by Chen and Shibata (2000) showed that, as the flux rope loses its equilibrium due to the emerging flux (see Section 3.4.2), a current sheet forms near the null point below the flux rope. As anomalous resistivity sets in, magnetic reconnection proceeds in the current sheet. On one hand, it forms a cusp-shaped flare below the reconnection site as described by the standard flare model. On the other hand, with 


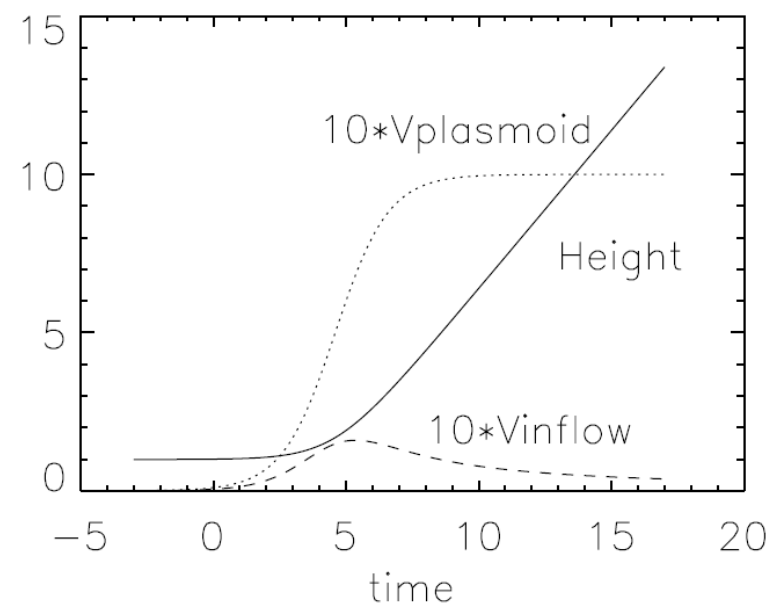

Figure 27: Temporal variations of the plasmoid velocity ( $V_{\text {plasmoid }}$, dotted line), its height (solid line), and inflow velocity ( $V_{\text {inflow }}$, dashed line) in the analytical model of Shibata and Tanuma (2001).

reconnection, the constraint of the closed field lines overlying the flux rope is removed, and the flux rope is accelerated, as depicted by Figures 17 and 18. The time evolution of the flux rope height is compared with that of the reconnection rate in Figure 29. It is seen that the flux rope is intensively accelerated near the peak of the reconnection rate, i.e., the flare impulsive phase, after which the flux rope rises with an almost invariable velocity, a typical feature found in the erupting filament observations (Kahler et al., 1988; Sterling and Moore, 2005).

The consistency of the above models with observations of many CMEs implies that magnetic reconnection is the key to linking the violent $\mathrm{CME}$ and flare eruptions as a unity. However, as we will see in Section 4.1, some factors other than reconnection may also contribute to the acceleration of CMEs, e.g., ideal MHD instabilities (Amari et al., 2004; Fan and Gibson, 2007), which is the reason why some events present continual acceleration before and after the impulsive phase (e.g., Kahler et al., 1988). They are these factors that might accelerate some CMEs without the involvement of magnetic reconnection, e.g., via ideal MHD instabilities such as those discussed in Section 3.4.2. In MHD simulations, numerical resistivity is generally unavoidable, therefore, it is still premature to see the ideal MHD models of CME eruptions. Instead, the corresponding dynamics of CMEs is often studied by solving the motion equation of the flux rope under the integrated force, especially the toroidal force. As demonstrated by Chen (1989, see Figure 19) and Kliem and Török (2006), the flux rope can indeed be accelerated and then approach an almost constant velocity. It is pointed out here that one thing is missing in these works, i.e., the existence of a newly-formed current sheet below the flux rope, which resists the flux rope from further erupting.

It is noted in passing that the circular flux rope in the low corona may evolve into a pancake structure in the interplanetary space (Riley et al., 2003; Manchester IV et al., 2004).

\subsubsection{Cavity}

An expanding cavity often immediately follows the CME frontal loop, with the bright core being embedded in (Illing and Hundhausen, 1985). The white-light deficit means that the density in the cavity is relatively low. The density depleted cavity would also be weak in EUV wavelength, as illustrated by Chen et al. (2000) and Chen (2009a). The mass outflow in the dimming region was measured by spectroscopic observations (Harrison and Lyons, 2000; Harra and Sterling, 2001),

Living Reviews in Solar Physics

http://www. livingreviews.org/lrsp-2011-1 


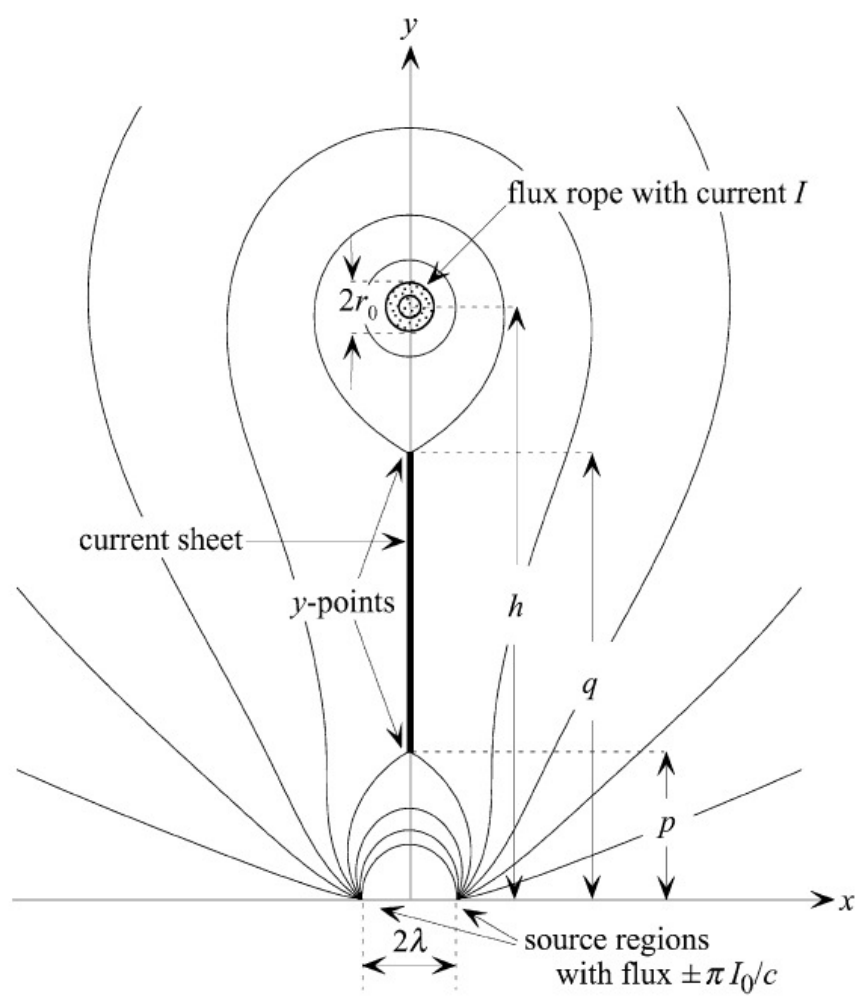

Figure 28: Diagram of the magnetic configuration including an erupting flux rope and a current sheet between the flux rope and the reconnected flare loop used for the setup of the analytical solution by Lin and Forbes (2000).

which was interpreted as the result of magnetic field line stretching pushed by the erupting flux rope (Chen et al., 2002, 2005c).

The CME cavity, which is observed above the solar limb, should have disk counterpart. Theoretically, Chen and Fang (2005) proposed that whereas the CME frontal loop corresponds to the EIT wave front on the solar disk, the CME cavity corresponds to the extended dimmings, which follow the EIT wave fronts. With the gap of the fields of view between the SOHO/LASCO coronagraph and SOHO/EIT, Thompson et al. (2000) speculated that the angular span of EUV extended dimmings is roughly the same as the corresponding CME. With the overlapping fields of view of Mauna Loa Solar Observatory (MLSO)/MK4 coronagraph and SOHO/EIT, Chen (2009a) verified that EIT wave front, which borders the extended dimmings, is the EUV counterpart of the CME frontal loop, while EUV extended dimmings are the disk counterpart of the CME cavity. Such a result is understandable since both EIT wave fronts and CME frontal loops are characterized by density enhancement, whereas both EUV extended dimmings and the CME cavity are characterized by density depletion.

It is noted that, even before CME eruption, a coronal cavity may be visible surrounding the torpid prominence (e.g., Vaiana et al., 1973; Hudson et al., 1999), as a part of the helmet streamer (Illing and Hundhausen, 1986). Consensus has not been achieved regarding the relationship between the pre-eruption streamer cavity and the CME cavity. While it was claimed that the streamer cavity simply swells and erupts to become the CME cavity (Illing and Hundhausen, 1986; Gibson et al., 2006b), an alternative explanation is that the CME cavity results from successive stretching of the closed magnetic field lines from the inner field to the outside (Delannée and Aulanier, 1999; 


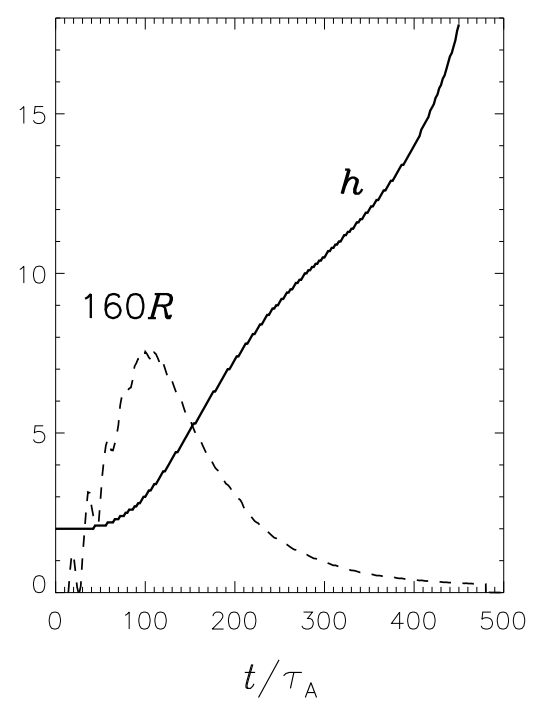

Figure 29: Temporal evolutions of the flux rope height $(h)$ and the magnetic reconnection rate $(R)$ in the numerical simulation of Chen and Shibata (2000), which shows that the strong acceleration of the flux rope is coincident with the peak reconnection rate.

Chen et al., 2002, 2005c). I tend to believe that the streamer cavity is a steady structure, which is believed to correspond to the filament channel (Engvold, 1989) and is, therefore, related to the filament formation. However, the CME cavity is a dynamic structure, with its footprints expanding laterally as more and more field lines are stretched up, i.e., the streamer cavity is only the initial part of the CME cavity, and more and more overlying field lines are involved into the cavity during the CME eruption.

Two issues were often ignored about the CME cavity. The kinetic energy of the cavity is often neglected (Vourlidas et al., 2000), probably because the plasma density of the CME cavity (or the extended dimmings) is small. However, the cavity volume is very large, where outflows with a high velocity are often induced (Harra and Sterling, 2001), therefore, to which extent it may contribute to the total kinetic energy should be clarified. On the other side, as the gas pressure in the cavity decreases during the CME eruption, the pressure gradient is expected to drive outflow from the transition region and even chromosphere (Jiang et al., 2003; Jin et al., 2009). This process, in addition to the interchange reconnection between the stretched magnetic field and small coronal loops (Attrill et al., 2008), refills the depleted corona, making the coronal dimming recover to normal in tens of hours.

\subsubsection{Frontal loop}

The kinematics of the frontal loop, sometime called leading edge, was extensively studied. Combining the Mark III (MK3) K coronameter at Mauna Loa Solar Observatory and the SMM space-borne coronagraph, St Cyr et al. (1999) found that among the 141 CME events, the frontal loop in 87\% of the sample showed a positive acceleration, which is averaged at $0.264 \mathrm{~km} \mathrm{~s}^{-1}$. The strong acceleration happens generally below $2 R_{\odot}$, beyond which CMEs move with an almost constant velocity. With the unprecedented high-resolution and wide-view observations by SOHO/LASCO coronagraph, Zhang et al. (2001a) investigated four CME events covered down to $1.1 R_{\odot}$ and found that the CME frontal loop propagation can be described in a three-phase scenario, as illustrated

Living Reviews in Solar Physics

http: //www . livingreviews . org/lrsp-2011-1 
by Figure 30:

(1) The initiation phase, when the frontal loop rises slowly with a velocity of $\sim 80 \mathrm{~km} \mathrm{~s}^{-1}$ for tens of minutes before the main flare. During this phase, the CME is being triggered.

(2) The impulsive acceleration phase, when the frontal loop is accelerated rapidly. This phase lasts several to tens of minutes, which is coincident with the impulsive phase of the main flare.

(3) The propagation phase, when the frontal loop moves with an almost constant velocity.

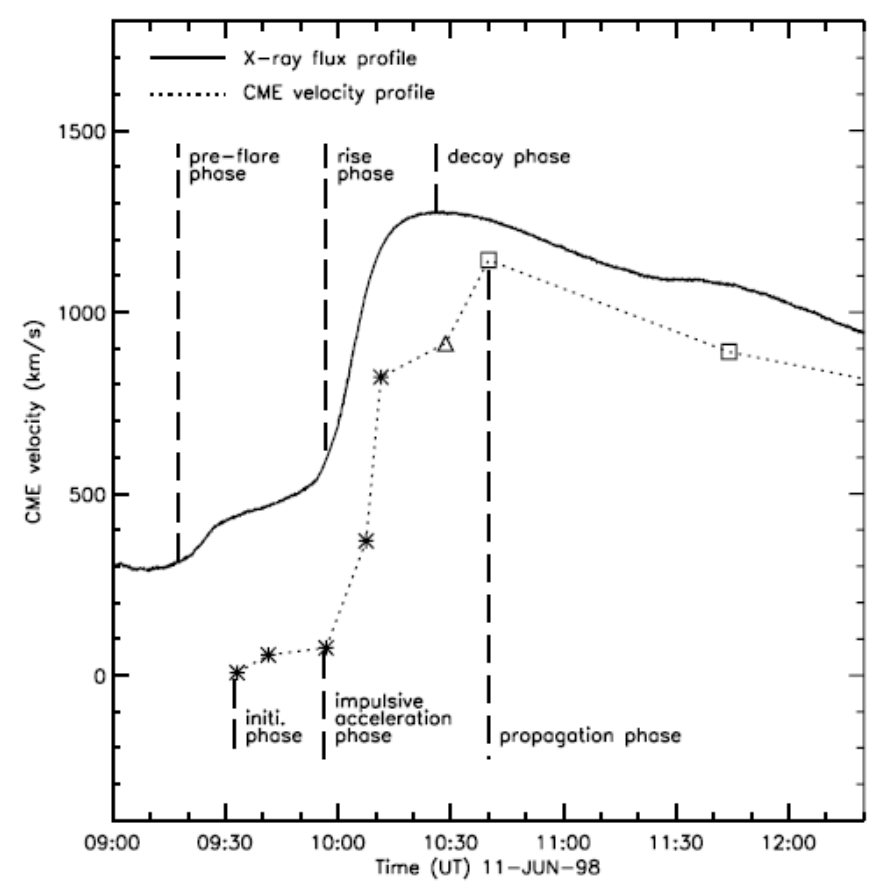

Figure 30: Temporal evolutions of the SXR flux of the flare (solid line) and the propagation velocity of the CME frontal loop (dotted line), showing a three-phase pattern (from Zhang et al., 2001a).

Of course, the CME frontal loop also shows smaller acceleration or deceleration in the propagation phase. Statistical study by Zhang and Dere (2006) indicates that the main acceleration in the impulsive phase widely ranges from $2.8 \times 10^{-3}$ to $4.464 \mathrm{~km} \mathrm{~s}^{-2}$, with an averaged value at $0.331 \mathrm{~km} \mathrm{~s}^{-2}$; the corresponding main acceleration duration widely ranges from 6 to $1200 \mathrm{~min}$, with an averaged value at $180 \mathrm{~min}$. On the other hand, the residual acceleration in the propagation phase ranges from -131 to $52 \mathrm{~m} \mathrm{~s}^{-2}$, with an averaged value at $0.9 \mathrm{~m} \mathrm{~s}^{-2}$, much smaller than the main acceleration. They also found a scaling law between the main acceleration $(a)$ and its duration $(T)$, i.e., $a=10^{4} T^{-1}\left(\mathrm{~m} \mathrm{~s}^{-2}\right)$, where $T$ is in unit of min.

As the CMEs propagate in the interplanetary space, it is found that fast CMEs decelerate and slow CMEs accelerate, so that the ICME velocity tends to approach the ambient solar wind speed. Gopalswamy et al. (2000) derived an empirical formula for the CME propagation from the coronagraph field of view to $1 \mathrm{AU}$ :

$$
a\left[\mathrm{~m} \mathrm{~s}^{-2}\right]=1.41-0.0035 u\left[\mathrm{~km} \mathrm{~s}^{-1}\right],
$$

where $a$ is the mean acceleration in the interplanetary space and $u$ is the CME propagation velocity measured in the coronagraph field of view. The asymptotic solution of the above formula, as $a$ approaches 0 and $u$ becomes constant, is $u=1.41 / 0.0035=403 \mathrm{~km} \mathrm{~s}^{-1}$. 
In contrast to the bright core and the cavity, the CME frontal loop is actually much less understood. It is generally taken for granted that the CME frontal loop propagation is regarded as a mass motion, and in most modelings, the CME frontal loop was generally considered as the apex of the flux rope (see Forbes et al., 2006, for details). However, the propagation of the CME frontal loop might not be a mass motion (see Section 4.4 for a completely different explanation). For instance, as described in Forbes (2000), the CME frontal loop may be a plasma pileup region rather than the flux rope. Therefore, we do not explicate the modeling of the CME frontal loop here, leaving the topic to be discussed in Section 4.4.

\subsubsection{Lateral expansion}

The CME propagation features mentioned in the previous two subsections are focused on the radial direction. In this subsection, we briefly talk about the lateral expansion of CMEs.

Lacking the observations in the low corona, CME evolutions above $2 R_{\odot}$ gave an impression that CMEs generally have a fixed angular width. For instance, Figure 25 of Schwenn (2006) shows that the $60^{\circ}$ cone angle and the general shape of the 2000 February $27 \mathrm{CME}$ event are maintained during the whole 12 hour passage through the LASCO field of view. This maintained "self-similarity" is characteristic for most CMEs (Low, 2001), and is also the foundation for the cone model that describes the CME geometry (Howard et al., 1982; Fisher and Munro, 1984). However, low corona observations indicated that CMEs experience significant lateral expansion in the early stage. For example, St Cyr et al. (1999) found that the average width of CMEs in the lower field of view of the MK3 coronagraph is $12^{\circ}$ smaller than that measured in SMM. It is just due to such an expansion, which is often asymmetric on the two sides, that a CME becomes much wider than the accompanying flare, and the flare is often near one footpoint of the CME, rather than being centered under the CME (Harrison, 1986). Such an expansion of the CME and its asymmetry to the solar flare can be understood as follows: as the CME frontal loop was suggested to be formed due to successive stretching of magnetic field lines (Chen, 2009a), it is expected that, if the background magnetic field is asymmetric, a larger area of magnetic field is involved to form the CME on the side with weaker magnetic field, and we are ready to see the expanding CME covers a larger span on this weak field side as depicted by Figure 31. Therefore, an asymmetric ambient magnetic configuration naturally results in a CME asymmetric to the underlying flare.
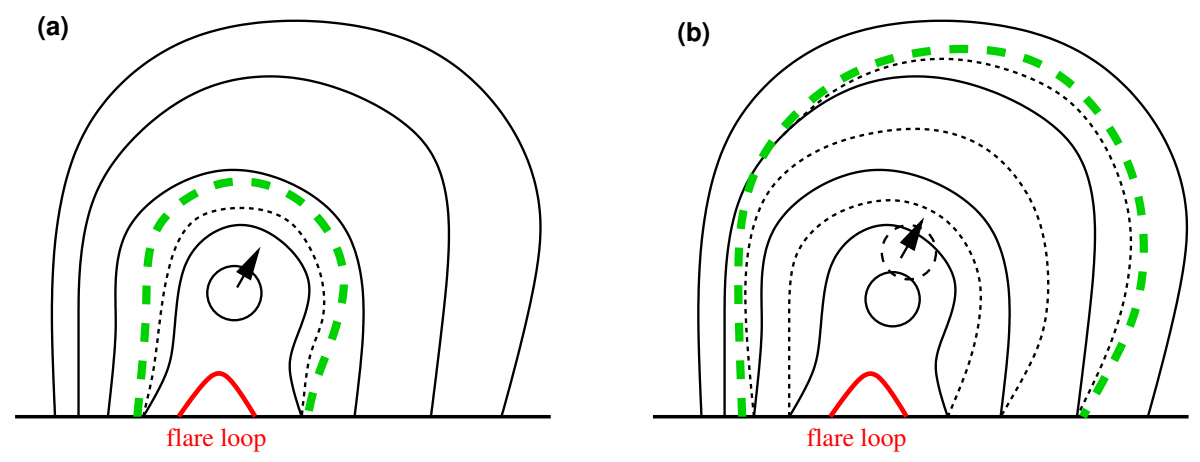

Figure 31: Schematic sketch showing how a CME frontal loop expands to be asymmetric to the underlying flare in the early stage. The black solid lines correspond to the initial magnetic field, the black dashed lines to the deformed magnetic field, and the green dashed lines to the CME frontal loop formed by the successive stretching of magnetic field lines. The arrows indicate that the flux rope is erupting.

It is interesting to note that in most CME/flare modelings, only a central bipolar magnetic structure erupts as a CME. Even in the magnetic breakout model where a quadrupolar configu-

Living Reviews in Solar Physics

http://www. livingreviews . org/lrsp-2011-1 
ration is present (Antiochos et al., 1999), the ejected magnetic structure is still the bipolar field near the central neutral line. However, there often exist interconnecting loops above the source region of the CME eruption, and the erupting bipolar structure would interact with the overlying interconnecting loops. If the overlying loops have roughly the same magnetic orientation as the erupting bipolar structure (maybe with a small angle), the latter would pull up the overlying loops, leading to a global CME (Zhukov and Veselovsky, 2007) as illustrated in Figure 32 (Delannée and Aulanier, 1999; Delannée et al., 2007) and numerically modeled by Roussev et al. (2007). If the overlying or even lateral loops have roughly opposite magnetic orientation as the erupting bipolar structure, interchange reconnection could happen between them, leading to a CME with a re-organized morphology (Attrill et al., 2006; Gibson and Fan, 2008).

All these three situations, i.e., the asymmetric expansion of the source active region, its merging with the overlying interconnecting loops, and the reconnection with the ambient or overlying field, could happen in combination (Cohen et al., 2009). They may explain why many flares are located near one footpoint of the corresponding CMEs.

(a)

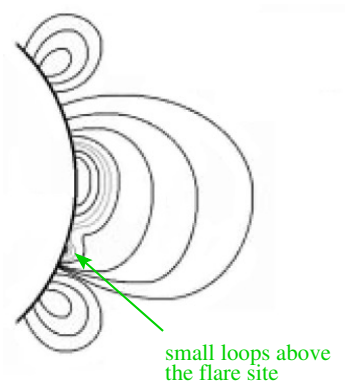

(b)

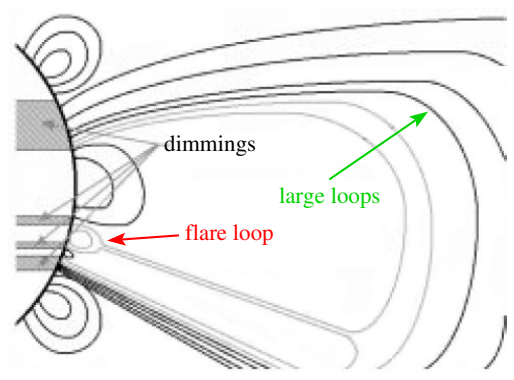

Figure 32: Schematic sketch showing how a CME expands to a global scale as it pushes up the overlying interconnecting loops. The gray lines corresponds to small-scale magnetic field, black lines to the interconnecting lines (adapted from Delannée and Aulanier, 1999).

\subsubsection{CME-associated shocks}

Similar to the launch of a rocket, the eruption of a CME would also drive a piston-driven shock wave ahead of it. The shock, which can locally accelerate electrons to $\sim 10 \mathrm{keV}$ and hence excite in situ plasma emissions, was observed as type II radio bursts (Wild and McCready, 1950). Such a piston-driven shock was identified in the interplanetary type II radio bursts at $\sim 290 \mathrm{kHz}$ (Malitson et al., 1973), as well as the decametric coronal type II radio bursts at $\sim 2.5 \mathrm{MHz}$ (Reiner et al., 2000). It is still on debate whether such a piston-driven shock may show up at metric wavelengths (Vršnak and Cliver, 2008).

The white-light imaging signature of this piston-driven shock should be the so-called "CME forerunner" found by Jackson and Hildner (1978), which was later inferred based on the streamer deflections (Sheeley Jr et al., 2000) and clearly detected by Vourlidas et al. (2003) and Eselevich and Eselevich (2008), as shown by Figure 33. The spectroscopic properties of the CME-driven shock are well documented by Raymond et al. (2000), Mancuso et al. (2002), and Ciaravella et al. (2006). Such a piston-driven shock extends down to the solar surface, especially in the early stage. Generally speaking, the strength of such a bow shock would be the strongest at the top since the driver, the erupting flux rope, is moving upward. Gopalswamy et al. (2001) proposed that, however, in a non-uniform corona, the shock strength at one flank may also be strong enough to form a second type II radio burst, e.g., at metric wavelengths. 


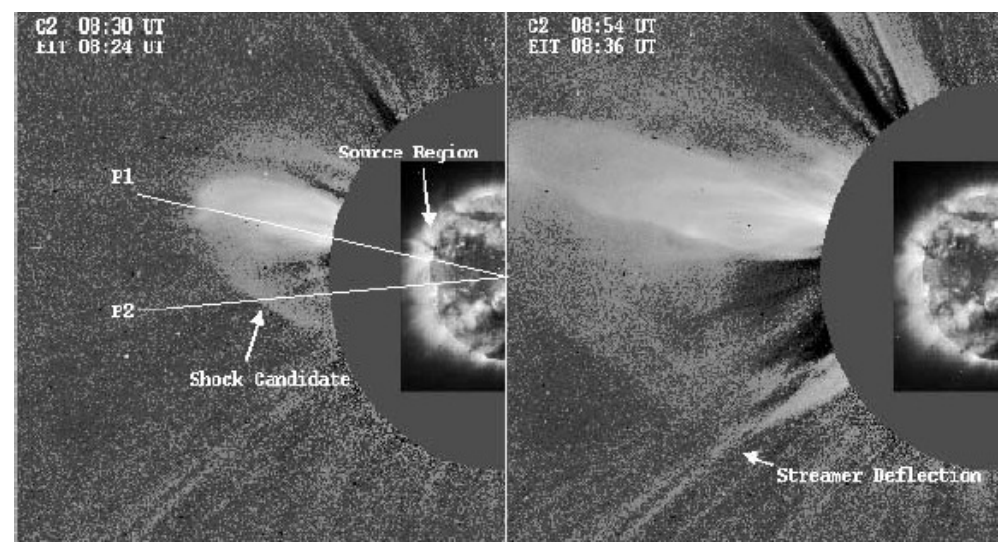

Figure 33: The LASCO/C2 white-light images showing a faint bow shock straddling over the 1999 April 2 CME. The solar disk is revealed by the EIT $195 \AA$ images (adapted from Vourlidas et al., 2003).

The footpoint of the shock sweeping the chromosphere was proposed to generate $\mathrm{H} \alpha$ Moreton waves (Chen et al., 2002, 2005c), which were originally explained due to the flare-induced blast waves (Uchida, 1968). It is expected that the shock strength at the footpoint decays drastically as the piston, i.e., the erupting flux rope, moves higher and higher. This may explain why Moreton waves are visible for a relatively short distance.

According to the standard CME/flare model, it is expected to see another two fast-mode termination shocks associated with the CME/flare eruption, i.e., the upward reconnection outflow collides with the flux rope, forming a reverse fast-mode shock, and the downward reconnection outflow collides with the flaring loops, forming another fast-mode shock, as illustrated in Figure 34. The termination shock below the flux rope apparently propagates up along with the erupting CME core. This shock may also excite type II radio bursts, and Magara et al. (2000) applied it to interpret the metric type II burst below the faster-moving decametric burst, which was found by Reiner et al. (2000). Similarly, the termination shock at the flare loop top might be observed as high-frequency slowly drifting radio structures found by Karlický et al. (2004), though they interpreted it as accelerated electrons trapped in the erupting plasmoids.

Another open question is whether the pressure pulse of the flaring loop can generate a blast wave which produces metric type II bursts. According to the standard CME/flare model shown in Figure 34, heat and nonthermal particles are transferred down from the reconnection site to the solar surface, the resulting high pressure drives chromospheric evaporation, with hot and dense plasma filling the flare loop. It was suggested that the high pressure would excite a blast shock wave, which accounts for the metric type II radio burst and Moreton waves (Uchida, 1974; Wagner and MacQueen, 1983; Vršnak and Lulić, 2000). Some authors have a different view (e.g., Cliver et al. 1999; Chen et al. 2002, 2005c; see Vršnak and Cliver 2008 for a review), and numerical simulations of magnetic reconnection (e.g., Chen et al., 1999) did not show a blast wave from the flaring loop, even when chromospheric evaporation is reproduced (Yokoyama and Shibata, 2001). This question definitely deserves further clarification.

Finally, we would like to comment on the formation of the piston-driven shock straddling over the CME. It was often mentioned that the shock forms when the velocity of the ejecta becomes larger than the local Alfvén wave or fast-mode wave speed. We want to emphasize that this is not necessary since a compressible simple wave may steepen and form a shock wave nonlinearly (Vršnak and Cliver, 2008), especially for a simple wave with a short wavelength, e.g., in the case when a CME is suddenly accelerated. 


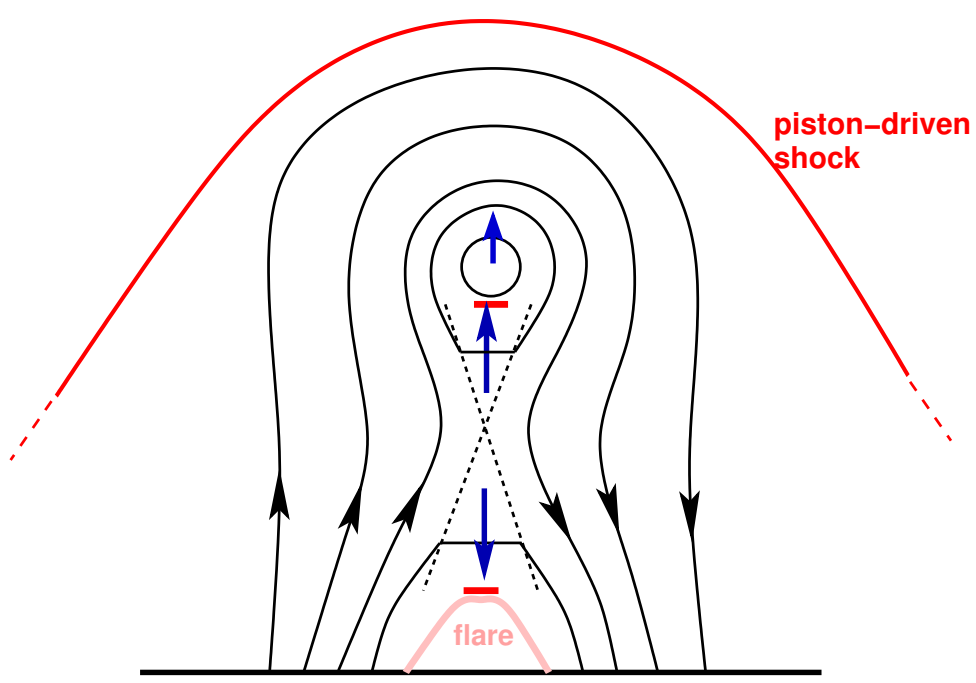

Figure 34: Schematic sketch showing the possible shocks associated with CMEs in the standard model. Besides the piston-driven shocks, another two fast-mode termination shocks, which are formed as reconnection outflows (long blue arrows) collide with the flux rope and the flare loop, are indicated by red bars. Black solid lines correspond to the magnetic field, dashed lines to the slow-mode shock fronts. 


\section{Debates}

In this section, we discuss some key topics that are still under hot debate.

\subsection{Is magnetic reconnection necessary?}

CMEs are believed to be due to that closed magnetic field lines are stretched up to the interplanetary space, as confirmed by the counterstreaming electron flux (Gosling et al., 1987). It is misleading that the field lines are widely called to be open in the early phase of the CME eruption in the literature. Actually, the field lines are being stretched up all the way from the corona to the interplanetary space, unless they occasionally reconnect with ambient open magnetic field (Attrill et al., 2006). Along with the usage of the word "open", it was often assumed that before the magnetic reconnection, which accounts for the solar flare, the closed field lines were already opened (Barnes and Sturrock, 1972; Pneuman, 1981; Low, 1994), i.e., owing to loss of equilibrium or some kind of instability, the closed magnetic field lines erupt to infinity, forming an open magnetic configuration with a current sheet standing above the magnetic neutral line. As the second step, the ensuing magnetic reconnection of the current sheet leads to the solar flare. Regarding this process, Aly (1991) and Sturrock (1991) conjectured that, if all the magnetic field lines are simply linked to the solar surface, the total energy of any force-free magnetic field cannot exceed that of the open field having the same flux distribution on the solar surface. This was later called "Aly-Sturrock" constraint, which means that the first step in the above models may not be feasible in the ideal MHD framework.

The observational fact that solar flares generally occur tens of minutes after the CME is initiated implies that magnetic reconnection often takes place soon after the CME progenitor is stretched up in the low corona (well before it is stretched up to be open field), and the "Aly-Sturrock" constraint is not applicable to these resistive MHD processes. Actually most resistive MHD numerical simulations have been performed to reproduce CMEs like this, without the necessity to consider the constraint.

Even in the ideal MHD framework, there are still several ways to circumvent the Aly-Sturrock constraint, allowing the initial magnetic field to have the energy higher than that of the open field, which means that the transition from the initial closed field to the open field is feasible from the energy point of view, and an eruption driven by ideal MHD processes is feasible:

(1) The initial magnetic field is not simply linked to the solar surface, e.g., it contains detached magnetic field lines (e.g., Aly, 1991; Hu et al., 2003). However, it is reminded here that whereas it is hard to construct a 3D force-free field with detached field lines interwound with simply connected field lines, the detached field in most analytical solutions is a self-closed flux rope in 2D. In the 3D reality, a twisted flux rope should be anchored to the solar surface, and the twisted field lines are simply connected again. It keeps an open question whether a really detached magnetic island in 3D, as that in the time-dependent MHD solutions of Gibson and Low (1998), is stable even if an analytical solution is available.

(2) The initial magnetic field is not force-free, i.e., the Lorenz force is balanced with gravity and pressure gradient. For example, it is estimated that the initial total energy would be increased by $10 \%$ if gravity is considered (Forbes, 2000), and the magnetic field with cross-field electric current may contain magnetic energy in excess of that in an open field (Low and Smith, 1993; Wolfson and Dlamini, 1997).

(3) Only a part of the initial magnetic field becomes open. Wolfson and Low (1992) demonstrated that the closed magnetic field in 2D may contain more energy than a partially open field with the same flux distribution at the solar surface, meaning that partial opening of the closed field through an ideal MHD process is allowable energetically, although the authors did not show how this proceeds. It was further pointed out by Low (1997) that the situation in 3D becomes

Living Reviews in Solar Physics

http://www. livingreviews.org/lrsp-2011-1 
much easier since an anchored flux rope in 3D can erupt by simply pushing its way amidst neighboring magnetic fields which can remain closed. As illustrated by Figure 35, Sturrock et al. (2001) quantified such a process by demonstrating that the initial magnetic energy of the closed field is larger than that of a partially open field, where only a part of the line-tied twisted field lines ruptures to the infinity. This indicates that such a rupture process is at least permissible from the energy point of view. With MHD numerical simulations, Fan (2005) reproduced such a rupture process in the early phase, while numerical resistivity led to magnetic reconnection in the current sheet formed below the erupting flux rope later. In a similar simulation, Inoue and Kusano (2006) inhibited magnetic reconnection by forcing the plasma velocity to be zero on and around the current sheet, and found that the flux rope can still erupt. With the zero-beta MHD simulations, Török and Kliem (2007) also claims that only torus instability enables the final eruption. More recently, Rachmeler et al. (2009) used a quasi-Lagrangian simulation technique to simulate the end state of the coronal field with different twists. Without magnetic reconnection in their simulations, they demonstrated that a twist flux rope, which is anchored to the solar surface at two ends, can rupture into a CME through an ideal MHD process. The key point is that some parts of the overlying arcade are pushed aside, leaving space for the erupting flux rope. It is noted that their current simulations are not based on a full MHD code, where mass and plasma dynamics are not included. Future efforts on MHD simulations without numerical resistivity are strongly encouraged to demonstrate that CMEs can erupt via ideal MHD processes.

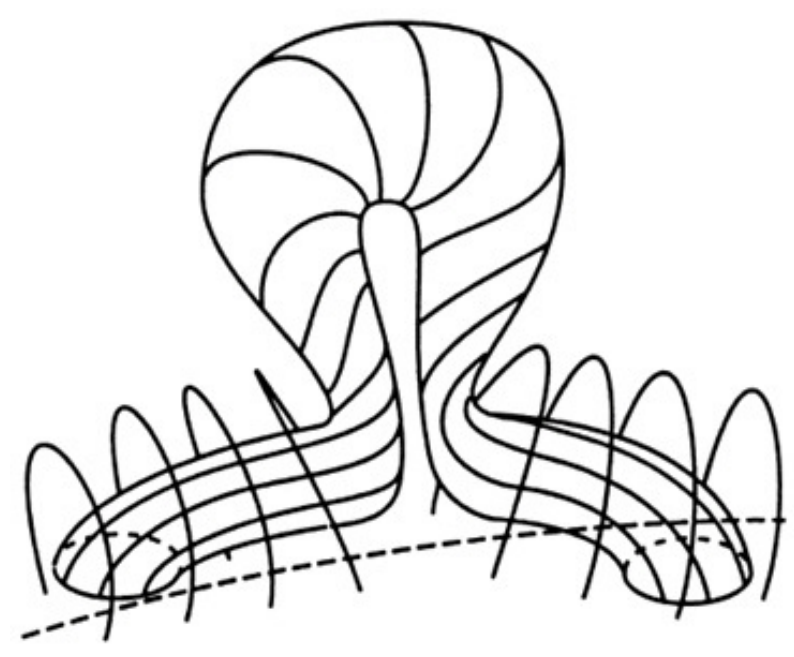

Figure 35: Schematic sketch showing that in the 3D space a twisted flux rope can rupture the overlying magnetic arcade and erupt alone by pushing the magnetic arcade aside (from Sturrock et al., 2001).

The significance of the above discussions is that the ideal MHD process is possible to release magnetic energy powering CMEs from a theoretical point of view. The observations, on the other hand, indicate that the majority of CMEs, especially those fast events, are strongly associated with solar flares, which result from magnetic reconnection (McAllister and Martin, 2000, see also Section 2 of this paper). In a unified model, Shibata (2003) emphasized that magnetic reconnection plays a crucial role in eruptions from small-scale jets to large-scale CMEs. Magnetic reconnection is also a key ingredient in the standard model for CME/flares, i.e., the so-called CSHKP model. The analytical modeling of this model in $2 \mathrm{D}$ indicates that the reconnection rate should be larger than a threshold in order for the flux rope to erupt into infinity, although the threshold is as small as $5 \times 10^{-3}$ (Lin and Forbes, 2000; Lin, 2002). This conclusion is different from that in Rachmeler et al. (2009), and the possible reason is that in the $2 \mathrm{D}$ case, all the overlying field lines above 
the flux rope would be stretched up as the flux rope erupts, which imposes stronger and stronger magnetic tension force hindering the flux rope from further eruption; whereas in the 3D case, some of the overlying magnetic loops can slip away from the erupting flux rope, as seen from Figure 35.

It is worth pointing out that although the resistivity of the coronal plasma is extremely low, it is not zero anyway, i.e., ideal MHD is just a theoretical model. As the closed structure erupts due to loss of equilibrium or instabilities via ideal MHD processes, current sheet formation is inevitable, where microscopic instabilities may excite anomalous resistivity or other non-ideal terms, and further launch magnetic reconnection. Therefore, the essence of the debate on the necessity of magnetic reconnection is to clarify whether reconnection plays a crucial role or a minor role in CME eruptions. It might be safe to say that magnetic reconnection plays an important role in most CMEs, especially the fast events which are of interest to space weather research. This is why the CME acceleration was found to be temporally correlated with the magnetic reconnection rate (Qiu et al., 2004). On the other hand, some other effects can also facilitate the eruption of the CMEs, e.g., ideal MHD instabilities, mass drainage, gas pressure, solar wind, etc. Especially, the higher corona, say, at $10^{5} \mathrm{~km}$ above the surface, is often not force-free (Gary, 2001), making these factors more effective. This explains why there is only a weak correlation between CME velocities and the peak SXR flux of the associated flare (Hundhausen et al., 1994; Yashiro et al., 2002; Vršnak et al., 2005; Yeh et al., 2005). As indirect evidence, there are some CMEs with proton flares, which were associated with a relatively weak impulsive phase of the flare (Cliver et al., 1983), and the filament eruption on 1981 December 5 generated an intense solar energetic particle (SEP) event and interplanetary type II radio bursts, but lacking an impulsive microwave burst (Kahler et al., 1986). All these indicate that some CMEs may be mainly accelerated by processes other than magnetic reconnection, which requires further confirmation.

When judging whether magnetic reconnection is involved in CME eruptions, the mostly used evidence is the SXR or EUV brightenings, especially solar flares, in the low corona. A ray-like structure under the some CMEs was also considered to be the direct evidence of a current sheet (Ciaravella et al., 2002, and followers). The width of such an assumed current sheet was estimated to be $\sim 10^{4} \mathrm{~km}$, and the corresponding resistivity was estimated to be 7 orders of magnitude larger than the anomalous resistivity (or 12 orders larger than the classical one) (Lin et al., 2007). It still awaits to be assured whether such a ray-like structure under some CMEs is really the current sheet where magnetic reconnection happens or it is actually the outward reconnection jet.

\subsection{Should fast and slow CMEs be attributed to different models?}

As mentioned in Section 2, CMEs are often associated with solar flares and/or filament eruptions. By studying 11 flares-associated CME events and 16 prominence-associated events observed by the white-light coronagraph on Skylab satellite, Gosling et al. (1976) found that these events are concentrated in two regimes, i.e., fast and slow CMEs as shown in Figure 36. Their analysis indicates that the faster events were almost always associated with flares and type II or IV metric radio bursts, whereas the slow events were associated with eruptive prominences. Such a result provoked a theoretical question on what determines the CME velocity.

Several years later, MacQueen and Fisher (1983) analyzed the speed-height plots of 12 looplike CMEs over the range $1.2-2.4 R_{\odot}$ observed by the MK3 coronagraph at the Mauna Loa Solar Observatory, and proposed the concept of two types of CMEs, i.e., flare-associated events that exhibit higher speeds (and show little acceleration with height) and filament eruption-associated events that exhibit slower speeds (and show large accelerations up to $50 \mathrm{~m} \mathrm{~s}^{-2}$ ). Sheeley Jr et al. (1999) used a new method to construct height-time maps of CMEs that were observed by SOHO/LASCO coronagraph. Similarly they classified CMEs into two types:

(1) Gradual CMEs: which are apparently formed when prominences and their cavities rise up from below coronal streamers, with speeds in the range $400-600 \mathrm{~km} \mathrm{~s}^{-1}$. They are often

Living Reviews in Solar Physics

http://www. livingreviews.org/lrsp-2011-1 


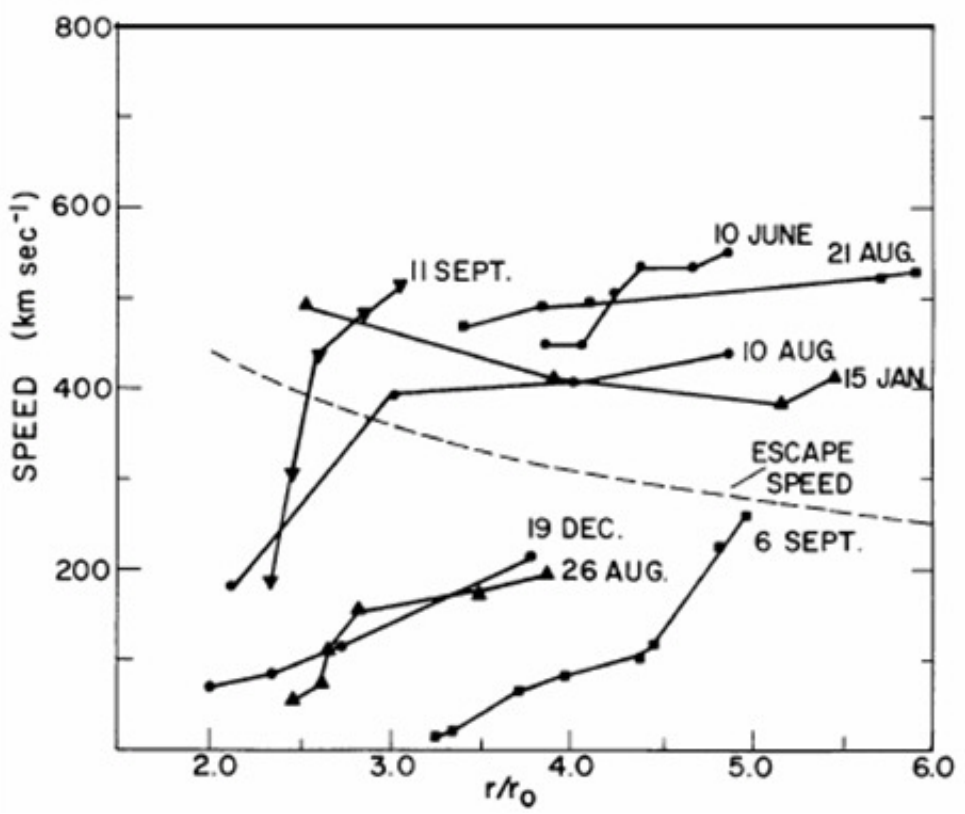

Figure 36: Velocity distributions along the height for some CMEs observed by Skylab satellite (Gosling et al., 1976).

accelerating in the field of view of the coronagraphs;

(2) Impulsive CMEs: which are often associated with flares and Moreton waves on the visible disk, with speeds typically in excess of $750 \mathrm{~km} \mathrm{~s}^{-1}$. They are often decelerating in the field of view of the coronagraphs.

Although Cane et al. (1986) had already pointed out that quiescent filament eruptions are not necessarily associated with slow CMEs, the concept of two types of CMEs in association with flares or filaments was widely recognized (St Cyr et al., 1999; Andrews and Howard, 2001). For instance, Moon et al. (2002) performed a statistical study of 3217 CMEs observed by SOHO/LASCO during 1996-2000, and found that the fraction of flare-associated CMEs has a tendency to increase with the CME speed, whereas the fraction of eruptive-filament-associated CMEs tends to decrease with the CME speed. They claim that such a result supports the classification of the two types of CMEs, although the accelerations of the two types of CMEs are both concentrated near 0 .

In order to explain the different kinematics, Low and Zhang (2002) phenomenologically proposed an idea for the two types of CMEs, i.e., the normal polarity flux rope eruptions correspond to the fast CMEs, whereas the inverse polarity flux rope eruptions correspond to the slow CMEs, as shown by Figure 37. On the other hand, it was found that, even for the inverse type only, the CME speed can be high or low (Chen and Krall, 2003; Wu et al., 2004). Whether the CMEs of the inverse polarity type would be statistically slower than the other type requires further observational and simulational support.

However, recently, Vršnak et al. (2005) presented a statistical analysis of 545 flare-associated CMEs and 104 non-flare CMEs in the heliocentric distance range of $2-30 R_{\odot}$. They found that there is no distinct difference between filament-associated CMEs and flare-associated CMEs. A similar conclusion was drawn by Chen et al. (2005a). With a sample of $4315 \mathrm{CME}$ events, Yurchyshyn et al. (2005) also found that there was no evidence to support the claim on two distinct types of CMEs. More and more evidence seems to indicate that the physics in the two types of CMEs is the same, with only the evolution time scale changing for individual events (Cliver and Hudson, 

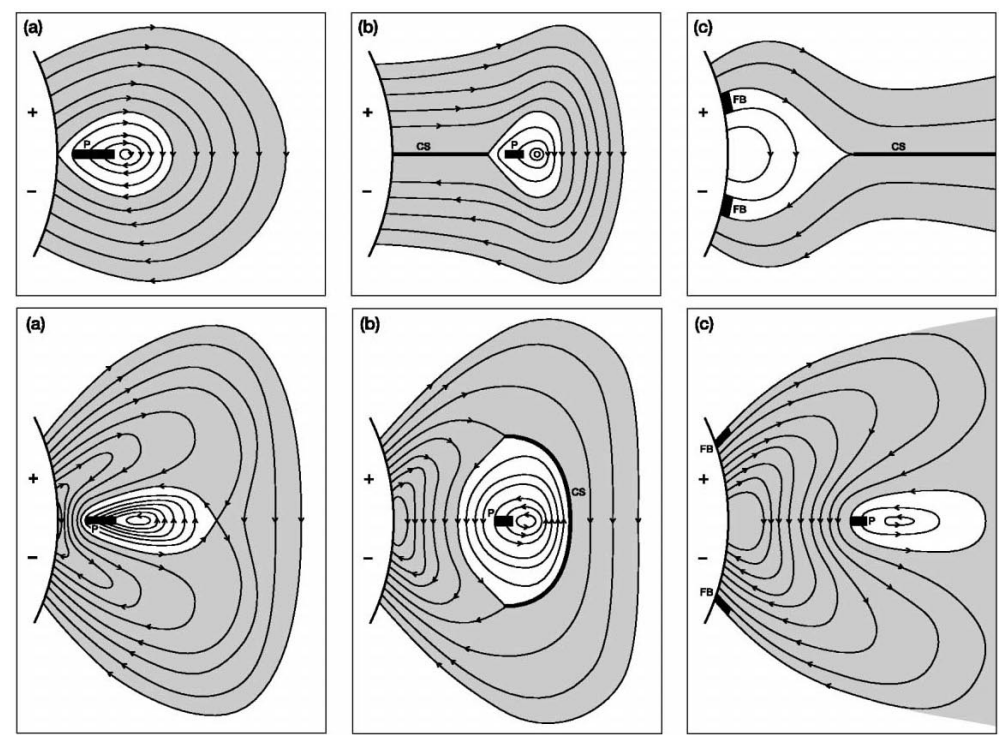

Figure 37: Phenomenological models for the slow CMEs (top panels) and the fast CMEs (bottom panels), where the filament is supported in an inverse polarity flux rope and a normal polarity flux rope, respectively (from Low and Zhang, 2002).

2002), i.e., for the faster CMEs, their characteristic time scales, e.g., the Alfvén transit time, are shorter, so the CMEs reach a high speed more rapidly.

The validation of the traditional classification of CMEs was further questioned by Feynman and Ruzmaikin (2004), who presented a CME associated with both a solar flare and an erupting filament. The analysis of this event and others led them to suggest that the apparent differences separating the two types of CMEs may be an observational effect, and all CMEs can be described by a single process as presented in Zhang et al. (2001a); see also Kahler et al. (1988) and Chen and Shibata (2000). Moreover, Chen et al. (2006a) pointed out that the traditional two-type classification, flare or filament associated, is logically incomplete since quite a lot of filament eruptions are accompanied by two-ribbon flares (e.g., Munro et al. 1979; see Zhang et al. 2001b for a typical event), which actually is the observational basis of the classical CSHKP model for $\mathrm{CME} /$ flares. Therefore, a complete classification should include the intermediate type of CMEs that are associated with both filament eruptions and flares. Therefore, they divide the events into three types in order to re-examine the CME velocity distributions: (1) CMEs associated with filament eruptions solely; (2) CMEs associated with flares solely; and (3) CMEs associated with both filament eruptions and solar flares. It was found that the average speeds of the three types of CMEs are $526 \mathrm{~km} \mathrm{~s}^{-1}, 564 \mathrm{~km} \mathrm{~s}^{-1}$, and $738 \mathrm{~km} \mathrm{~s}^{-1}$, respectively. The Kolmogorov-Smirnov test indicates that the $P$-value for the likelihood between the velocity distributions of these three types is very high, suggesting that there is no significant difference between these types of CMEs.

Several factors contributed to reaching the conclusion of the existence of flare-associated and filament-associated CMEs by early researches. The first, as pointed out by Vršnak et al. (2005), is that the samples used by them were too small to be statistically reliable. The second factor is that some prominences are rooted behind the solar limb. For these events, the low-lying flares can not be observed. Another factor is that some CMEs are associated with SXR giant arcades (Hiei et al., 1993), which are physically the same as solar flares but can not be detected by GOES satellites (Shibata, 1996). Hence, the giant arcade-associated events are often categorized into the non-flare CMEs. For instance, the 1997 January 6 CME event, which was widely claimed to be 
not associated with any flare, was shown to correspond to a tiny flare with the GOES classification slightly below A-class (Wu et al., 2002).

Even though, CMEs do show diverse velocities, which still provokes an important question: what factors determine the CME velocity? By analyzing 13 events, Qiu and Yurchyshyn (2005) found that the CME velocity is proportional to the total reconnection flux, whereas Chen et al. (2006a) found that the CME velocity is better correlated to the average strength of the magnetic field in the filament channel. These results are roughly consistent with the classical CSHKP model, where magnetic reconnection is supposed to occur below a filament or flux rope. The Lorentz force of the reconnected field lines accelerates the reconnection outflow to the Alfvén speed at the inflow region. The upward reconnection outflow pushes the filament or flux rope to erupt. The erupting velocity of the flux rope might be proportional to the reconnection outflow speed. Since the Alfvén speed is proportional to the magnetic field strength, it is not surprising that the CME velocity is roughly linearly proportional to the magnetic field strength. While, in both papers, the strong correlation is based on small samples, where reconnection probably plays the main role in accelerating these CMEs.

If the CME dynamics is mainly determined by magnetic reconnection, we might see a remarkable correlation between CME velocity and the intensity of the flare, and the CME velocity is mainly determined by the magnetic field strength of the source region. However, Hundhausen (1997), Yashiro et al. (2002), and Vršnak et al. (2005) revealed that there is only a weak correlation between the CME apparent velocity and the peak flux of the associated flares. The situation becomes even worse if the CME velocities are corrected for the projection effects (Yeh et al., 2005). This implies that some factors, in addition to reconnection, may affect the CME velocity. One factor is the filament and its material drainage. As the evidence for this, if filament-associated CME events are excluded, the relation between the CME speed and the flare intensity is significantly increased (Chen and Zong, 2009). Another major factor might be the restraining force of the background field overlying the CME, as proposed by Török and Kliem (2007), who found that a steep decay of the background field with height leads to a fast CME and a gentle decay leads to a slow CME.

\subsection{Nature and the driving source of "EIT waves"}

EIT waves were originally discovered by the EIT telescope on board the SOHO satellite as propagating wave-like fronts, with an emission enhancement ranging from $25 \%$ to less than $14 \%$, which is followed immediately by expanding EIT dimmings (Moses et al., 1997; Thompson et al., 1998). as depicted in the base-difference images of Figure 38. They can be observed simultaneously at various EUV wavelengths, such as $171 \AA$ (formation temperature $1 \mathrm{MK}$ ), $195 \AA$ (formation temperature 1.4 MK), and $284 \AA$ (formation temperature 1.9 MK) (Wills-Davey and Thompson, 1999; Zhukov and Auchère, 2004; Long et al., 2008), suggesting that the brightening is mainly due to density enhancement. Their propagation velocity ranges typically from 170 to $350 \mathrm{~km} \mathrm{~s}^{-1}$ in the events observed by the SOHO/EIT telescope (Klassen et al., 2000; Thompson and Myers, 2009). With higher time cadence, the Extreme Ultra Violet Imager (EUVI) on board the STEREO satellite showed that the EIT wave velocity can be as small as $\sim 10 \mathrm{~km} \mathrm{~s}^{-1}$ and as high as $475 \mathrm{~km} \mathrm{~s}^{-1}$ (Long et al., 2008; Zhukov et al., 2009). When the large-scale magnetic configuration is simple, for instance, with only one active region on the visible disk, the EIT wave fronts are almost circular (Thompson et al., 1998). However, when there are other active regions surrounding the source region of the eruption, the EIT waves appear in patches, managing their ways outward while avoiding active regions (Thompson et al., 1999). It is found that they stop near the boundary of coronal holes (Thompson et al., 1999).

The discovery of such an unexpected and spectacular wave sparked a lot of interests as well as debates among the community (see Chen, 2008; Wills-Davey and Attrill, 2009; Warmuth, 2010; Gallagher and Long, 2010, for reviews). The modelings of EIT waves are important in the sense 

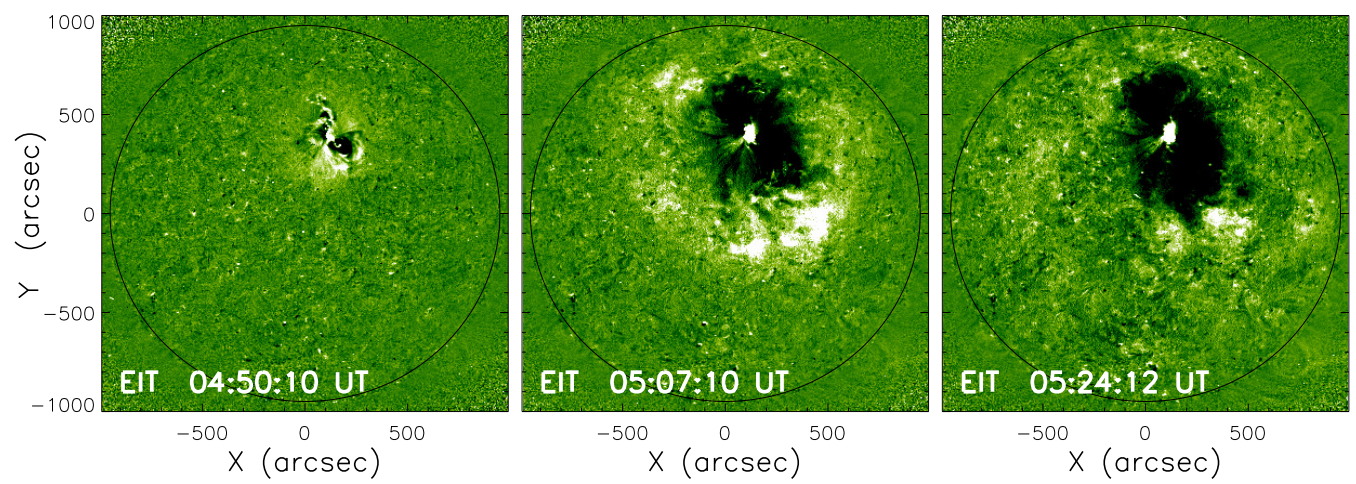

Figure 38: EIT $195 \AA$ base difference images showing the evolution of the most famous EIT wave event on 1997 May 12 (from the SOHO/EIT data archive).

that: (1) they may provide crucial clues for the understanding of CMEs (Chen, 2009a); (2) they may be used to diagnose the coronal magnetic topology (Attrill et al., 2006; Zhang et al., 2007); and (3) they can be used in coronal seismology to diagnose the magnetic field (Warmuth and Mann, 2005; Ballai, 2007; Chen, 2009b). Several models have been proposed so far, and the debates are mainly focused on two issues: (1) What drives the EIT waves: flare or CME? (2) What is the nature of EIT waves? While there is a converging consensus that EIT waves are physically linked to CMEs rather than flares (Biesecker et al., 2002; Cliver et al., 2005; Chen, 2006), the nature of EIT waves is still under hot debate. Here we briefly introduce several models, and more details can be found in the reviews by Wills-Davey and Attrill (2009) and Gallagher and Long (2010).

\subsubsection{Fast-mode MHD wave model}

Since "EIT waves" propagate across the magnetic field lines, with a velocity sometimes larger than the sound speed in the corona, and are associated with density enhancement, they are unlikely to be slow-mode MHD waves or Alfvén waves. Therefore, they were immediately considered to be fast-mode magnetoacoustic waves in the corona (e.g., Wang, 2000; Wu et al., 2001; Vršnak et al., 2002; Warmuth et al., 2004; Grechnev et al., 2008; Pomoell et al., 2008; Temmer et al., 2009; Schmidt and Ofman, 2010), being regarded as the coronal counterpart of the $\mathrm{H} \alpha$ Moreton waves (Moreton and Ramsey, 1960; Uchida, 1968). Figure 39 shows the observation of the EIT wave event on 1997 May 12 (upper panels) and the MHD simulation result of the fast-mode wave (lower panels), which are claimed to match very well (Wu et al., 2001). Several groups came to the same conclusion from the observational point of view (e.g., Veronig et al., 2008; Patsourakos et al., 2009; Kienreich et al., 2009; Veronig et al., 2010).

However, it is difficult fot the fast-mode wave model to explain the following features of "EIT waves" (e.g., see Wills-Davey et al., 2007; Chen, 2008, for details): (1) the "EIT wave" velocity is significantly smaller than those of Moreton waves. The latter are generally believed to be due to fast-mode waves in the corona (Uchida, 1968; Eto et al., 2002); (2) The velocities of "EIT wave" have no any correlation with those of type II radio bursts (Klassen et al., 2000). The latter are also well established to be due to fast-mode shock waves in the corona; (3) The "EIT wave" fronts may stop when they meet with magnetic separatrices (Delannée and Aulanier, 1999; Delannée et al., 2007; Delannée, 2009); (4) The "EIT wave" velocity may be below $100 \mathrm{~km} \mathrm{~s}^{-1}$ (e.g., the Figure 3 of Long et al., 2008; Zhukov et al., 2009), which is even smaller than the sound speed in the corona. Some of these strange features provoked Delannée and Aulanier (1999) and Delannée (2000) to question the fast-mode wave model for the first time.

Living Reviews in Solar Physics

http://www . livingreviews . org/lrsp-2011-1 

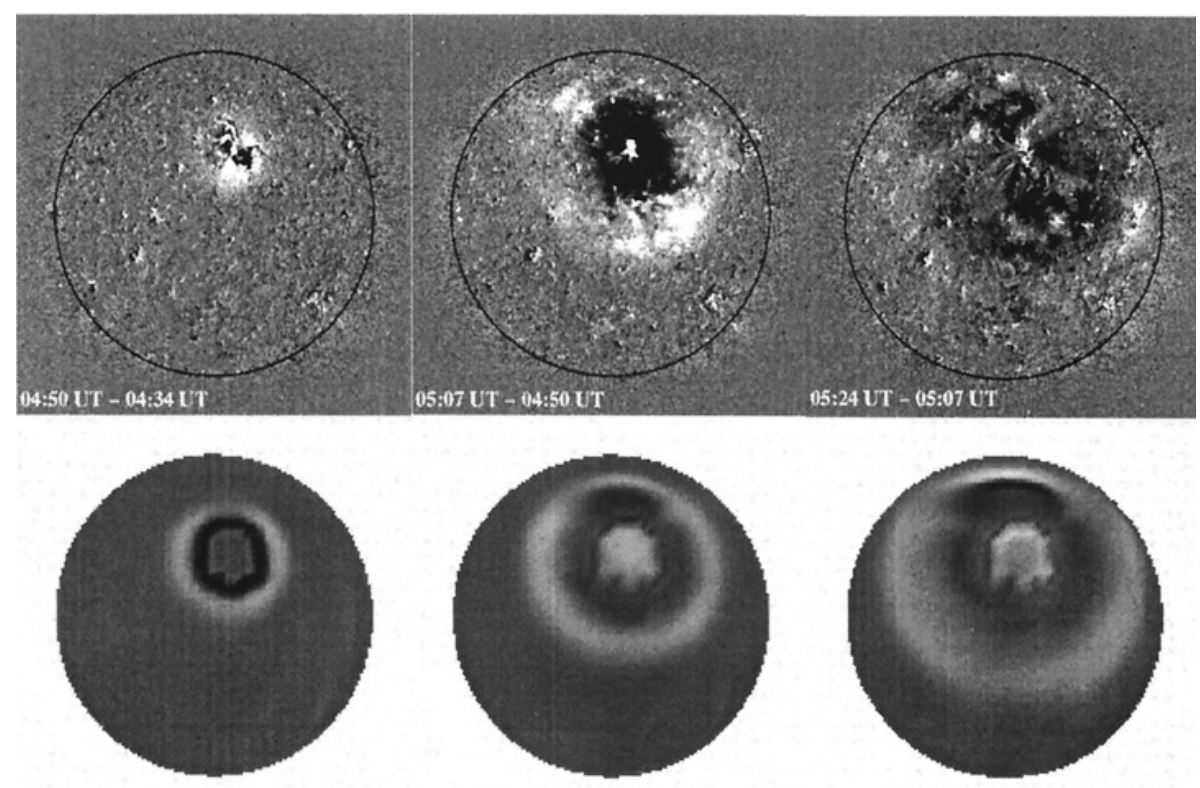

Figure 39: Comparison between the observation of the 1997 May 12 EIT wave event and the 3D MHD simulation of fast-mode waves in the low corona (adapted from Wu et al., 2001).

\subsubsection{Magnetic fieldline successive stretching model}

In 2D MHD numerical simulations, Chen et al. (2002; 2005b; 2005c; 2006b) identified the "EIT wave" to be the density-enhanced boundary of the dimming region (the dimming is indicated by the blue region in the upper panels of Figure 40), which is located well behind the fast-mode piston-driven shock waves (the red front in the upper panels of Figure 40) during CME eruptions. They proposed that "EIT waves" are apparently-moving density enhancements, which are actually produced by successive stretching of the closed field lines overlying the erupting flux rope, rather than being real waves. The model is illustrated in the bottom panels of Figure 40: as the flux rope erupts, the overlying field lines will be pushed to stretch up successively by the erupting flux rope, and for each field line, the stretching starts from the top, and is then transferred to the footpoints, where the stretched field line compresses the plasma to form a bright front. That is to say, the stretching starts from point $\mathrm{A}$ and then propagates to point $\mathrm{C}$ with the local fast-mode wave speed. At the same time, the stretching propagates from point $\mathrm{A}$ to point $\mathrm{B}$ and then to point $\mathrm{D}$ with the local fast-mode wave speed. Therefore, the apparent speed for the EIT wave to propagate from point C to point $\mathrm{D}$ is $v_{\mathrm{EIT}}=C D / \Delta t$, with $\Delta t=\int_{A}^{B} 1 / v_{f} d s+\int_{B}^{D} 1 / v_{A} d s-\int_{A}^{C} 1 / v_{A} d s$, where $v_{A}$ is the Alfvén speed, and $v_{f}$ is the fast-mode wave speed perpendicular to the field line, and the last two integrals are along the field line shown in Figure 40 (see Chen et al., 2002, 2005c, for details). If the field lines are semi-circles, $v_{\text {EIT }}$ is found to be about one-third of the fast-mode wave speed. When the EIT wave encounters another active region (to the right of point D in Figure 40) or an open field (in both cases there is a magnetic separatrix), no stretching can be transferred into the closed region or the open field, and the EIT wave stops near the magnetic separatrix, as demonstrated by Chen et al. (2005c). The model can account for the main characteristics of "EIT waves", such as their low velocity, their diffuse fronts, and the stationarity near magnetic separatrices. The model also predicts that there should be two EUV waves, one is the coronal counterpart of Moreton wave, which has a sharp front, and the other is the diffuse "EIT wave". The model found support in observations (e.g., Harra and Sterling, 2003) and in 3D MHD simulations (Downs et al., 2011). 

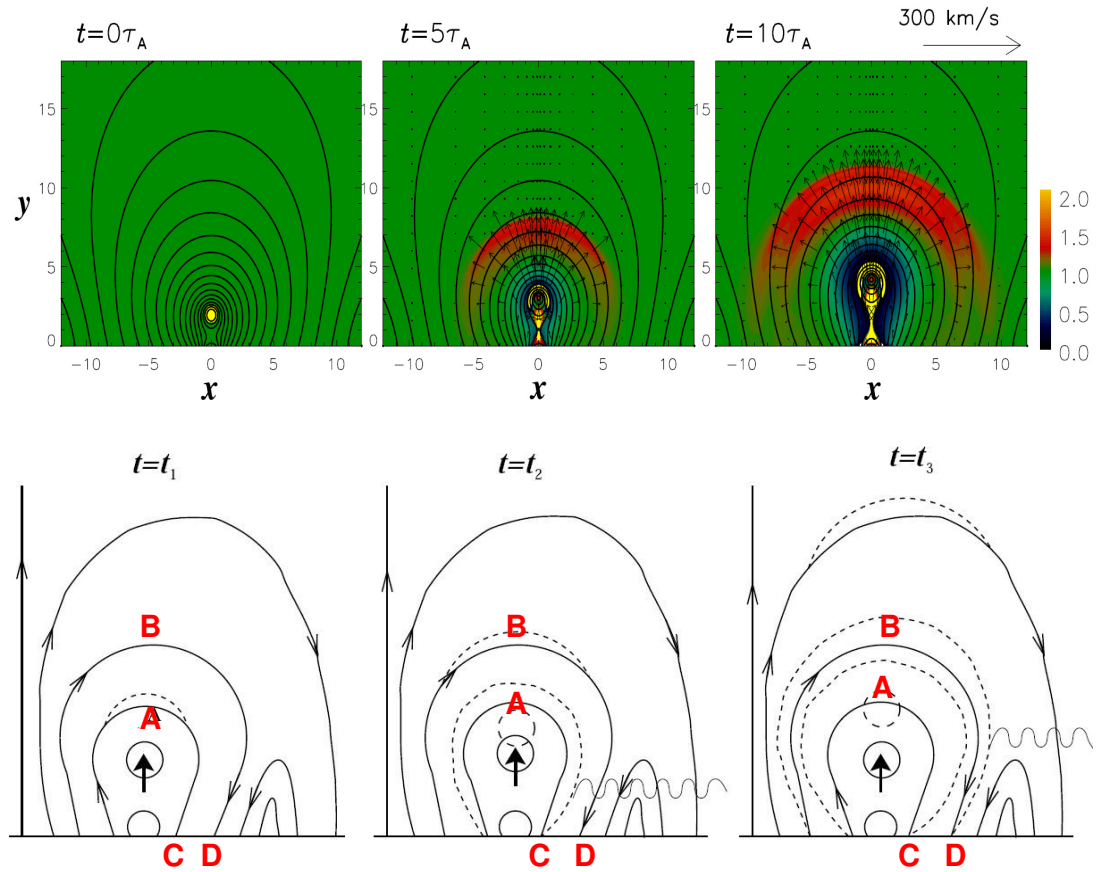

Figure 40: Upper panels: The evolutions of the density (color, in unit of $1.67 \times 10^{-12} \mathrm{~kg} \mathrm{~m}^{-3}$ ), magnetic field (lines), and velocity field (arrows) as a flux rope is ejected upward. The scale for the velocity is shown at the upper-right corner. Lower panels: the schematic sketch of the fieldline successive stretching model for EIT waves (adapted from Chen et al., 2002).

\subsubsection{Successive reconnection model}

Noticing that the EIT wave fronts rotate apparently in the same direction as the erupting filament, Attrill et al. (2007) also claimed that EIT waves should be related to the magnetic rearrangement. As shown in Figure 41, they proposed a successive reconnection model, i.e., EIT wave fronts are the footprint of the CME frontal loop, which is formed due to successive magnetic reconnection between the expanding core field lines and the small-scale opposite polarity loops.

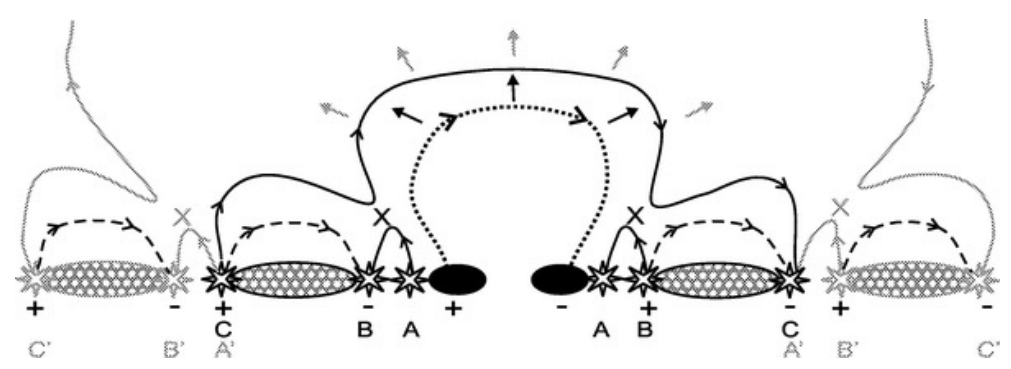

Figure 41: The schematic sketch of the successive magnetic reconnection model for EIT waves (from Attrill et al., 2007).

The successive reconnection model was criticized by Delannée (2009), who demonstrated that the extrapolated potential field for the famous EIT wave event on 1997 May 12 does not fix into the model illustrated in Figure 41. The discrepancy can be reconciled if we accept that reconnection can happen occasionally during the CME expansion, but not always. This is consistent with the recent 
3D MHD simulations by Cohen et al. (2009). They proposed that along with the CME eruption the "EIT wave" front consists of two wave components. The bright non-wave component is produced by the CME expansion (or called field line stretching in Chen et al., 2002), which is facilitated by magnetic reconnection (Attrill et al., 2007). The weak wave component is initially attached to the bright component, and then becomes detached after the CME ceases lateral expansion.

\subsubsection{Current shell model}

Through 3D MHD simulations, Delannée et al. (2008) found that as a flux tube erupts, an electric current shell is formed by the return currents of the system, which separate the twisted flux tube from the surrounding fields, as shown in Figure 42. Slightly different from their early idea of magnetic rearrangement (Delannée and Aulanier, 1999), they claim that this current shell corresponds to the "EIT waves". They also revealed that the current shell rotates, similar to the apparent rotation of the EIT wave fronts found by Podladchikova and Berghmans (2005). They also emphasized the role of Joule heating in the current shell in explaining the EIT wave brightening, which was criticized by Wills-Davey and Attrill (2009).
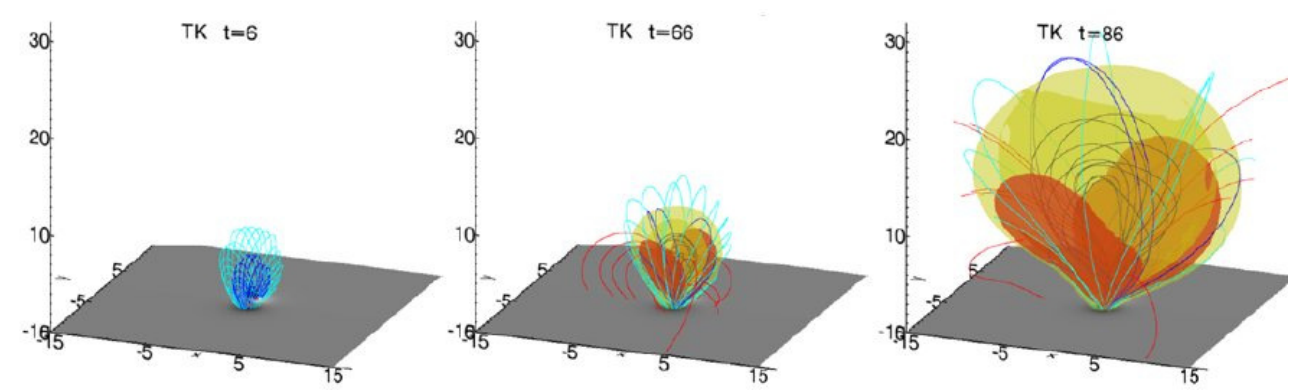

Figure 42: The evolutions of the magnetic field (lines) and $\mathbf{J} \cdot \mathbf{B} / B^{2}$ (isosurfaces) in the MHD simulations of Delannée et al. (2008). The isosurface of $\mathbf{J} \cdot \mathbf{B} / B^{2}$ indicates a current shell just outside the erupting flux tube (adapted from Delannée et al., 2008).

\subsubsection{Slow-mode wave or soliton wave model}

Noticing that EIT waves generally keep single-pulse fronts and that the EIT wave velocity is sometime smaller than the sound speed in the corona, Wills-Davey et al. (2007) speculated that the EIT waves might be best explained as a soliton-like phenomenon, say, a slow-mode solitary wave. They stated that a solitary wave model can also explain other properties of the EIT waves, such as their stable morphology, the nonlinearity of their density perturbations, the lack of a single representative velocity, and their independence of Moreton waves. There is no modeling of such an idea.

Wang et al. (2009) performed 2D MHD numerical simulations of a flux rope eruption, where they found that behind the piston-driven shock appear velocity vortices and slow-mode shock waves. They interpret the vortices and the slow-mode shock wave as the EIT waves, which are $40 \%$ as fast as the Moreton waves. 


\subsection{What is the nature of CMEs?}

We are coming back to the most fundamental question: What is the CME? This question may come up as a surprise to many colleagues since it is often taken for granted that CMEs are coronal mass ejected into the interplanetary space, without thinking too much about what the CMEs really are.

There is a general consensus about the CME core, which can be easily identified to be the erupting filament/prominence based on their kinematics from near the solar limb to high corona (e.g., House et al., 1981; Low, 1990; Gilbert et al., 2000). The only possible correction might be that the upward reconnection outflow, as shown in Figure 34, would keep refilling the CME core in the form of hot plasmas (e.g., Ohyama and Shibata, 2008). However, as for the CME frontal loop, the understanding is rather illusive.

The CME frontal loop, or leading edge, was initially regarded as a wave phenomenon (Nakagawa et al., 1975; Steinolfson and Nakagawa, 1977), i.e., they are fast-mode MHD waves excited by the pressure pulse from the underlying solar flare. This theory was later discarded since many observations, e.g., Howard et al. (1982); Fisher and Munro (1984); and St Cyr et al. (2000), indicate that at several solar radii, the two legs of the CME frontal loop do not expand laterally. An improved model was proposed by Wu et al. (1983), where the MHD waves were produced by moving mass as suggested by the SXR observations.

The second popular model is that the CME frontal loop is a bundle of the background coronal magnetic field lines filled with plasmas (e.g., Poland and Munro, 1976). In particular, it was proposed that the three-part structure of the CME, i.e., the frontal loop, the cavity, and the core, can be identified as the dome, the cavity, and the prominence of a helmet streamer that transits drastically into a mass motion (Low, 1984, 1990; Hundhausen et al., 1984). For example, Gibson et al. (2006b) reported a pre-existing structure (consisting of a cavity and a bright loop outside of it) erupts, producing a typical three-part CME. Similarly, it was proposed that the CME frontal loop could be a shell-like layer draped over the flux rope (e.g., Chen, 1996; Krall et al., 2001; Thernisien et al., 2009), which is roughly perpendicular to the flux rope. In particular, Chen et al. (2000) and Krall and Chen (2005) suggested that the CME bright rim is ahead of the flux rope axis by $2 a$, where $a$ is the minor radius of the flux rope.

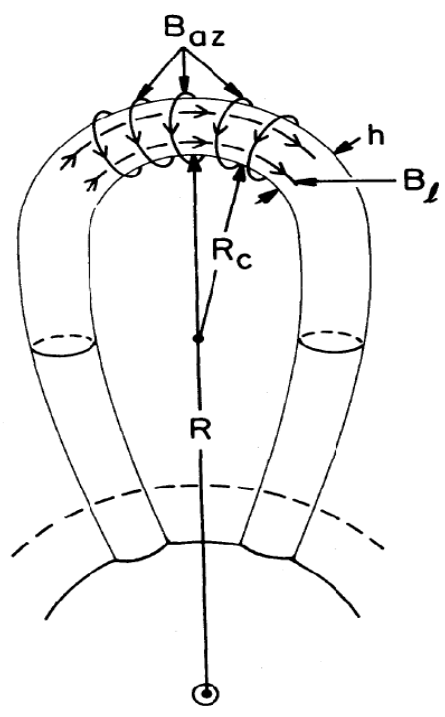

Figure 43: The flux rope model for the CME frontal loop (from Mouschovias and Poland, 1978). 
The third model is that the CME frontal loop is a twisted flux rope expanding and broadening in the background coronal plasma and magnetic field (Mouschovias and Poland, 1978), as shown in Figure 43.

One common feature implied by the above-mentioned two models is that the two legs of the CME frontal loop, whose plasma is frozen in the line-tied magnetic field lines, should be fixed near the solar surface. This might be in contradiction with the observational fact that CMEs expand laterally in the low corona (e.g., St Cyr et al., 1999). Another common feature implied by these two models is that the top part of the CME frontal loop moves radially as a mass motion. However, the spectroscopic observations with the Ultraviolet Coronagraph Spectrometer (UVCS) on board the SOHO satellite revealed that the Doppler velocity of the CME frontal loop is significantly smaller than its apparent propagation velocity, indicating that the frontal loop propagation is not a mass motion, at least for halo CMEs, as discussed in Ciaravella et al. (2006). They suggested that CME frontal loop may correspond to dense coronal plasmas swept up by a shock or compression wave. In the review paper of Forbes (2000), the CME frontal loop is considered to be plasma pile-up, while the detailed physical process of the piling-up needs to be clarified.

In the research aimed to investigate the relationship between CMEs and "EIT waves", Chen (2009a) proposed another idea on the formation of the CME frontal loop. He compared the whitelight coronagraph observation of the 1997 September 9 CME event and the EIT base-difference images, and found that the white-light CME frontal loop is cospatial with the EIT wave front. The cospatiality of "EIT waves" and CME leading loops, as confirmed by Dai et al. (2010), led Chen (2009a) to extend the magnetic fieldline stretching model for EIT waves (Chen et al., 2002, 2005c) to explain the formation of the CME frontal loop, which is described as follows. As illustrated by Figure 44, as the core structure, e.g., a magnetic flux rope, erupts, the resulting perturbation propagates outward in every direction, with a probability of forming a piston-driven shock as indicated by the pink lines. However, different from a pressure pulse, the erupting flux rope continues to push the overlying magnetic field lines to expand, so that the field lines are stretched outward one by one. For each field line, the stretching starts from the top, e.g., point A for the first magnetic line, and then is transferred down to the leg (point D) with the Alfvén speed, by which the first field line is stretched entirely. The deformation at point $\mathrm{A}$ is also transferred upward to point $\mathrm{B}$ of the second magnetic field line with the fast-mode wave speed. Such a deformation would also be transferred down to its leg (point E) with the local Alfvén speed, by which the entire second magnetic field line is stretched up. The stretching of the magnetic field lines compresses the coronal plasma on the outer side of the field line, producing density enhancements. All the newly formed density enhancements at a given time form a pattern (green), which is observed as the CME frontal loop. Similar to "EIT wave" fronts, the legs of the CME leading loop separate initially, and may stop when they meet with magnetic separatrices such as the boundary of coronal holes. This is why CMEs generally maintain a fixed angular span in their later stages. At the same time, as the field lines are stretched outward, the enveloped volume increases, resulting in coronal dimmings (or the dark cavity) behind the CME leading loop.

According to the above fieldline stretching mechanism (Chen et al., 2002, 2005c), both CME frontal loop and the EIT wave front are a dome-like structure, and the propagation of the CME frontal loop is an apparent motion, rather than a mass motion at this stage (the plasma is moving outward, but with a smaller velocity). It is due to the successive stretching of closed magnetic field. Near the footpoint, the lateral expansion speed is the same as that of EIT wave; Near the top part of the CME frontal loop, its radial propagation velocity is the local fast-mode wave speed, significantly larger than the mass motion velocity. Only when the CME propagates to a certain height where the local fast-mode wave speed decreases to the mass motion velocity of the plasma in the frontal loop, the radial expansion of the CME becomes a real mass motion. This model explains why some CMEs were observed to be very fast even in the very low corona (e.g., MacQueen and Fisher, 1983) and why these fast CMEs would decelerate during their propagation, i.e., the local 

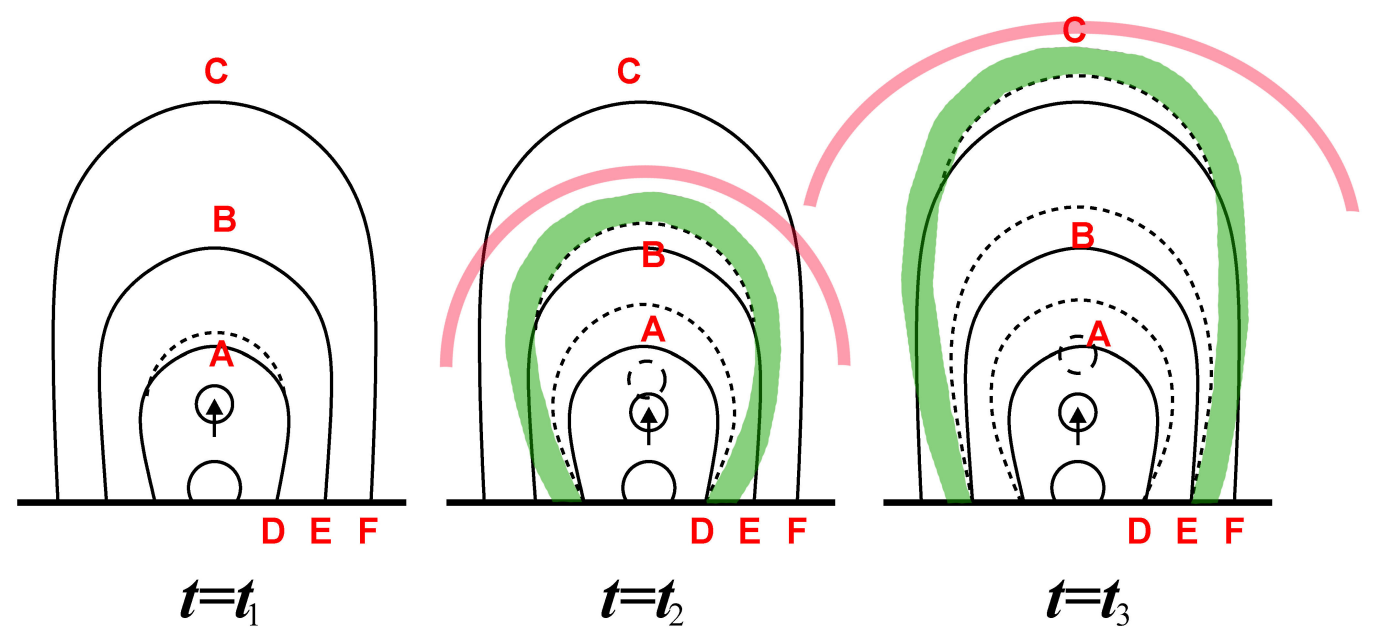

Figure 44: A schematic sketch of the formation mechanism of CME leading loops, where the CME leading loop (green) are apparently-moving density enhanced structure that is generated by the successive stretching of magnetic field lines as the erupting core structure, e.g., a flux rope, continues to push the overlying field lines to expand outward successively. The piston-driven shock is shown as pink lines (from Chen, 2009a).

fast-mode wave speed decreases with height.

However, Zhang et al. (2001a) did show a bright loop expands very slowly in the LASCO/C1 field of view, and then accelerates rapidly. Our conjecture is that the slowly-moving bright loop is actually a coronal loop straddling over a flux rope, rather than the CME frontal loop. As magnetic reconnection is excited below the flux rope, the flux rope is accelerated. The erupting flux rope stretches up the coronal loop as well as the overlying magnetic field lines successively, forming a new density-enhanced shell, i.e., the CME frontal loop, with the mechanism illustrated in Figure 44.

On the other hand, the SOHO/LASCO coronagraph also showed some slow CMEs which are accelerating gradually during their passage over several solar radii. For example, the 2002 May $16 \mathrm{CME}$ event accelerates from a velocity of $\sim 20 \mathrm{~km} \mathrm{~s}^{-1}$ at a height of $4 R_{\odot}$ to $279 \mathrm{~km} \mathrm{~s}^{-1}$ at $18 R_{\odot}$. CME events like this are strongly reminiscent of the expansion of coronal loops, especially the interconnecting loops, being dragged out by the ambient solar wind.

Here, I tentatively propose a unified paradigm for CME eruptions: as the CME progenitor is triggered to rise, it may erupt in one of the two ways:

(1) If there is weak or even no magnetic reconnection that can cut some of the line-tied magnetic field lines from the solar surface, the CME is accelerated slowly, probably due to ideal MHD instabilities or by the ambient solar wind. In this case, the CME frontal loop propagation is a mass motion, with the final velocity approaching that of the solar wind;

(2) If there is significant magnetic reconnection that can cut some of the line-tied magnetic field lines from the solar surface, the CME progenitor, e.g., a flux rope, is accelerated rapidly, stretching up the overlying field lines successively. Such a successive stretching compresses the coronal plasma, forming a new density-enhanced pattern, i.e., the CME frontal loop (see Bao et al., 2006, for the observation of the possible formation of a CME frontal loop). In this case, the propagation of the frontal loop is not a mass motion, and its top part moves outward at the local fast-mode wave speed. 


\subsection{Are halo CMEs special?}

Halo CMEs appear as an annulus surrounding the solar disk propagates outward. It is widely accepted that they are nothing but CMEs propagating near the Sun-Earth direction, either toward or away from the Earth (Howard et al., 1982). However, statistical investigations indicate that the average velocity of halo CMEs, $\sim 957 \mathrm{~km} \mathrm{~s}^{-1}$, is twice as large as that of normal CMEs (e.g., Yashiro et al., 2004), which seems to make halo CMEs special.

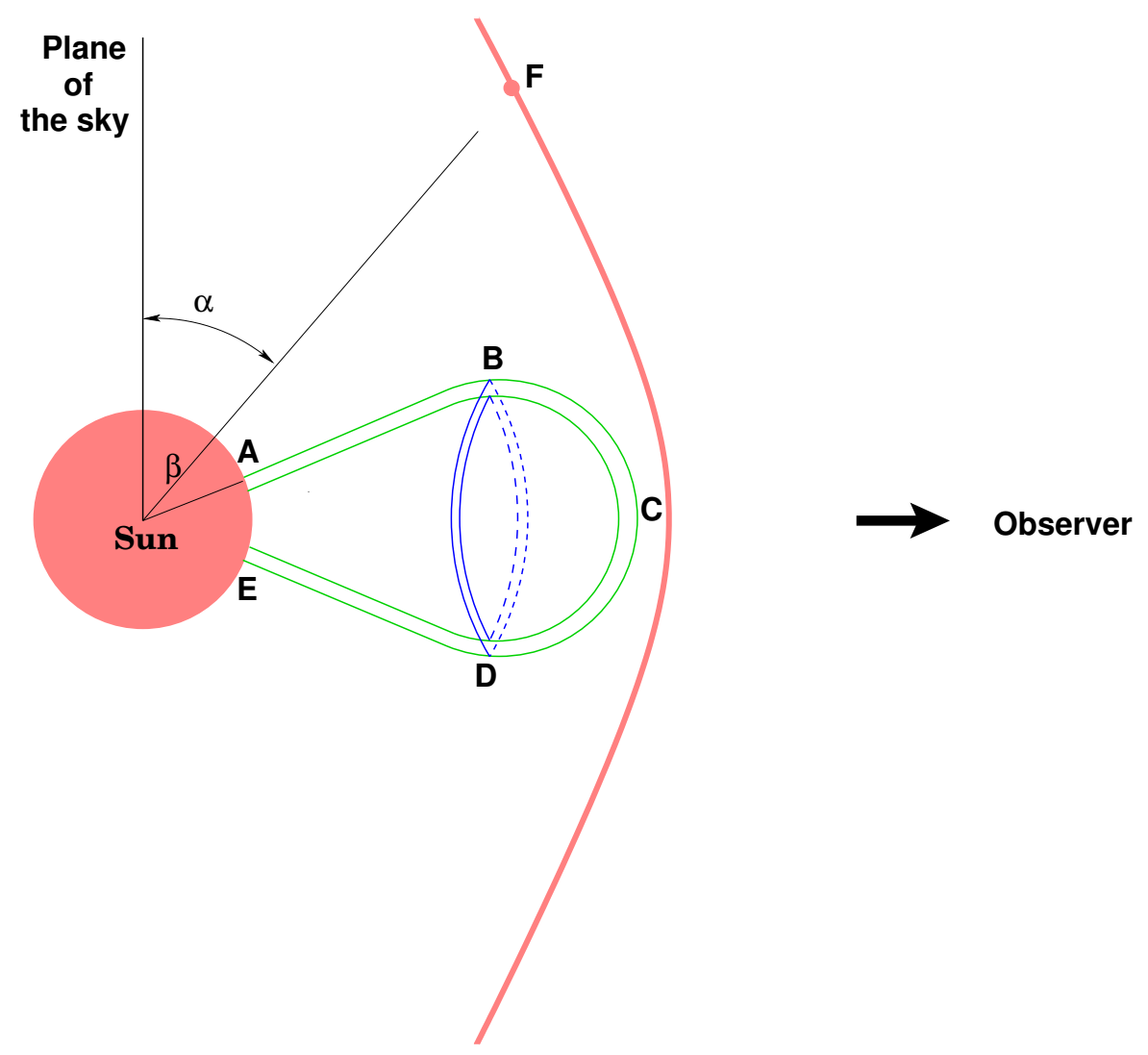

Figure 45: A sketch showing how a dome-like CME would be observed as a limb event (green lines) and as a halo event (blue lines). The pink line ahead of the CME is the fast-mode piston-driven shock wave. $\alpha$ is the inclined angle between the radial direction of point $\mathrm{F}$ and the plane of the sky, whereas $\beta$ is the angle between the CME leg and the plane of the sky. It is seen that the far wing, say, near point F, propagates in a direction closer to the plane of the sky than the CME does (since $\alpha<\beta$ ).

It may be natural to think that the nearly circular front of halo CMEs is just the face-on view of the dome-like CME frontal loop. For simplicity, we assume that the CME frontal loop is represented as the shell of a cone-shaped dome as illustrated by Figure 45. When it is observed edge-on as a limb event, it would appear as a loop structure, i.e., the green lines linking points A, B, C, D, and E, since the optical thickness is much larger here. When the CME is observed face-on as a halo event, however, the optical thickness is large only near the torus linking points B and D, i.e., the blue lines in Figure 45, which surrounds the solar disk to the observer. If so, the nature of halo CMEs would be the same as the normal CMEs. For example, Krall et al. (2006) extended the flux rope model, which was demonstrated to be applicable to limb CMEs (Chen, 1996; Krall et al., 2001), to halo CMEs, and found that the model can reproduce both quantitative 
near-Sun properties of the 2003 October $28 \mathrm{CME}$ and the timing, strength, and orientation of the fields measured in situ near the Earth orbit. Of course, the nature of CME frontal loops is still under debate, and there are other possibilities, such as that the CME frontal loop is due to the compression of magnetic field lines which are stretched successively, as discussed in Section 4.4.

If the nature of halo CME fronts is the same as normal CMEs, there is a serious problem: why halo CMEs are on average twice faster than normal CMEs? Noticing that the Thomson scattering is significantly reduced for halo CMEs, Andrews (2002) proposed that many dim and slow halo CMEs are missed by coronagraphs so that the average velocity of the observed halo CMEs is high. Following this line of thought, Zhang et al. (2010) performed Monte Carlo simulations to investigate how the white-light brightness of CMEs with an average velocity of $523 \mathrm{~km} \mathrm{~s}^{-1}$ is reduced when they are observed as halo events. They found that the brightness of many narrow and slow CMEs, when they are observed as full halo CMEs, is reduced to a level comparable to the solar wind fluctuations, and therefore, these events would be missed to be identified in the coronagraph images. The remaining observable halo CMEs have an average velocity of $\sim 922 \mathrm{~km} \mathrm{~s}^{-1}$, quite similar to the value in observations.

An alternative view is that the halo CME fronts are completely different from the frontal loops of limb CMEs in physics. For example, Lara et al. (2006) proposed that the halo CME fronts might be the combination of the CME-driven shock wave and the CME material itself. Based on MHD numerical simulations, Manchester IV et al. (2008) synthesized the white-light images of a halo CME event, and found that the halo CME front can be identified as the CME-driven shock wave.

Since the fast-mode wave in the corona is of the order of $1000 \mathrm{~km} \mathrm{~s}^{-1}$, it easily explains why the average velocity of halo CMEs is as high as $957 \mathrm{~km} \mathrm{~s}^{-1}$. One may argue that the piston-driven shock wave is much weaker in white light than the CME frontal loop, and therefore can be barely visible in the coronal images. This is true for the limb events (e.g., Vourlidas et al., 2003). However, for halo CMEs, as illustrated by Figure 45, the situation may change. If we consider the Thomson scattering, which remarkably favors the plasma moving in a direction closer to the plane of the sky, the scattered white-light emission of the shock wave front at point $\mathrm{F}$ in Figure 45 could be stronger than that of the CME frontal loop near point B when both points are observed at the same projected heliocentric distance in the plane of the sky, although the plasma density is higher at point $\mathrm{B}$ than point $\mathrm{F}$. Such a possibility needs to be studied quantitatively. Another question in this theoretical framework is how to interpret a couple of slow halo CME events, whose velocities are $\sim 100-200 \mathrm{~km} \mathrm{~s}^{-1}$. Maybe weak magnetic field? 


\section{Summary}

Since the discovery of CMEs in the early 1970s, the spectacular phenomenon has attracted wide interest, and has been extensively investigated through observations, theoretical analysis, and numerical simulations. The related research keeps being a hot topic in solar physics, not only because CMEs are associated with many other solar eruptive phenomena of different scales, but also because they are the major driving source of the hazardous space weather environments near the Earth. With the improving ground-based and space-borne observations along with the more and more sophisticated modelings, much progress has been obtained in the understanding of CMEs, yet with controversies in every aspect, including the progenitors, the triggering mechanisms, their propagation, the interaction between CMEs with the background solar wind, and in particular, how CMEs are related to other phenomena. The improving knowledge on these aspects directly determines how well we can predict the commencement of CMEs and their impact on the space weather environment. Here we summarize the key issues described in this review paper, with the hope that readers can grasp the general consensuses and controversies about the CME models, which might unavoidably be biased on the author's personal viewpoints. Detailed reviews on each issue are distributed in the main text.

Morphologically, CMEs can be distinguished as narrow and normal CMEs. The essential difference between them is not the angular width, since some normal CMEs also have a small angular width, e.g., $\sim 10^{\circ}$. The meaningful difference might be that "narrow CMEs" are jet-like, whereas normal CMEs are loop-like. The physics of narrow CMEs is quite clear, i.e., they correspond to the outflow as emerging flux or a coronal loop reconnects with the open magnetic field. Therefore, the contents hereafter refer to the normal CMEs.

(1) Classification: Kinematically, the apparent CME velocity covers a wide range from tens to more than $3000 \mathrm{~km} \mathrm{~s}^{-1}$. Some are very fast, whereas some are very slow. It was once proposed that they can be divided into impulsive CMEs that are associated with solar flares and gradual CMEs that are associated with erupting prominences. Later observations tend to discard such a classification in the sense that there is no a dividing line between the velocity distributions of these two types of CMEs and many CMEs are associated with both solar flares and prominence eruptions.

(2) Progenitors: The pre-CME structure might be strongly sheared or weakly twisted magnetic field (i.e., core field) that is restrained by less-sheared envelope field, and a flux rope is not necessarily required in the progenitor. Filaments and sigmoids might correspond to the inner and outer parts of the core field, respectively. Whether a closed magnetic structure can erupt to form a CME depends not only on how sheared the core field is, but also on how fast the envelope field decreases with height. These two factors need to be combined and investigated quantitatively in the future.

(3) Triggering mechanisms: The triggering process is the most important part of CME models, giving the observational fact that the characteristic time of the gradual free energy accumulation is much longer than that of its impulsive release. About 7 mechanisms have been proposed, and many of them are physically the same, e.g., the flux cancellation, the tether-cutting, and the breakout models (the breakout model is a kind of external tether cutting). Some triggering mechanisms involve resistive MHD processes (e.g., reconnection), and others involve only ideal MHD process. It is generally believed that magnetic free energy has been stored in the CME progenitors, and the triggering process, which can be ideal or resistive, does not supply much energy to the eruptions.

(4) Eruption: After the CME progenitor is triggered to rise, the core field moves up, which is characterized by the slowly ascending filament and/or overlying coronal loop. The ensuing evolution may bifurcate into two cases: (a) If a current sheet is formed below the core field and magnetic reconnection is excited in the current sheet, the evolution becomes eruptive, with flaring loops being formed below the reconnection site and the core field being ejected above the reconnection site. The ejection of the core field and the magnetic reconnection are experiencing a 
positive feedback interaction, leading to the impulsive phase of the flare and the strong acceleration of the core field. The fast ejection of the core field would stretch up the overlying magnetic field successively, forming the CME frontal loop above. As depicted by the standard CSHKP model, magnetic reconnection plays a crucial role in these, fast generally, events. In the case when the triggering of the CME is also due to reconnection, the whole process can be called two-step reconnection, as proposed for solar flares (Wang and Shi, 1993); (b) If magnetic reconnection is not sufficiently excited below the core field, the magnetic loop may stop or may have a chance to expand, either by ideal MHD instabilities (Chen, 1989; Török and Kliem, 2007; Rachmeler et al., 2009) or being slowly accelerated by the drag force of the ambient solar wind. In this case, the low corona would not show significant brightenings.

(5) Nature of the CME frontal loop: It was proposed that the CME frontal loop is a fast-mode shock wave front in the early time, which was later discarded, and replaced with the idea that they are erupting bright coronal loop or flux rope and the plasma pile-up immediately ahead. However, the very rare spectroscopic observations indicate that the CME frontal loop presents a mass velocity several times smaller than the apparent moving velocity, suggesting that the CME frontal loops might be plasma pile-up swept by waves, rather than being an erupting structure. A new idea was recently proposed based on the observational fact that CME frontal loops are cospatial with EIT waves. The new mechanism claims that the frontal loop of fast CMEs might neither be an expanding magnetic loop nor material swept by waves. It might be the density-enhanced pattern caused by the successive stretching of the field lines (see Section 4.4). If this is confirmed, the top part of the frontal loop in the early stage actually propagates outward with the local fast-mode wave speed, which is not a bulk velocity. Detailed modelings are strongly in need.

(6) Is magnetic reconnection necessary? Theoretically, no in the 3D situations. Practically, in many events, especially those that are interesting in the space weather context, reconnection is the crucial mechanism that enables the fast CME eruption and the associated solar flare. Note that there might be also some CME events in which magnetic reconnection plays a trivial role, compared to other effects like the solar wind and the intrinsic ideal MHD instability.

After finishing this review paper, I have one caution for the readers when scrutinizing the CME modelings in the past decades. When deriving the analytical solutions for CME eruptions, some details are missing, e.g., the reconnection outflow, and the frozen-in effect might be violated; When doing MHD numerical simulations, two factors are tricky and might significantly affect the conclusions: one is the numerical resistivity, and the other is the boundary conditions. The numerical resistivity makes it difficult to distinguish ideal MHD processes from resistive MHD processes, and the different treating of the boundary conditions, especially at the top boundary, may lead to completely different results in terms of whether an eruption can occur or not.

Finally, I have some comments for the future research:

(1) In order to understand and predict the CME initiation, the internal cause, e.g., the magnetic nonpotentiality, and the external cause, e.g., the emerging flux, should be considered together.

(2) Little attention was paid to the nature of the CME frontal loop, which is not fully understood yet. The improper fitting of a CME evolution to a flux rope model might be misleading in many cases in the literature. With the archived imaging and spectroscopic data and the soon-coming Solar Dynamics Observatory (SDO) observations with high spatio-temporal resolutions, we are close to disclosing the veil of the spectacular phenomenon, CMEs.

(3) It might be of great importance to predict how much energy and magnetic helicity can be released from a source region if it erupts as a CME.

Living Reviews in Solar Physics

http://www. livingreviews.org/lrsp-2011-1 


\section{Acknowledgements}

The author is grateful to the editors for encouraging me to write this review paper and to two anonymous referees for their detailed and constructive comments, which greatly improved the quality of the paper. The discussions with J. Chen, Y. Chen, T.G. Forbes, B.C. Low, S.T. Wu, and J. Zhang are beneficial. PFC has been financially supported by Chinese foundations 2011CB811402 and NSFC (11025314, 10878002, and 10933003) in the past years. SOHO is a project of international cooperation between ESA and NASA. We are also grateful to the BBSO and MLSO teams for making their data available. 


\section{References}

Alexander, D., Richardson, I.G. and Zurbuchen, T.H., 2006, "A Brief History of CME Science", Space Sci. Rev., 123, 3-11. [DOI], [ADS] (Cited on page 5.)

Aly, J.J., 1984, "On some properties of force-free magnetic fields in infinite regions of space", Astrophys. J., 283, 349-362. [DOI], [ADS] (Cited on page 10.)

Aly, J.J., 1990, "Quasi-static evolution of a force-free magnetic field", Comput. Phys. Commun., 59, 13-20. [DOI], [ADS] (Cited on page 25.)

Aly, J.J., 1991, "How much energy can be stored in a three-dimensional force-free magnetic field?", Astrophys. J. Lett., 375, L61-L64. [DOI], [ADS] (Cited on pages 10, 26, and 48.)

Amari, T. and Luciani, J.F., 1999, "Confined Disruption of a Three-dimensional Twisted Magnetic Flux Tube", Astrophys. J. Lett., 515, L81-L84. [DOI], [ADS] (Cited on page 16.)

Amari, T., Luciani, J.F., Aly, J.J. and Tagger, M., 1996, "Plasmoid formation in a single sheared arcade and application to coronal mass ejections", Astron. Astrophys., 306, 913. [ADS] (Cited on page 16.)

Amari, T., Luciani, J.F., Mikić, Z. and Linker, J., 2000, "A Twisted Flux Rope Model for Coronal Mass Ejections and Two-Ribbon Flares", Astrophys. J. Lett., 529, L49-L52. [DOI], [ADS] (Cited on pages 35 and 36.$)$

Amari, T., Luciani, J.F., Aly, J.J., Mikić, Z. and Linker, J., 2003a, "Coronal Mass Ejection: Initiation, Magnetic Helicity, and Flux Ropes. I. Boundary Motion-driven Evolution", Astrophys. J., 585, 1073-1086. [DOI], [ADS] (Cited on page 34.)

Amari, T., Luciani, J.F., Aly, J.J., Mikić, Z. and Linker, J., 2003b, "Coronal Mass Ejection: Initiation, Magnetic Helicity, and Flux Ropes. II. Turbulent Diffusion-driven Evolution", Astrophys. J., 595, 1231-1250. [DOI], [ADS] (Cited on pages 15 and 24.)

Amari, T., Luciani, J.F. and Aly, J.J., 2004, "Coronal Magnetohydrodynamic Evolution Driven by Subphotospheric Conditions", Astrophys. J. Lett., 615, L165-L168. [DOI], [ADS] (Cited on pages 31 and 40.)

Andrews, M.D., 2002, "The Front-to-Back Asymmetry of Coronal Emission", Solar Phys., 208, 317-324. [ADS] (Cited on page 62.)

Andrews, M.D. and Howard, R.A., 2001, "A two-Type Classification of Lasco Coronal Mass Ejection", Space Sci. Rev., 95, 147-163. [ADS] (Cited on page 51.)

Antiochos, S.K., Dahlburg, R.B. and Klimchuk, J.A., 1994, "The magnetic field of solar prominences", Astrophys. J. Lett., 420, L41-L44. [DOI], [ADS] (Cited on pages 16 and 17.)

Antiochos, S.K., DeVore, C.R. and Klimchuk, J.A., 1999, "A Model for Solar Coronal Mass Ejections", Astrophys. J., 510, 485-493. [DOI], [ADS], [arXiv:astro-ph/9807220] (Cited on pages 26 and 45.)

Anzer, U., 1978, "Can coronal loop transients be driven magnetically", Solar Phys., 57, 111-118. [DOI], [ADS] (Cited on page 39.)

Archontis, V. and Hood, A.W., 2008, "A Flux Emergence Model for Solar Eruptions", Astrophys. J. Lett., 674, L113-L116. [DOI], [ADS], [arXiv:0801.1649] (Cited on pages 29 and 31.) 
Archontis, V. and Török, T., 2008, "Eruption of magnetic flux ropes during flux emergence", Astron. Astrophys., 492, L35-L38. [DOI], [ADS], [arXiv:0811.1134] (Cited on page 26.)

Attrill, G., Nakwacki, M.S., Harra, L.K., van Driel-Gesztelyi, L., Mandrini, C.H., Dasso, S. and Wang, J., 2006, "Using the Evolution of Coronal Dimming Regions to Probe the Global Magnetic Field Topology", Solar Phys., 238, 117-139. [DOI], [ADS] (Cited on pages 11, 45, 48, and 54.)

Attrill, G.D.R., Harra, L.K., van Driel-Gesztelyi, L. and Démoulin, P., 2007, "Coronal 'Wave': Magnetic Footprint of a Coronal Mass Ejection?", Astrophys. J. Lett., 656, L101-L104. [DOI], [ADS] (Cited on pages 56 and 57.)

Attrill, G.D.R., van Driel-Gesztelyi, L., Démoulin, P., Zhukov, A.N., Steed, K., Harra, L.K., Mandrini, C.H. and Linker, J., 2008, "The Recovery of CME-Related Dimmings and the ICME's Enduring Magnetic Connection to the Sun", Solar Phys., 252, 349-372. [DOI], [ADS] (Cited on page 42.)

Aulanier, G. and Demoulin, P., 1998, "3-D magnetic configurations supporting prominences. I. The natural presence of lateral feet", Astron. Astrophys., 329, 1125-1137. [ADS] (Cited on pages 15 and 16.)

Aulanier, G., DeLuca, E.E., Antiochos, S.K., McMullen, R.A. and Golub, L., 2000, "The Topology and Evolution of the Bastille Day Flare", Astrophys. J., 540, 1126-1142. [DOI], [ADS] (Cited on page 27.)

Aulanier, G., DeVore, C.R. and Antiochos, S.K., 2002, "Prominence Magnetic Dips in ThreeDimensional Sheared Arcades", Astrophys. J. Lett., 567, L97-L101. [DOI], [ADS] (Cited on page 17.)

Aulanier, G., Török, T., Démoulin, P. and DeLuca, E.E., 2010, "Formation of Torus-Unstable Flux Ropes and Electric Currents in Erupting Sigmoids", Astrophys. J., 708, 314-333. [DOI], [ADS] (Cited on page 32.)

Ballai, I., 2007, "Global Coronal Seismology", Solar Phys., 246, 177-185. [DOI], [ADS], [arXiv:0704.1398] (Cited on page 54.)

Ballester, J.L., 2006, "Seismology of Prominence-Fine structures: Observations and Theory", Space Sci. Rev., 122, 129-135. [DOI], [ADS] (Cited on page 36.)

Bao, X.-M., Zhang, H.-Q. and Lin, J., 2006, "Formation of the CME Leading Edge Observed in the 2003 February 18 Event", Chin. J. Astron. Astrophys., 6, 741-750. [ADS] (Cited on page 60.)

Barnes, C.W. and Sturrock, P.A., 1972, "Force-Free Magnetic-Field Structures and Their Role in Solar Activity", Astrophys. J., 174, 659. [DOI], [ADS] (Cited on pages 25 and 48.)

Bateman, G., 1978, MHD Instabilities, MIT Press, Cambridge, MA (Cited on page 31.)

Baty, H., 2001, "On the MHD stability of the $m=1$ kink mode in solar coronal loops", Astron. Astrophys., 367, 321-325. [DOI], [ADS] (Cited on page 31.)

Berger, M.A., 1984, "Rigorous new limits on magnetic helicity dissipation in the solar corona", Geophys. Astrophys. Fluid Dyn., 30, 79-104. [DOI], [ADS] (Cited on pages 10 and 37.)

Biesecker, D.A., Myers, D.C., Thompson, B.J., Hammer, D.M. and Vourlidas, A., 2002, "Solar Phenomena Associated with 'EIT Waves"', Astrophys. J., 569, 1009-1015. [DOI], [ADS] (Cited on page 54.) 
Billings, D.E., 1966, A Guide to the Solar Corona, Academic Press, New York (Cited on page 6.)

Bommier, V. and Leroy, J.L., 1998, "Global Pattern of the Magnetic Field Vectors Above Neutral Lines from 1974 to 1982: Pic-du-Midi Observations of Prominences", in IAU Colloq. 167: New Perspectives on Solar Prominences, (Eds.) Webb, D.F., Schmieder, B., Rust, D.M., vol. 150 of ASP Conference Series, p. 434, Astronomical Society of the Pacific, San Francisco. [ADS] (Cited on page 13.)

Bruzek, A., 1951, "Beobachtungen über das Verhalten von Filamenten während chromosphärischer Eruptionen", Z. Astrophys., 28, 277-259. [ADS] (Cited on page 21.)

Cane, H.V., Kahler, S.W. and Sheeley Jr, N.R., 1986, "Interplanetary shocks preceded by solar filament eruptions", J. Geophys. Res., 91, 13,321-13,329. [DOI], [ADS] (Cited on page 51.)

Canfield, R.C., Hudson, H.S. and McKenzie, D.E., 1999, "Sigmoidal morphology and eruptive solar activity", Geophys. Res. Lett., 26, 627-630. [DOI], [ADS] (Cited on pages 15 and 18.)

Carmichael, H., 1964, "A Process for Flares", in The Physics of Solar Flares, Proceedings of the AAS-NASA Symposium held 28-30 October, 1963 at the Goddard Space Flight Center, Greenbelt, MD, (Ed.) Hess, W.N., vol. SP-50 of NASA Special Publicsations, p. 451, NASA Science and Technical Information Division, Washington, DC. [ADS] (Cited on page 12.)

Chen, A.-Q. and Zong, W.-G., 2009, "Relationship between CME velocities and X-ray fluxes of associated flares", Res. Astron. Astrophys., 9, 470-474. [DOI], [ADS] (Cited on page 53.)

Chen, A.Q., Yeh, C.T., Cheng, J.X. and Chen, P.F., 2005a, "A Reconsideration of the Classification of Two Types of CMEs", in Coronal and Stellar Mass Ejections, Proceedings of IAU Symposium 226, held in Beijing, China, September 13-17, 2004, (Eds.) Dere, K., Wang, J., Yan, Y., Proc. IAU, pp. 110-111, Cambridge University Press, Cambridge; New York. [DOI], [ADS], [Google Books] (Cited on page 51.)

Chen, A.Q., Chen, P.F. and Fang, C., 2006a, "On the CME velocity distribution", Astron. Astrophys., 456, 1153-1158. [DOI], [ADS] (Cited on pages 52 and 53.)

Chen, J., 1989, "Effects of toroidal forces in current loops embedded in a background plasma", Astrophys. J., 338, 453-470. [DOI], [ADS] (Cited on pages 29, 30, 31, 40, and 64.)

Chen, J., 1996, "Theory of prominence eruption and propagation: Interplanetary consequences", J. Geophys. Res., 101, 27,499-27,520. [DOI], [ADS] (Cited on pages 29, 30, 39, 58, and 61.)

Chen, J. and Krall, J., 2003, "Acceleration of coronal mass ejections", J. Geophys. Res., 108(A11), 1410. [DOI], [ADS] (Cited on page 51.)

Chen, J., Howard, R.A., Brueckner, G.E., Santoro, R., Krall, J., Paswaters, S.E., St Cyr, O.C., Schwenn, R., Lamy, P. and Simnett, G.M., 1997, "Evidence of an Erupting Magnetic Flux Rope: LASCO Coronal Mass Ejection of 1997 April 13", Astrophys. J. Lett., 490, L191. [DOI], [ADS] (Cited on page 15.)

Chen, J., Santoro, R.A., Krall, J., Howard, R.A., Duffin, R., Moses, J.D., Brueckner, G.E., Darnell, J.A. and Burkepile, J.T., 2000, "Magnetic Geometry and Dynamics of the Fast Coronal Mass Ejection of 1997 September 9", Astrophys. J., 533, 481-500. [DOI], [ADS] (Cited on pages 40 and 58.)

Chen, P.F., 2006, "The Relation between EIT Waves and Solar Flares", Astrophys. J. Lett., 641, L153-L156. [DOI], [ADS] (Cited on page 54.) 
Chen, P.F., 2008, "Initiation and propagation of coronal mass ejections", J. Astrophys. Astron., 29, 179-186. [DOI], [ADS], [arXiv:0712.3632] (Cited on pages 27, 53, and 54.)

Chen, P.F., 2009a, "The Relation Between EIT Waves and Coronal Mass Ejections", Astrophys. J. Lett., 698, L112-L115. [DOI], [ADS], [arXiv:0905.3272] (Cited on pages 6, 40, 41, 44, 54, 59, and 60.)

Chen, P.F., 2009b, "EIT waves and coronal magnetic field diagnostics", Sci. China Ser. G, 52 (11), 1785-1789. [DOI], [ADS], [arXiv:1001.0065] (Cited on page 54.)

Chen, P.F. and Fang, C., 2005, "EIT waves - A signature of global magnetic restructuring in CMEs", in Coronal and Stellar Mass Ejections, Proceedings of IAU Symposium 226, held in Beijing, China, September 13-17, 2004, (Eds.) Dere, K., Wang, J., Yan, Y., Proc. IAU, pp. 55-64, Cambridge University Press, Cambridge; New York. [DOI], [ADS], [Google Books] (Cited on page 41.)

Chen, P.F. and Shibata, K., 2000, "An Emerging Flux Trigger Mechanism for Coronal Mass Ejections", Astrophys. J., 545, 524-531. [DOI], [ADS] (Cited on pages 19, 27, 28, 35, 39, 42, and 52.)

Chen, P.F., Fang, C., Tang, Y.H. and Ding, M.D., 1999, "Simulation of Magnetic Reconnection with Heat Conduction", Astrophys. J., 513, 516-523. [DOI], [ADS] (Cited on page 46.)

Chen, P.F., Wu, S.T., Shibata, K. and Fang, C., 2002, "Evidence of EIT and Moreton Waves in Numerical Simulations", Astrophys. J. Lett., 572, L99-L102. [DOI], [ADS] (Cited on pages 41, $42,46,55,56,57$, and 59.)

Chen, P.F., Ding, M.D. and Fang, C., 2005b, "Synthesis of CME-Associated Moreton and EIT Wave Features from MHD Simulations", Space Sci. Rev., 121, 201-211. [DOI], [ADS] (Cited on page 55.)

Chen, P.F., Fang, C. and Shibata, K., 2005c, "A Full View of EIT Waves", Astrophys. J., 622, 1202-1210. [DOI], [ADS] (Cited on pages 41, 42, 46, 55, and 59.)

Chen, P.F., Fang, C. and Shibata, K., 2006b, "Why are there stationary EIT wave fronts", Adv. Space Res., 38, 456-460. [DOI], [ADS] (Cited on page 55.)

Chen, P.F., Innes, D.E. and Solanki, S.K., 2008, "SOHO/SUMER observations of prominence oscillation before eruption", Astron. Astrophys., 484, 487-493. [DOI], [ADS], [arXiv:0802.1961] (Cited on pages 22 and 29.)

Cheng, J.-X., Fang, C., Chen, P.F. and Ding, M.-D., 2005, "Two Sympathetic Homologous CMEs on 2002 May 22", Chin. J. Astron. Astrophys., 5(3), 265-272. [DOI], [ADS] (Cited on page 36.)

Chertok, I.M., Kahler, S., Aurass, H. and Gnezdilov, A.A., 2001, "Sharp Decreases of Solar Metric Radio Storm Emission", Solar Phys., 202, 337-354. [ADS] (Cited on page 20.)

Ciaravella, A., Raymond, J.C., Li, J., Reiser, P., Gardner, L.D., Ko, Y.-K. and Fineschi, S., 2002, "Elemental Abundances and Post-Coronal Mass Ejection Current Sheet in a Very Hot Active Region", Astrophys. J., 575, 1116-1130. [DOI], [ADS] (Cited on page 50.)

Ciaravella, A., Raymond, J.C. and Kahler, S.W., 2006, "Ultraviolet Properties of Halo Coronal Mass Ejections: Doppler Shifts, Angles, Shocks, and Bulk Morphology", Astrophys. J., 652, 774-792. [DOI], [ADS] (Cited on pages 45 and 59.) 
Cliver, E.W., 1995, "Solar activity and geomagnetic storms: From M regions and flares to coronal holes and CMEs", Eos Trans. AGU, 76(8), 75-75. [DOI], [ADS] (Cited on page 5.)

Cliver, E.W. and Hudson, H.S., 2002, "CMEs: How do the puzzle pieces fit together?", J. Atmos. Sol.-Terr. Phys., 64, 231-252. [DOI], [ADS] (Cited on page 51.)

Cliver, E.W., Kahler, S.W. and McIntosh, P.S., 1983, "Solar proton flares with weak impulsive phases", Astrophys. J., 264, 699-707. [DOI], [ADS] (Cited on page 50.)

Cliver, E.W., Webb, D.F. and Howard, R.A., 1999, "On the origin of solar metric type II bursts", Solar Phys., 187, 89-114. [DOI], [ADS] (Cited on page 46.)

Cliver, E.W., Laurenza, M., Storini, M. and Thompson, B.J., 2005, "On the Origins of Solar EIT Waves", Astrophys. J., 631, 604-611. [DOI], [ADS] (Cited on page 54.)

Cohen, O., Attrill, G.D.R., Manchester IV, W.B. and Wills-Davey, M.J., 2009, "Numerical Simulation of an EUV Coronal Wave Based on the 2009 February 13 CME Event Observed by STEREO", Astrophys. J., 705, 587-602. [DOI], [ADS], [arXiv:0909.3095] (Cited on pages 45 and 57.)

Collier Cameron, A. and Robinson, R.D., 1989, "Fast $\mathrm{H} \alpha$ variations on a rapidly rotating, cool main-sequence star - II. Cloud formation and ejection", Mon. Not. R. Astron. Soc., 238, 657674. [ADS] (Cited on page 5.)

Cremades, H. and Bothmer, V., 2004, "On the three-dimensional configuration of coronal mass ejections", Astron. Astrophys., 422, 307-322. [DOI], [ADS] (Cited on page 11.)

Crosby, N., Vilmer, N., Lund, N., Klein, K.-L. and Sunyaev, R., 1996, "Deka-KeV X-Ray Emission Associated with the Onset of Radio Noise Storms", Solar Phys., 167, 333-348. [DOI], [ADS] (Cited on page 19.)

Dai, Y., Auchère, F., Vial, J.-C., Tang, Y.H. and Zong, W.G., 2010, "Large-scale ExtremeUltraviolet Disturbances Associated with a Limb Coronal Mass Ejection", Astrophys. J., 708, 913-919. [DOI], [ADS] (Cited on page 59.)

Delannée, C., 2000, "Another View of the EIT Wave Phenomenon", Astrophys. J., 545, 512-523. [DOI], [ADS] (Cited on page 54.)

Delannée, C., 2009, "The role of small versus large scale magnetic topologies in global waves", Astron. Astrophys., 495, 571-575. [DOI], [ADS] (Cited on pages 54 and 56.)

Delannée, C. and Aulanier, G., 1999, "CME Associated with Transequatorial Loops and a Bald Patch Flare", Solar Phys., 190, 107-129. [DOI], [ADS] (Cited on pages 41, 45, 54, and 57.)

Delannée, C., Hochedez, J.-F. and Aulanier, G., 2007, "Stationary parts of an EIT and Moreton wave: a topological model", Astron. Astrophys., 465, 603-612. [DOI], [ADS] (Cited on pages 45 and 54.)

Delannée, C., Török, T., Aulanier, G. and Hochedez, J.-F., 2008, "A New Model for Propagating Parts of EIT Waves: A Current Shell in a CME", Solar Phys., 247, 123-150. [DOI], [ADS] (Cited on page 57. .

Démoulin, P., 2007, "Recent theoretical and observational developments in magnetic helicity studies", Adv. Space Res., 39, 1674-1693. [DOI], [ADS] (Cited on page 38.) 
Démoulin, P. and Aulanier, G., 2010, "Criteria for Flux Rope Eruption: Non-equilibrium Versus Torus Instability", Astrophys. J., 718, 1388-1399. [DOI], [ADS], [arXiv:1006.1785 [astro-ph.SR]] (Cited on page 34.)

Démoulin, P., Mandrini, C.H., van Driel-Gesztelyi, L., Thompson, B.J., Plunkett, S., Kovári, Z., Aulanier, G. and Young, A., 2002, "What is the source of the magnetic helicity shed by CMEs? The long-term helicity budget of AR 7978", Astron. Astrophys., 382, 650-665. [DOI], [ADS] (Cited on page 22.)

Deng, Y., Wang, J., Yan, Y. and Zhang, J., 2001, "Evolution of Magnetic Nonpotentiality in NOAA AR 9077", Solar Phys., 204, 11-26. [DOI], [ADS] (Cited on page 25.)

Dere, K., Brueckner, G., Howard, R., Koomen, M., Korendyke, C., Kreplin, R., Michels, D., Moses, J., Moulton, N., Socker, D., St Cyr, O., Delaboudinière, J., Artzner, G., Brunaud, J., Gabriel, A., Hochedez, J., Millier, F., Song, X., Chauvineau, J., Marioge, J., Defise, J., Jamar, C., Rochus, P., Catura, R., Lemen, J., Gurman, J., Neupert, W., Clette, F., Cugnon, P., Van Dessel, E., Lamy, P., Llebaria, A., Schwenn, R. and Simnett, G., 1997, "EIT and LASCO Observations of the Initiation of a Coronal Mass Ejection", Solar Phys., 175, 601-612. [DOI], [ADS] (Cited on page 6.)

Dere, K.P., Brueckner, G.E., Howard, R.A., Michels, D.J. and Delaboudiniere, J.P., 1999, "LASCO and EIT Observations of Helical Structure in Coronal Mass Ejections", Astrophys. J., 516, 465474. [DOI], [ADS] (Cited on page 15.)

DeRosa, M.L., Schrijver, C.J., Barnes, G., Leka, K.D., Lites, B.W., Aschwanden, M.J., Amari, T., Canou, A., McTiernan, J.M., Régnier, S., Thalmann, J.K., Valori, G., Wheatland, M.S., Wiegelmann, T., Cheung, M.C.M., Conlon, P.A., Fuhrmann, M., Inhester, B. and Tadesse, T., 2009, "A Critical Assessment of Nonlinear Force-Free Field Modeling of the Solar Corona for Active Region 10953", Astrophys. J., 696, 1780-1791. [DOI], [ADS], [arXiv:0902.1007] (Cited on page 11.)

Ding, J.Y., Hu, Y.Q. and Wang, J.X., 2006, "Catastrophic Behavior of Multiple Coronal Flux Rope System", Solar Phys., 235, 223-234. [DOI], [ADS] (Cited on page 36.)

Downs, C., Roussev, I.I., van der Holst, B., Lugaz, N., Sokolov, I.V. and Gombosi, T.I., 2011, "Studying Extreme Ultraviolet Wave Transients with a Digital Laboratory: Direct Comparison of Extreme Ultraviolet Wave Observations to Global Magnetohydrodynamic Simulations", Astrophys. J., 728, 2. [DOI], [ADS] (Cited on page 55.)

Dubey, G., van der Holst, B. and Poedts, S., 2006, "The initiation of coronal mass ejections by magnetic flux emergence", Astron. Astrophys., 459, 927-934. [DOI], [ADS] (Cited on page 29.)

Dungey, J.W., 1953, "Conditions for the occurrence of electrical discharges in astrophysical systems", Philos. Mag., 44(354), 725-738 (Cited on page 34.)

Emslie, A.G., Kucharek, H., Dennis, B.R., Gopalswamy, N., Holman, G.D., Share, G.H., Vourlidas, A., Forbes, T.G., Gallagher, P.T., Mason, G.M., Metcalf, T.R., Mewaldt, R.A., Murphy, R.J., Schwartz, R.A. and Zurbuchen, T.H., 2004, "Energy partition in two solar flare/CME events", J. Geophys. Res., 109, A10104. [DOI], [ADS] (Cited on page 8.)

Engvold, O., 1989, "Prominence environment", in Dynamics and Structure of Quiescent Solar Prominences, Proceedings of the Workshop, Palma de Mallorca, Spain, November 1987, (Ed.) Priest, E.R., vol. 150 of Astrophysics and Space Science Library, pp. 47-76, Kluwer Academic, Dordrecht. [ADS], [Google Books] (Cited on page 42.) 
Eselevich, M.V. and Eselevich, V.G., 2008, "On formation of a shock wave in front of a coronal mass ejection with velocity exceeding the critical one", Geophys. Res. Lett., 35, 22105. [DOI], [ADS] (Cited on page 45.)

Eto, S., Isobe, H., Narukage, N., Asai, A., Morimoto, T., Thompson, B., Yashiro, S., Wang, T., Kitai, R., Kurokawa, H. and Shibata, K., 2002, "Relation between a Moreton Wave and an EIT Wave Observed on 1997 November 4", Publ. Astron. Soc. Japan, 54, 481-491. [ADS] (Cited on pages 36 and 54.)

Falconer, D.A., Moore, R.L. and Gary, G.A., 2002, "Correlation of the Coronal Mass Ejection Productivity of Solar Active Regions with Measures of Their Global Nonpotentiality from Vector Magnetograms: Baseline Results", Astrophys. J., 569, 1016-1025. [DOI], [ADS] (Cited on page 37.)

Fan, Y., 2005, "Coronal Mass Ejections as Loss of Confinement of Kinked Magnetic Flux Ropes", Astrophys. J., 630, 543-551. [DOI], [ADS] (Cited on page 49.)

Fan, Y. and Gibson, S.E., 2007, "Onset of Coronal Mass Ejections Due to Loss of Confinement of Coronal Flux Ropes", Astrophys. J., 668, 1232-1245. [DOI], [ADS] (Cited on pages 31, 32, and 40.)

Fan, Y. and Low, B.C., 2003, "Dynamics of CME Driven by a Buoyant Prominence Flux Tube", in Current Theoretical Models and Future High Resolution Solar Observations: Preparing for ATST, 11-15 March 2002 at NSO, Sunspot, New Mexico, USA, (Eds.) Pevtsov, A.A., Uitenbroek, H., vol. 286 of ASP Conference Series, p. 347, Astronomical Society of the Pacific, San Francisco. [ADS] (Cited on page 36.)

Feynman, J. and Martin, S.F., 1995, "The initiation of coronal mass ejections by newly emerging magnetic flux", J. Geophys. Res., 100, 3355-3367. [DOI], [ADS] (Cited on pages 18, 19, and 27.)

Feynman, J. and Ruzmaikin, A., 2004, "A High-Speed Erupting-Prominence CME: A Bridge Between Types", Solar Phys., 219, 301-313. [DOI], [ADS] (Cited on page 52.)

Filippov, B. and Koutchmy, S., 2008, "Causal relationships between eruptive prominences and coronal mass ejections", Ann. Geophys., 26, 3025-3031. [ADS], [arXiv:0711.4752] (Cited on page 18.)

Fisher, R.R. and Munro, R.H., 1984, "Coronal transient geometry. I. The flare-associated event of 1981 March 25", Astrophys. J., 280, 428-439. [DOI], [ADS] (Cited on pages 44 and 58.)

Forbes, T.G., 2000, "A review on the genesis of coronal mass ejections", J. Geophys. Res., 105, 23,153-23,166. [DOI], [ADS] (Cited on pages 5, 9, 10, 11, 12, 23, 30, 44, 48, and 59.)

Forbes, T.G. and Isenberg, P.A., 1991, "A catastrophe mechanism for coronal mass ejections", Astrophys. J., 373, 294-307. [DOI], [ADS] (Cited on pages 32 and 33.)

Forbes, T.G. and Priest, E.R., 1995, "Photospheric Magnetic Field Evolution and Eruptive Flares", Astrophys. J., 446, 377. [DOI], [ADS] (Cited on pages 34 and 35.)

Forbes, T.G., Priest, E.R. and Isenberg, P.A., 1994, "On the maximum energy release in flux-rope models of eruptive flares", Solar Phys., 150, 245-266. [DOI], [ADS] (Cited on page 32.)

Forbes, T.G., Linker, J.A., Chen, J., Cid, C., Kóta, J., Lee, M.A., Mann, G., Mikić, Z., Potgieter, M.S., Schmidt, J.M., Siscoe, G.L., Vainio, R., Antiochos, S.K. and Riley, P., 2006, "CME Theory and Models", Space Sci. Rev., 123, 251-302. [DOI], [ADS] (Cited on pages 5, 36, and 44.)

Living Reviews in Solar Physics

http://www. livingreviews.org/lrsp-2011-1 
Gallagher, P.T. and Long, D.M., 2010, "Large-scale Bright Fronts in the Solar Corona: A Review of 'EIT waves"', Space Sci. Rev.. [DOI], [ADS], [arXiv:1006.0140] (Cited on pages 53 and 54.)

Gary, G.A., 2001, "Plasma Beta above a Solar Active Region: Rethinking the Paradigm", Solar Phys., 203, 71-86. [ADS] (Cited on pages 10 and 50.)

Gibson, S.E. and Fan, Y., 2006, "The Partial Expulsion of a Magnetic Flux Rope", Astrophys. J. Lett., 637, L65-L68. [DOI], [ADS] (Cited on page 18.)

Gibson, S.E. and Fan, Y., 2008, "Partially ejected flux ropes: Implications for interplanetary coronal mass ejections", J. Geophys. Res., 113, 9103. [DOI], [ADS] (Cited on pages 11 and 45.)

Gibson, S.E. and Low, B.C., 1998, "A Time-Dependent Three-Dimensional Magnetohydrodynamic Model of the Coronal Mass Ejection", Astrophys. J., 493, 460. [DOI], [ADS] (Cited on page 48.)

Gibson, S.E., Fan, Y., Török, T. and Kliem, B., 2006a, "The Evolving Sigmoid: Evidence for Magnetic Flux Ropes in the Corona Before, During, and After CMEs", Space Sci. Rev., 124, 131-144. [DOI], [ADS] (Cited on page 15.)

Gibson, S.E., Foster, D., Burkepile, J., de Toma, G. and Stanger, A., 2006b, "The Calm before the Storm: The Link between Quiescent Cavities and Coronal Mass Ejections", Astrophys. J., 641, 590-605. [DOI], [ADS] (Cited on pages 14, 41, and 58.)

Gilbert, H.R., Holzer, T.E., Burkepile, J.T. and Hundhausen, A.J., 2000, "Active and Eruptive Prominences and Their Relationship to Coronal Mass Ejections", Astrophys. J., 537, 503-515. [DOI], [ADS] (Cited on pages 18 and 58.)

Glover, A., Harra, L.K., Matthews, S.A., Hori, K. and Culhane, J.L., 2001, "Long term evolution of a non-active region sigmoid and its CME activity", Astron. Astrophys., 378, 239-246. [DOI], [ADS] (Cited on page 10.)

Glover, A., Ranns, N.D.R., Brown, D.S., Harra, L.K., Matthews, S.A. and Culhane, J.L., 2002, "The magnetic topology of a sigmoid", J. Atmos. Sol.-Terr. Phys., 64, 497-504. [DOI], [ADS] (Cited on page 15.)

Gold, T. and Hoyle, F., 1960, "On the origin of solar flares", Mon. Not. R. Astron. Soc., 120, 89. [ADS] (Cited on page 30.)

Gopalswamy, N. and Kundu, M.R., 1992, "Estimation of the mass of a coronal mass ejection from radio observations", Astrophys. J. Lett., 390, L37-L39. [DOI], [ADS] (Cited on page 6.)

Gopalswamy, N., Kundu, M.R., Hanaoka, Y., Enome, S., Lemen, J.R. and Akioka, M., 1996, "Yohkoh/SXT observations of a coronal mass ejection near the solar surface", New Astronomy, 1, 207-213. [DOI], [ADS] (Cited on page 6.)

Gopalswamy, N., Lara, A., Lepping, R.P., Kaiser, M.L., Berdichevsky, D. and St Cyr, O.C., 2000, "Interplanetary acceleration of coronal mass ejections", Geophys. Res. Lett., 27, 145-148. [DOI], [ADS] (Cited on page 43.)

Gopalswamy, N., Yashiro, S., Kaiser, M.L., Howard, R.A. and Bougeret, J.-L., 2001, "Characteristics of coronal mass ejections associated with long-wavelength type II radio bursts", J. Geophys. Res., 106, 29,219-29,230. [DOI], [ADS] (Cited on page 45.) 
Gopalswamy, N., Lara, A., Yashiro, S., Nunes, S. and Howard, R.A., 2003, "Coronal mass ejection activity during solar cycle 23", in Solar Variability as an Input to the Earth's Environment, International Solar Cycle Studies (ISCS) Symposium, 23-28 June 2003, Tatranská Lomnica, Slovak Republic, (Ed.) Wilson, A., vol. SP-535 of ESA Special Publication, pp. 403-414, ESA Publications Division, Noordwijk. [ADS] (Cited on pages 7 and 9.)

Gopalswamy, N., Mikić, Z., Maia, D., Alexander, D., Cremades, H., Kaufmann, P., Tripathi, D. and Wang, Y.-M., 2006, "The Pre-CME Sun", Space Sci. Rev., 123, 303-339. [DOI], [ADS] (Cited on page 18.)

Gosling, J.T., 1993, "The solar flare myth", J. Geophys. Res., 98, 18,937-18,950. [DOI], [ADS] (Cited on page 5.)

Gosling, J.T., 1999, "The Role of Reconnection in the Formation of Flux Ropes in the Solar Wind", in Magnetic Helicity in Space and Laboratory Plasmas, (Eds.) Brown, M.R., Canfield, R.C., Pevtsov, A.A., vol. 111 of Geophysical Monograph, pp. 205-212, American Geophysical Union, Washington, DC. [ADS], [Google Books] (Cited on page 15.)

Gosling, J.T., Hildner, E., MacQueen, R.M., Munro, R.H., Poland, A.I. and Ross, C.L., 1976, "The speeds of coronal mass ejection events", Solar Phys., 48, 389-397. [DOI], [ADS] (Cited on pages 50 and 51.)

Gosling, J.T., Baker, D.N., Bame, S.J., Feldman, W.C., Zwickl, R.D. and Smith, E.J., 1987, "Bidirectional solar wind electron heat flux events", J. Geophys. Res., 92, 8519-8535. [DOI], [ADS] (Cited on page 48.)

Grechnev, V.V., Uralov, A.M., Slemzin, V.A., Chertok, I.M., Kuzmenko, I.V. and Shibasaki, K., 2008, "Absorption Phenomena and a Probable Blast Wave in the 13 July 2004 Eruptive Event", Solar Phys., 253, 263-290. [DOI], [ADS], [arXiv:0811.0899] (Cited on page 54.)

Guo, Y., Schmieder, B., Démoulin, P., Wiegelmann, T., Aulanier, G., Török, T. and Bommier, V., 2010, "Coexisting Flux Rope and Dipped Arcade Sections Along One Solar Filament", Astrophys. J., 714, 343-354. [DOI], [ADS] (Cited on page 15.)

Harra, L.K. and Sterling, A.C., 2001, "Material Outflows from Coronal Intensity 'Dimming Regions' during Coronal Mass Ejection Onset", Astrophys. J. Lett., 561, L215-L218. [DOI], [ADS] (Cited on pages 8, 40, and 42.)

Harra, L.K. and Sterling, A.C., 2003, "Imaging and Spectroscopic Investigations of a Solar Coronal Wave: Properties of the Wave Front and Associated Erupting Material", Astrophys. J., 587, 429-438. [DOI], [ADS] (Cited on page 55.)

Harrison, R.A., 1986, "Solar coronal mass ejections and flares", Astron. Astrophys., 162, 283-291. [ADS] (Cited on page 44.)

Harrison, R.A., 1995, "The nature of solar flares associated with coronal mass ejection", Astron. Astrophys., 304, 585. [ADS] (Cited on pages 8, 9, and 12.)

Harrison, R.A. and Lyons, M., 2000, "A spectroscopic study of coronal dimming associated with a coronal mass ejection", Astron. Astrophys., 358, 1097-1108. [ADS] (Cited on pages 11 and 40.)

Harrison, R.A., Davis, C.J. and Davies, J.A., 2009, "Pre-CME Onset Fuses - Do the STEREO Heliospheric Imagers Hold the Clues to the CME Onset Process?", Solar Phys., 259, 277-296. [DOI], [ADS] (Cited on page 22.) 
He, H. and Wang, H., 2008, "Nonlinear force-free coronal magnetic field extrapolation scheme based on the direct boundary integral formulation", J. Geophys. Res., 113, A05S90. [DOI], [ADS], [arXiv:0704.0156] (Cited on page 15.)

Heyvaerts, J., Priest, E.R. and Rust, D.M., 1977, "An emerging flux model for the solar flare phenomenon", Astrophys. J., 216, 123-137. [DOI], [ADS] (Cited on page 29.)

Hiei, E., Hundhausen, A.J. and Sime, D.G., 1993, "Reformation of a coronal helmet streamer by magnetic reconnection after a coronal mass ejection", Geophys. Res. Lett., 20, 2785-2788. [DOI], [ADS] (Cited on pages 9 and 52.)

Hirayama, T., 1974, "Theoretical Model of Flares and Prominences. I: Evaporating Flare Model", Solar Phys., 34, 323-338. [DOI], [ADS] (Cited on page 12.)

Hood, A.W. and Priest, E.R., 1979, "Kink instability of solar coronal loops as the cause of solar flares", Solar Phys., 64, 303-321. [DOI], [ADS] (Cited on page 30.)

Hood, A.W. and Priest, E.R., 1980, "Magnetic instability of coronal arcades as the origin of tworibbon flares", Solar Phys., 66, 113-134. [DOI], [ADS] (Cited on page 13.)

House, L.L., Wagner, W.J., Hildner, E., Sawyer, C. and Schmidt, H.U., 1981, "Studies of the corona with the Solar Maximum Mission coronagraph/polarimeter", Astrophys. J. Lett., 244, L117-L121. [DOI], [ADS] (Cited on pages 6 and 58.)

Howard, R.A., Michels, D.J., Sheeley Jr, N.R. and Koomen, M.J., 1982, "The observation of a coronal transient directed at earth", Astrophys. J. Lett., 263, L101-L104. [DOI], [ADS] (Cited on pages 44,58 , and 61 .)

$\mathrm{Hu}$, Y.Q., 2001, "Catastrophe of coronal magnetic flux ropes in partially open magnetic fields", Solar Phys., 200, 115-126. [DOI], [ADS] (Cited on page 34.)

Hu, Y.Q., Li, G.Q. and Xing, X.Y., 2003, "Equilibrium and catastrophe of coronal flux ropes in axisymmetrical magnetic field", J. Geophys. Res., 108, 1072. [DOI], [ADS] (Cited on page 48.)

Hudson, H.S. and Cliver, E.W., 2001, "Observing coronal mass ejections without coronagraphs", J. Geophys. Res., 106, 25,199-25,214. [DOI], [ADS] (Cited on pages 6 and 12.)

Hudson, H.S., Acton, L.W. and Freeland, S.L., 1996, "A Long-Duration Solar Flare with Mass Ejection and Global Consequences", Astrophys. J., 470, 629. [DOI], [ADS] (Cited on page 6.)

Hudson, H.S., Acton, L.W., Harvey, K.L. and McKenzie, D.E., 1999, "A Stable Filament Cavity with a Hot Core", Astrophys. J. Lett., 513, L83-L86. [DOI], [ADS] (Cited on pages 15 and 41.)

Hundhausen, A.J., 1993, "Sizes and Locations of Coronal Mass Ejections: SMM Observations From 1980 and 1984-1989", J. Geophys. Res., 98(A8), 13,177-13,200. [DOI], [ADS] (Cited on pages 6 and 18.)

Hundhausen, A.J., 1997, "Coronal Mass Ejections", in Cosmic Winds and the Heliosphere, (Eds.) Jokipii, J.R., Sonett, C.P., Giampapa, M.S., Space Science Series, pp. 259-296, Arizona University Press, Tucson (Cited on page 53.)

Hundhausen, A.J., Sawyer, C.B., House, L., Illing, R.M.E. and Wagner, W.J., 1984, "Coronal Mass Ejections Observed During the Solar Maximum Mission: Latitude Distribution and Rate of Occurrence", J. Geophys. Res., 89, 2639-2646. [DOI], [ADS] (Cited on pages 6 and 58.) 
Hundhausen, A.J., Burkepile, J.T. and St Cyr, O.C., 1994, "Speeds of coronal mass ejections: SMM observations from 1980 and 1984-1989", J. Geophys. Res., 99, 6543-6552. [DOI], [ADS] (Cited on page 50.)

Illing, R.M.E. and Hundhausen, A.J., 1983, "Possible observation of a disconnected magnetic structure in a coronal transient", J. Geophys. Res., 88, 10,210-10,214. [DOI], [ADS] (Cited on page 15.)

Illing, R.M.E. and Hundhausen, A.J., 1985, "Observation of a coronal transient from 1.2 to 6 solar radii", J. Geophys. Res., 90, 275-282. [DOI], [ADS] (Cited on pages 6 and 40.)

Illing, R.M.E. and Hundhausen, A.J., 1986, "Disruption of a coronal streamer by an eruptive prominence and coronal mass ejection", J. Geophys. Res., 91, 10,951-10,960. [DOI], [ADS] (Cited on page 41.)

Inhester, B., Birn, J. and Hesse, M., 1992, "The evolution of line-tied coronal arcades including a converging footpoint motion", Solar Phys., 138, 257-281. [DOI], [ADS] (Cited on page 34.)

Inoue, S. and Kusano, K., 2006, "Three-dimensional Simulation Study of Flux Rope Dynamics in the Solar Corona", Astrophys. J., 645, 742-756. [DOI], [ADS] (Cited on pages 30, 31, and 49.)

Isenberg, P.A., Forbes, T.G. and Demoulin, P., 1993, "Catastrophic Evolution of a Force-free Flux Rope: A Model for Eruptive Flares", Astrophys. J., 417, 368. [DOI], [ADS] (Cited on page 34.)

Jackson, B.V., 1985, "Imaging of coronal mass ejections by the HELIOS spacecraft", Solar Phys., 100, 563-574. [DOI], [ADS] (Cited on page 6.)

Jackson, B.V. and Hildner, E., 1978, "Forerunners: Outer rims of solar coronal transients", Solar Phys., 60, 155-170. [DOI], [ADS] (Cited on page 45.)

Jackson, B.V., Sheridan, K.V., Dulk, G.A. and McLean, D.J., 1978, "A possible association of solar type III bursts and white light transients", Proc. Astron. Soc. Australia, 3, 241. [ADS] (Cited on pages 20 and 21.)

Jacobs, C., Poedts, S. and van der Holst, B., 2006, "The effect of the solar wind on CME triggering by magnetic foot point shearing", Astron. Astrophys., 450, 793-803. [DOI], [ADS] (Cited on page 25.)

Ji, H., Wang, H., Schmahl, E.J., Moon, Y.-J. and Jiang, Y., 2003, "Observations of the Failed Eruption of a Filament", Astrophys. J. Lett., 595, L135-L138. [DOI], [ADS] (Cited on page 31.)

Jiang, Y., Ji, H., Wang, H. and Chen, H., 2003, "H $\alpha$ Dimmings Associated with the X1.6 Flare and Halo Coronal Mass Ejection on 2001 October 19", Astrophys. J. Lett., 597, L161-L164. [DOI], [ADS] (Cited on page 42.)

Jin, M., Ding, M.D., Chen, P.F., Fang, C. and Imada, S., 2009, "Coronal Mass Ejection Induced Outflows Observed with Hinode/EIS", Astrophys. J., 702, 27-38. [DOI], [ADS] (Cited on page 42.)

Jing, J., Yurchyshyn, V.B., Yang, G., Xu, Y. and Wang, H., 2004, "On the Relation between Filament Eruptions, Flares, and Coronal Mass Ejections", Astrophys. J., 614, 1054-1062. [DOI], [ADS] (Cited on pages 9 and 19.)

Jing, J., Chen, P.F., Wiegelmann, T., Xu, Y., Park, S.-H. and Wang, H., 2009, "Temporal Evolution of Free Magnetic Energy Associated with Four X-Class Flares", Astrophys. J., 696, 84-90. [DOI], [ADS] (Cited on pages 10 and 37.) 
Joshi, V. and Srivastava, N., 2007, "On the study of kinematics of eruptive quiescent prominences observed in He $304 \AA "$ ", Bull. Astron. Soc. India, 35, 447-455. [ADS] (Cited on page 39.)

Kahler, S.W., Cliver, E.W., Cane, H.V., McGuire, R.E., Stone, R.G. and Sheeley Jr, N.R., 1986, "Solar filament eruptions and energetic particle events", Astrophys. J., 302, 504-510. [DOI], [ADS] (Cited on page 50.)

Kahler, S.W., Moore, R.L., Kane, S.R. and Zirin, H., 1988, "Filament eruptions and the impulsive phase of solar flares", Astrophys. J., 328, 824-829. [DOI], [ADS] (Cited on pages 38, 40, and 52.)

Karlický, M., Fárník, F. and Krucker, S., 2004, "High-frequency slowly drifting structures and X-ray sources observed by RHESSI", Astron. Astrophys., 419, 365-373. [DOI], [ADS] (Cited on page 46.)

Khan, J.I. and Hudson, H.S., 2000, "Homologous sudden disappearances of transequatorial interconnecting loops in the solar corona", Geophys. Res. Lett., 27, 1083. [DOI], [ADS] (Cited on page 13.)

Kienreich, I.W., Temmer, M. and Veronig, A.M., 2009, "STEREO Quadrature Observations of the Three-Dimensional Structure and Driver of a Global Coronal Wave", Astrophys. J. Lett., 703, L118-L122. [DOI], [ADS], [arXiv:0908.3571] (Cited on page 54.)

Kippenhahn, R. and Schlüter, A., 1957, "Eine Theorie der solaren Filamente", Z. Astrophys., 43, 36-62. [ADS] (Cited on page 17.)

Klassen, A., Aurass, H., Mann, G. and Thompson, B.J., 2000, "Catalogue of the 1997 SOHO-EIT coronal transient waves and associated type II radio burst spectra", Astron. Astrophys. Suppl., 141, 357-369. [DOI], [ADS] (Cited on pages 53 and 54.)

Klassen, A., Bothmer, V., Mann, G., Reiner, M.J., Krucker, S., Vourlidas, A. and Kunow, H., 2002, "Solar energetic electron events and coronal shocks", Astron. Astrophys., 385, 1078-1088. [DOI], [ADS] (Cited on page 21.)

Klein, K.-L., Aurass, H., Soru-Escaut, I. and Kalman, B., 1997, "Electron acceleration sites in a large-scale coronal structure", Astron. Astrophys., 320, 612-619. [ADS] (Cited on page 21.)

Kliem, B. and Török, T., 2006, "Torus Instability", Phys. Rev. Lett., 96, 255002. [DOI], [ADS], [arXiv:physics/0605217] (Cited on pages 30,31, and 40.)

Klimchuk, J.A., 1990, "Shear-induced inflation of coronal magnetic fields", Astrophys. J., 354, 745-754. [DOI], [ADS] (Cited on page 25.)

Klimchuk, J.A., 2001, "Theory of Coronal Mass Ejections", in Space Weather, (Eds.) Song, P., Singer, H.J., Siscoe, G.L., vol. 125 of Geophysical Monograph, p. 143, American Geophysical Union, Washington, DC. [ADS] (Cited on page 5.)

Klimchuk, J.A. and Sturrock, P.A., 1989, "Force-free magnetic fields: Is there a 'loss of equilibrium'?", Astrophys. J., 345, 1034-1041. [DOI], [ADS] (Cited on page 35.)

Klimchuk, J.A., Acton, L.W., Harvey, K.L., Hudson, H.S., Kluge, K.L., Sime, D.G., Strong, K.T. and Watanabe, T., 1994, "Coronal Eruptions Observed by YOHKOH", in X-Ray Solar Physics from Yohkoh, Proceedings of the International Symposium on the Yohkoh Scientific Results, on February 23-25, 1993, Sagamihara, Kanagawa, (Eds.) Uchida, Y., Watanabe, T., Shibata, K., Hudson, H.S., vol. 10 of Frontiers Science Series, p. 181, Universal Academy Press, Tokyo. [ADS] (Cited on page 6.) 
Kopp, R.A. and Pneuman, G.W., 1976, "Magnetic reconnection in the corona and the loop prominence phenomenon", Solar Phys., 50, 85-98. [DOI], [ADS] (Cited on page 12.)

Krall, J. and Chen, J., 2005, "Density Structure of a Preeruption Coronal Flux Rope", Astrophys. J., 628, 1046-1055. [DOI], [ADS] (Cited on page 58.)

Krall, J., Chen, J., Duffin, R.T., Howard, R.A. and Thompson, B.J., 2001, "Erupting Solar Magnetic Flux Ropes: Theory and Observation", Astrophys. J., 562, 1045-1057. [DOI], [ADS] (Cited on pages 58 and 61 .)

Krall, J., Yurchyshyn, V.B., Slinker, S., Skoug, R.M. and Chen, J., 2006, "Flux Rope Model of the 2003 October 28-30 Coronal Mass Ejection and Interplanetary Coronal Mass Ejection", Astrophys. J., 642, 541-553. [DOI], [ADS] (Cited on page 61.)

Krucker, S., Benz, A.O., Aschwanden, M.J. and Bastian, T.S., 1995, "Location of Type I Radio Continuum and Bursts on YOHKOH Soft X-Ray Maps", Solar Phys., 160, 151-169. [DOI], [ADS] (Cited on page 19.)

Kundu, M.R., Gopalswamy, N., Saba, J.L.R., Schmelz, J.T.S. and Strong, K.T., 1987, "A study of solar preflare activity using two-dimensional radio and SMM-XRP observations", Solar Phys., 114, 273-288. [DOI], [ADS] (Cited on page 20.)

Kuperus, M. and Raadu, M.A., 1974, "The Support of Prominences Formed in Neutral Sheets", Astron. Astrophys., 31, 189. [ADS] (Cited on pages 13 and 39.)

Kurokawa, H., 1987, "Two distinct morphological types of magnetic shear development and their relation to flares", Solar Phys., 113, 259-263. [DOI], [ADS] (Cited on page 14.)

Kusano, K., Maeshiro, T., Yokoyama, T. and Sakurai, T., 2002, "Measurement of Magnetic Helicity Injection and Free Energy Loading into the Solar Corona", Astrophys. J., 577, 501-512. [DOI], [ADS] (Cited on page 38.)

Kusano, K., Maeshiro, T., Yokoyama, T. and Sakurai, T., 2004, "The Trigger Mechanism of Solar Flares in a Coronal Arcade with Reversed Magnetic Shear", Astrophys. J., 610, 537-549. [DOI], [ADS] (Cited on page 25.)

Lantos, P., Kerdraon, A., Rapley, G.G. and Bentley, R.D., 1981, "Relationship between a soft X-ray long duration event and an intense metric noise storm", Astron. Astrophys., 101, 33-38. [ADS] (Cited on page 19.)

Lara, A., Gopalswamy, N., Xie, H., Mendoza-Torres, E., Pérez-Eríquez, R. and Michalek, G., 2006, "Are halo coronal mass ejections special events?", J. Geophys. Res., 111, 6107. [DOI], [ADS] (Cited on page 62.)

Leroy, J.L., 1989, "Observation of prominence magnetic fields", in Dynamics and Structure of Quiescent Solar Prominences, Proceedings of the Workshop, Palma de Mallorca, Spain, November 1987, (Ed.) Priest, E.R., vol. 150 of Astrophysics and Space Science Library, pp. 77-113, Kluwer Academic, Dordrecht. [ADS], [Google Books] (Cited on page 17.)

Leroy, J.L., Bommier, V. and Sahal-Brechot, S., 1983, "The magnetic field in the prominences of the polar crown", Solar Phys., 83, 135-142. [DOI], [ADS] (Cited on pages 13 and 17.)

Li, Y. and Luhmann, J., 2006, "Coronal Magnetic Field Topology over Filament Channels: Implication for Coronal Mass Ejection Initiations", Astrophys. J., 648, 732-740. [DOI], [ADS] (Cited on page 27.) 
Lin, J., 2002, "Energetics and Propagation of Coronal Mass Ejections in Different Plasma Environments", Chin. J. Astron. Astrophys., 2, 539-556. [ADS] (Cited on page 49.)

Lin, J. and Forbes, T.G., 2000, "Effects of reconnection on the coronal mass ejection process", $J$. Geophys. Res., 105, 2375-2392. [DOI], [ADS] (Cited on pages 39, 41, and 49.)

Lin, J., Forbes, T.G., Isenberg, P.A. and Demoulin, P., 1998, "The Effect of Curvature on FluxRope Models of Coronal Mass Ejections", Astrophys. J., 504, 1006. [DOI], [ADS] (Cited on page 34.)

Lin, J., Forbes, T.G. and Isenberg, P.A., 2001, "Prominence eruptions and coronal mass ejections triggered by newly emerging flux", J. Geophys. Res., 106, 25,053-25,074. [DOI], [ADS] (Cited on page 35.)

Lin, J., Li, J., Forbes, T.G., Ko, Y.-K., Raymond, J.C. and Vourlidas, A., 2007, "Features and Properties of Coronal Mass Ejection/Flare Current Sheets", Astrophys. J. Lett., 658, L123-L126. [DOI], [ADS] (Cited on page 50.)

Linker, J.A., Mikić, Z., Riley, P., Lionello, R. and Odstrcil, D., 2003, "Models of Coronal Mass Ejections: A Review with A Look to The Future", in Solar Wind Ten, Proceedings of the Tenth International Solar Wind Conference, Pisa, Italy, 17-21 June 2002, (Eds.) Velli, M., Bruno, R., Malara, F., Bucci, B., vol. 679 of AIP Conference Series, pp. 703-710, American Institute of Physics, Melville, NY. [DOI], [ADS] (Cited on page 12.)

Lites, B.W., 2005, "Magnetic Flux Ropes in the Solar Photosphere: The Vector Magnetic Field under Active Region Filaments", Astrophys. J., 622, 1275-1291. [DOI], [ADS] (Cited on page 14.)

Liu, C., Lee, J., Yurchyshyn, V., Deng, N., Cho, K.-S., Karlický, M. and Wang, H., 2007, "The Eruption from a Sigmoidal Solar Active Region on 2005 May 13", Astrophys. J., 669, 1372-1381. [DOI], [ADS], [arXiv:0707.2240] (Cited on page 18.)

Liu, Y., 2008, "Magnetic Field Overlying Solar Eruption Regions and Kink and Torus Instabilities", Astrophys. J. Lett., 679, L151-L154. [DOI], [ADS] (Cited on page 31.)

Long, D.M., Gallagher, P.T., McAteer, R.T.J. and Bloomfield, D.S., 2008, "The Kinematics of a Globally Propagating Disturbance in the Solar Corona", Astrophys. J. Lett., 680, L81-L84. [DOI], [ADS], [arXiv:0805.2023] (Cited on pages 53 and 54.)

Low, B.C., 1977, "Evolving force-free magnetic fields. I. The development of the preflare stage", Astrophys. J., 212, 234-242. [DOI], [ADS] (Cited on page 25.)

Low, B.C., 1984, "Self-Similar Magnetohydrodynamics. IV. The Physics of Coronal Transients", Astrophys. J., 281, 392. [DOI], [ADS] (Cited on page 58.)

Low, B.C., 1990, "Equilibrium and dynamics of coronal magnetic fields", Annu. Rev. Astron. Astrophys., 28, 491-524. [DOI], [ADS] (Cited on page 58.)

Low, B.C., 1994, "Magnetohydrodynamic processes in the solar corona: Flares, coronal mass ejections, and magnetic helicity", Phys. Plasmas, 1, 1684-1690. [DOI], [ADS] (Cited on page 48.)

Low, B.C., 1996, "Solar Activity and the Corona", Solar Phys., 167, 217-265. [DOI], [ADS] (Cited on pages 17 and 38.)

Low, B.C., 1997, "Solar Activity and the Corona", in Coronal Mass Ejections, (Eds.) Crooker, N., Joselyn, J.A., Feynman, J., vol. 99 of Geophysical Monograph, pp. 39-48, American Geophysical Union, Washington, DC (Cited on pages 12 and 48.) 
Low, B.C., 1999, "Coronal Mass Ejections, Flares and Prominences", in Solar Wind Nine, Proceedings of the Ninth International Solar Wind Conference, Nantucket, MA, 5-9 October 1998, (Eds.) Habbal, S.R., Esser, R., Hollweg, J.V., Isenberg, P.A., vol. 471 of AIP Conference Proceedings, pp. 109-114, American Institute of Physics, Melville, NY. [DOI], [ADS] (Cited on page 10.)

Low, B.C., 2001, "Coronal mass ejections, magnetic flux ropes, and solar magnetism", J. Geophys. Res., 106, 25,141-25,164. [DOI], [ADS] (Cited on pages 36 and 44.)

Low, B.C. and Berger, M.A., 2003, "A Morphological Study of Helical Coronal Magnetic Structures", Astrophys. J., 589, 644-657. [DOI], [ADS] (Cited on page 14.)

Low, B.C. and Hundhausen, J.R., 1995, "Magnetostatic structures of the solar corona. II. The magnetic topology of quiescent prominences", Astrophys. J., 443, 818-836. [DOI], [ADS] (Cited on pages 13 and 14.)

Low, B.C. and Smith, D.F., 1993, "The free energies of partially open coronal magnetic fields", Astrophys. J., 410, 412-425. [DOI], [ADS] (Cited on page 48.)

Low, B.C. and Zhang, M., 2002, "The Hydromagnetic Origin of the Two Dynamical Types of Solar Coronal Mass Ejections", Astrophys. J. Lett., 564, L53-L56. [DOI], [ADS] (Cited on pages 17, 51 , and 52.)

Lynch, B.J., Antiochos, S.K., MacNeice, P.J., Zurbuchen, T.H. and Fisk, L.A., 2004, "Observable Properties of the Breakout Model for Coronal Mass Ejections", Astrophys. J., 617, 589-599. [DOI], [ADS] (Cited on page 26.)

Mackay, D.H. and van Ballegooijen, A.A., 2006, "Models of the Large-Scale Corona. I. Formation, Evolution, and Liftoff of Magnetic Flux Ropes", Astrophys. J., 641, 577-589. [DOI], [ADS] (Cited on page 34.)

MacQueen, R.M. and Fisher, R.R., 1983, "The kinematics of solar inner coronal transients", Solar Phys., 89, 89-102. [DOI], [ADS] (Cited on pages 50 and 59.)

MacTaggart, D. and Hood, A.W., 2009, "Multiple eruptions from magnetic flux emergence", Astron. Astrophys., 508, 445-449. [DOI], [ADS], [arXiv:0910.2616 [astro-ph.SR]] (Cited on page 26.)

Magara, T., Chen, P. F., Shibata, K. and Yokoyama, T., 2000, "A Unified Model of Coronal Mass Ejection-related Type II Radio Bursts", Astrophys. J. Lett., 538, L175-L178. [DOI], [ADS] (Cited on page 46.)

Maia, D., Vourlidas, A., Pick, M., Howard, R., Schwenn, R. and Magalhães, A., 1999, "Radio signatures of a fast coronal mass ejection development on November 6, 1997", J. Geophys. Res., 104, 12,507-12,514. [DOI], [ADS] (Cited on page 6.)

Malherbe, J.M. and Priest, E.R., 1983, "Current sheet models for solar prominences. I. Magnetohydrostatics of support and evolution through quasi-static models", Astron. Astrophys., 123, 80-88. [ADS] (Cited on page 17.)

Malitson, H.H., Feinberg, J. and Stone, R.G., 1973, "A Density Scale for the Interplanetary Medium from Observations of a Type II Solar Radio Burst Out to 1 Astronomical Unit", Astrophys. J. Lett., 183, L35. [DOI], [ADS] (Cited on page 45.) 
Manchester IV, W.B., Gombosi, T.I., Roussev, I., Ridley, A., De Zeeuw, D.L., Sokolov, I.V., Powell, K.G. and Tóth, G., 2004, "Modeling a space weather event from the Sun to the Earth: CME generation and interplanetary propagation", J. Geophys. Res., 109, A02107. [DOI], [ADS] (Cited on pages 25, 31, and 40.)

Manchester IV, W.B., Vourlidas, A., Tóth, G., Lugaz, N., Roussev, I.I., Sokolov, I.V., Gombosi, T.I., De Zeeuw, D.L. and Opher, M., 2008, "Three-dimensional MHD Simulation of the 2003 October 28 Coronal Mass Ejection: Comparison with LASCO Coronagraph Observations", Astrophys. J., 684, 1448-1460. [DOI], [ADS], [arXiv:0805.3707] (Cited on page 62.)

Mancuso, S., Raymond, J.C., Kohl, J., Ko, Y.-K., Uzzo, M. and Wu, R., 2002, "UVCS/SOHO observations of a CME-driven shock: Consequences on ion heating mechanisms behind a coronal shock", Astron. Astrophys., 383, 267-274. [DOI], [ADS] (Cited on page 45.)

Marqué, C., Lantos, P. and Delaboudinière, J.P., 2002, "Multi wavelength investigation of the eruption of a sigmoidal quiescent filament", Astron. Astrophys., 387, 317-325. [DOI], [ADS] (Cited on page 15.)

Martens, P.C.H. and Kuin, N.P.M., 1989, "A circuit model for filament eruptions and two-ribbon flares", Solar Phys., 122, 263-302. [DOI], [ADS] (Cited on page 39.)

Martin, S.F., 1980, "Preflare conditions, changes and events", Solar Phys., 68, 217-236. [DOI], [ADS] (Cited on page 21.)

McAllister, H. and Martin, S.F., 2000, "The Essential Role of Magnetic Reconnection in Erupting Prominences and CMEs", Adv. Space Res., 26, 469-472. [DOI], [ADS] (Cited on page 49.)

McLaughlin, J.A., De Moortel, I., Hood, A.W. and Brady, C.S., 2009, "Nonlinear fast magnetoacoustic wave propagation in the neighbourhood of a $2 \mathrm{D}$ magnetic X-point: oscillatory reconnection", Astron. Astrophys., 493, 227-240. [DOI], [ADS], [arXiv:0901.1781 [astro-ph.SR]] (Cited on page 29.)

Mikić, Z. and Linker, J.A., 1994, "Disruption of coronal magnetic field arcades", Astrophys. J., 430, 898-912. [DOI], [ADS] (Cited on page 25.)

Mikić, Z., Schnack, D.D. and van Hoven, G., 1990, "Dynamical evolution of twisted magnetic flux tubes. I. Equilibrium and linear stability", Astrophys. J., 361, 690-700. [DOI], [ADS] (Cited on page 30.)

Moon, Y.-J., Choe, G.S., Wang, H., Park, Y.D., Gopalswamy, N., Yang, G. and Yashiro, S., 2002, "A Statistical Study of Two Classes of Coronal Mass Ejections", Astrophys. J., 581, 694-702. [DOI], [ADS] (Cited on page 51.)

Moore, R.L. and LaBonte, B.J., 1980, "The filament eruption in the 3B flare of July 29, 1973: Onset and magnetic field configuration", in Solar and Interplanetary Dynamics, Cambridge, Mass., USA, August 27-31, 1979, (Eds.) Sheridan, K.V., Dulk, G.A., vol. 91 of IAU Symposia, pp. 207-210, Reidel, Dordrecht; Boston. [ADS] (Cited on pages 17 and 23.)

Moore, R.L., Sterling, A.C., Hudson, H.S. and Lemen, J.R., 2001, "Onset of the Magnetic Explosion in Solar Flares and Coronal Mass Ejections", Astrophys. J., 552, 833-848. [DOI], [ADS] (Cited on page 24.)

Moreton, G.E. and Ramsey, H.E., 1960, "Recent Observations of Dynamical Phenomena Associated with Solar Flares", Publ. Astron. Soc. Pac., 72, 357. [DOI], [ADS] (Cited on page 54.) 
Moses, D., Clette, F., Delaboudinière, J.-P., Artzner, G.E., Bougnet, M., Brunaud, J., Carabetian, C., Gabriel, A.H., Hochedez, J.F., Millier, F., Song, X.Y., Au, B., Dere, K.P., Howard, R.A., Kreplin, R., Michels, D.J., Defise, J.M., Jamar, C., Rochus, P., Chauvineau, J.P., Marioge, J.P., Catura, R.C., Lemen, J.R., Shing, L., Stern, R.A., Gurman, J.B., Neupert, W.M., Newmark, J., Thompson, B., Maucherat, A., Portier-Fozzani, F., Berghmans, D., Cugnon, P., van Dessel, E.L. and Gabryl, J.R., 1997, "EIT Observations of the Extreme Ultraviolet Sun", Solar Phys., 175, 571-599. [DOI], [ADS] (Cited on page 53.)

Mouschovias, T.C. and Poland, A.I., 1978, "Expansion and broadening of coronal loop transients: A theoretical explanation", Astrophys. J., 220, 675-682. [DOI], [ADS] (Cited on pages 58 and 59.)

Munro, R.H., Gosling, J.T., Hildner, E., MacQueen, R.M., Poland, A.I. and Ross, C.L., 1979, "The association of coronal mass ejection transients with other forms of solar activity", Solar Phys., 61, 201-215. [DOI], [ADS] (Cited on page 52.)

Nakagawa, Y., Wu, S.T. and Tandberg-Hanssen, E., 1975, "Dynamic response of an isothermal static corona to finite-amplitude disturbances", Solar Phys., 41, 387-396. [DOI], [ADS] (Cited on page 58.)

Nakariakov, V.M., Foullon, C., Verwichte, E. and Young, N.P., 2006, "Quasi-periodic modulation of solar and stellar flaring emission by magnetohydrodynamic oscillations in a nearby loop", Astron. Astrophys., 452, 343-346. [DOI], [ADS] (Cited on page 29.)

Nindos, A. and Andrews, M.D., 2004, "The Association of Big Flares and Coronal Mass Ejections: What Is the Role of Magnetic Helicity?", Astrophys. J. Lett., 616, L175-L178. [DOI], [ADS] (Cited on page 38.)

Nitta, N.V. and Hudson, H.S., 2001, "Recurrent flare/CME events from an emerging flux region", Geophys. Res. Lett., 28, 3801-3804. [DOI], [ADS] (Cited on page 19.)

Ohyama, M. and Shibata, K., 1997, "Preflare Heating and Mass Motion in a Solar Flare Associated with Hot Plasma Ejection: 1993 November 11 C9.7 Flare", Publ. Astron. Soc. Japan, 49, 249261. [ADS] (Cited on page 39.)

Ohyama, M. and Shibata, K., 2008, "Hot and Cool Plasmoid Ejections Associated with a Solar Flare", Publ. Astron. Soc. Japan, 60, 85-93. [ADS] (Cited on pages 39 and 58.)

Okamoto, T.J., Nakai, H., Keiyama, A., Narukage, N., UeNo, S., Kitai, R., Kurokawa, H. and Shibata, K., 2004, "Filament Oscillations and Moreton Waves Associated with EIT Waves", Astrophys. J., 608, 1124-1132. [DOI], [ADS] (Cited on page 36.)

Pallavicini, R., Vaiana, G.S., Kahler, S.W. and Krieger, A.S., 1975, "Spatial structure and temporal development of a solar X-ray flare observed from SKYLAB on June 15, 1973", Solar Phys., 45, 411-433. [DOI], [ADS] (Cited on page 19.)

Patsourakos, S., Vourlidas, A., Wang, Y.M., Stenborg, G. and Thernisien, A., 2009, "What Is the Nature of EUV Waves? First STEREO 3D Observations and Comparison with Theoretical Models", Solar Phys., 259, 49-71. [DOI], [ADS], [arXiv:0905.2189] (Cited on page 54.)

Pevtsov, A.A., 2002, "Active-Region Filaments and X-ray Sigmoids", Solar Phys., 207, 111-123. [ADS] (Cited on page 18.)

Phillips, A.D., MacNeice, P.J. and Antiochos, S.K., 2005, "The Role of Magnetic Helicity in Coronal Mass Ejections", Astrophys. J. Lett., 624, L129-L132. [DOI], [ADS] (Cited on page 38.) 
Pneuman, G.W., 1981, "Two-ribbon flares: (post)-flare loops", in Solar Flare Magnetohydrodynam$i c s$, (Ed.) Priest, E.R., vol. 1 of Fluid Mechanics of Astrophysics and Geophysics, pp. 379-428, Gordon and Breach, New York. [ADS], [Google Books] (Cited on page 48.)

Podladchikova, O. and Berghmans, D., 2005, "Automated Detection of EIT Waves and Dimmings", Solar Phys., 228, 265-284. [DOI], [ADS] (Cited on page 57.)

Poland, A.I. and Munro, R.H., 1976, "Interpretation of broad-band polarimetry of solar coronal transients: Importance of $\mathrm{H} \alpha$ emission", Astrophys. J., 209, 927-934. [DOI], [ADS] (Cited on page 58.)

Pomoell, J., Vainio, R. and Kissmann, R., 2008, "MHD Modeling of Coronal Large-Amplitude Waves Related to CME Lift-off", Solar Phys., 253, 249-261. [DOI], [ADS] (Cited on page 54.)

Priest, E.R., 2007, "Solar Atmosphere", in Handbook of the Solar-Terrestrial Environment, (Eds.) Kamide, Y., Chian, A.C.-L., pp. 55-93, Springer, Berlin; New York. [DOI] (Cited on page 32.)

Priest, E.R. and Forbes, T.G., 1990, "Magnetic field evolution during prominence eruptions and two-ribbon flares", Solar Phys., 126, 319-350. [DOI], [ADS] (Cited on page 32.)

Priest, E.R. and Forbes, T.G., 2002, "The magnetic nature of solar flares", Astron. Astrophys. Rev., 10, 313-377. [DOI], [ADS] (Cited on page 5.)

Priest, E.R., Hood, A.W. and Anzer, U., 1989, "A twisted flux-tube model for solar prominences. I. General properties", Astrophys. J., 344, 1010-1025. [DOI], [ADS] (Cited on pages 16 and 17.)

Pulkkinen, T., 2007, "Space Weather: Terrestrial Perspective", Living Rev. Solar Phys., 4, lrsp2007-1. URL (accessed 13 October 2009): http://www.livingreviews.org/lrsp-2007-1 (Cited on page 5.)

Qiu, J. and Yurchyshyn, V.B., 2005, "Magnetic Reconnection Flux and Coronal Mass Ejection Velocity", Astrophys. J. Lett., 634, L121-L124. [DOI], [ADS] (Cited on page 53.)

Qiu, J., Wang, H., Cheng, C.Z. and Gary, D.E., 2004, "Magnetic Reconnection and Mass Acceleration in Flare-Coronal Mass Ejection Events", Astrophys. J., 604, 900-905. [DOI], [ADS] (Cited on page 50 .)

Rachmeler, L.A., DeForest, C.E. and Kankelborg, C.C., 2009, "Reconnectionless CME Eruption: Putting the Aly-Sturrock Conjecture to Rest", Astrophys. J., 693, 1431-1436. [DOI], [ADS], [arXiv:0812.3199] (Cited on pages 49 and 64.)

Ramesh, R. and Sundaram, G.A.S., 2001, "Occurrence of Metric Noise Storms and the Onset of Coronal Mass Ejections in the Solar Atmosphere", Solar Phys., 202, 355-361. [ADS] (Cited on page 19.)

Raulin, J.P. and Klein, K.-L., 1994, "Acceleration of electrons outside flares: Evidence for coronal evolution and height-extended energy release during noise storms", Astron. Astrophys., 281, 536-550. [ADS] (Cited on page 19.)

Raychaudhuri, P., 2005, "Variability of Coronal Mass Ejections", in Coronal and Stellar Mass Ejections, Beijing, China, September 13-17, 2004, (Eds.) Dere, K., Wang, J., Yan, Y., vol. 226 of IAU Symposia, pp. 211-212, Cambridge University Press, Cambridge; New York. [DOI], [ADS], [Google Books] (Cited on page 7.) 
Raymond, J.C., Thompson, B.J., St Cyr, O.C., Gopalswamy, N., Kahler, S., Kaiser, M., Lara, A., Ciaravella, A., Romoli, M. and O'Neal, R., 2000, "SOHO and radio observations of a CME shock wave", Geophys. Res. Lett., 27, 1439-1442. [DOI], [ADS] (Cited on page 45.)

Régnier, S. and Amari, T., 2004, "3D magnetic configuration of the $\mathrm{H} \alpha$ filament and X-ray sigmoid in NOAA AR 8151", Astron. Astrophys., 425, 345-352. [DOI], [ADS] (Cited on page 16.)

Reiner, M.J., Kaiser, M.L., Plunkett, S.P., Prestage, N.P. and Manning, R., 2000, "Radio Tracking of a White-Light Coronal Mass Ejection from Solar Corona to Interplanetary Medium", Astrophys. J. Lett., 529, L53-L56. [DOI], [ADS] (Cited on pages 45 and 46.)

Riley, P., 2007, "An Alternative Interpretation of the Relationship between the Inferred Open Solar Flux and the Interplanetary Magnetic Field", Astrophys. J. Lett., 667, L97-L100. [DOI], [ADS] (Cited on page 12.)

Riley, P., Linker, J.A., Mikić, Z., Odstrcil, D., Zurbuchen, T.H., Lario, D. and Lepping, R.P., 2003, "Using an MHD simulation to interpret the global context of a coronal mass ejection observed by two spacecraft", J. Geophys. Res., 108, 1272. [DOI], [ADS] (Cited on page 40.)

Robbrecht, E., Berghmans, D. and Van der Linden, R.A.M., 2009, "Automated LASCO CME Catalog for Solar Cycle 23: Are CMEs Scale Invariant?", Astrophys. J., 691, 1222-1234. [DOI], [ADS], [arXiv:0810.1252] (Cited on pages 7 and 8.)

Roussev, I.I., Forbes, T.G., Gombosi, T.I., Sokolov, I.V., DeZeeuw, D.L. and Birn, J., 2003, "A Three-dimensional Flux Rope Model for Coronal Mass Ejections Based on a Loss of Equilibrium", Astrophys. J. Lett., 588, L45-L48. [DOI], [ADS] (Cited on page 16.)

Roussev, I.I., Lugaz, N. and Sokolov, I.V., 2007, "New Physical Insight on the Changes in Magnetic Topology during Coronal Mass Ejections: Case Studies for the 2002 April 21 and August 24 Events", Astrophys. J. Lett., 668, L87-L90. [DOI], [ADS] (Cited on pages 11 and 45.)

Roy, J.-R. and Tang, F., 1975, "Slow X-ray bursts and flares with filament disruption", Solar Phys., 42, 425-439. [DOI], [ADS] (Cited on page 19.)

Rust, D.M., 1983, "Coronal disturbances and their terrestrial effects", Space Sci. Rev., 34, 21-36. [DOI], [ADS] (Cited on page 6.)

Rust, D.M., 1994, "Spawning and shedding helical magnetic fields in the solar atmosphere", Geophys. Res. Lett., 21, 241-244. [DOI], [ADS] (Cited on page 38.)

Rust, D.M. and Kumar, A., 1996, "Evidence for Helically Kinked Magnetic Flux Ropes in Solar Eruptions", Astrophys. J. Lett., 464, L199-L202. [DOI], [ADS] (Cited on page 14.)

Rust, D.M., Nakagawa, Y. and Neupert, W.M., 1975, "EUV emission, filament activation and magnetic fields in a slow-rise flare", Solar Phys., 41, 397-414. [DOI], [ADS] (Cited on page 19.)

Sakurai, T., 1976, "Magnetohydrodynamic interpretation of the motion of prominences", Publ. Astron. Soc. Japan, 28, 177-198. [ADS] (Cited on pages 30 and 31.)

Savcheva, A. and van Ballegooijen, A., 2009, "Nonlinear Force-free Modeling of a Long-lasting Coronal Sigmoid", Astrophys. J., 703, 1766-1777. [DOI], [ADS] (Cited on page 16.)

Schmidt, J.M. and Ofman, L., 2010, "Global Simulation of an Extreme Ultraviolet Imaging Telescope Wave", Astrophys. J., 713, 1008-1015. [DOI], [ADS] (Cited on page 54.)

Living Reviews in Solar Physics

http://www. livingreviews.org/lrsp-2011-1 
Schrijver, C.J., DeRosa, M.L., Metcalf, T., Barnes, G., Lites, B., Tarbell, T., McTiernan, J., Valori, G., Wiegelmann, T., Wheatland, M.S., Amari, T., Aulanier, G., Démoulin, P., Fuhrmann, M., Kusano, K., Régnier, S. and Thalmann, J.K., 2008, "Nonlinear Force-free Field Modeling of a Solar Active Region around the Time of a Major Flare and Coronal Mass Ejection", Astrophys. J., 675, 1637-1644. [DOI], [ADS], [arXiv:0712.0023] (Cited on page 15.)

Schuck, P.W., 2010, "The Photospheric Energy and Helicity Budgets of the Flux-injection Hypothesis", Astrophys. J., 714, 68-88. [DOI], [ADS], [arXiv:1003.1647] (Cited on page 30.)

Schwenn, R., 1996, "An Essay on Terminology, Myths and Known Facts: Solar Transient - Flare - CME - Driver Gas - Piston - BDE - Magnetic Cloud - Shock Wave - Geomagnetic Storm", Astrophys. Space Sci., 243, 187-193. [DOI], [ADS] (Cited on page 6.)

Schwenn, R., 2006, "Space Weather: The Solar Perspective", Living Rev. Solar Phys., 3, lrsp2006-2. URL (accessed 13 October 2009):

http://www.livingreviews.org/lrsp-2006-2 (Cited on pages 5, 6, and 44.)

Seehafer, N., 1990, "Electric current helicity in the solar atmosphere", Solar Phys., 125, 219-232. [DOI], [ADS] (Cited on page 38.)

Sheeley Jr, N.R., Walters, J.H., Wang, Y.-M. and Howard, R.A., 1999, "Continuous tracking of coronal outflows: Two kinds of coronal mass ejections", J. Geophys. Res., 104, 24,739-24,768. [DOI], [ADS] (Cited on page 50.)

Sheeley Jr, N.R., Hakala, W.N. and Wang, Y.-M., 2000, "Detection of coronal mass ejection associated shock waves in the outer corona", J. Geophys. Res., 105, 5081-5092. [DOI], [ADS] (Cited on page 45.)

Shibata, K., 1996, "New observational facts about solar flares from Yohkoh studies - Evidence of magnetic reconnection and a unified model of flares", Adv. Space Res., 17, 9-18. [DOI], [ADS] (Cited on page 52.)

Shibata, K., 2003, "Progress on Numerical Simulations of Solar Flares and Coronal Mass Ejections", in Proceedings of the IAU 8th Asian-Pacific Regional Meeting, Volume I, Tokyo, Japan, July $2-5$, 2002, (Eds.) Ikeuchi, S., Hearnshaw, J., Hanawa, T., vol. 289 of ASP Conference Series, pp. 381-388, Astronomical Society of the Pacific, San Francisco. [ADS] (Cited on page 49.)

Shibata, K. and Tanuma, S., 2001, "Plasmoid-induced-reconnection and fractal reconnection", Earth Planets Space, 53, 473-482. [ADS], [arXiv:astro-ph/0101008] (Cited on pages 39 and 40.)

Shibata, K., Ishido, Y., Acton, L.W., Strong, K.T., Hirayama, T., Uchida, Y., McAllister, A.H., Matsumoto, R., Tsuneta, S., Shimizu, T., Hara, H., Sakurai, T., Ichimoto, K., Nishino, Y. and Ogawara, Y., 1992, "Observations of X-ray jets with the YOHKOH Soft X-ray Telescope", Publ. Astron. Soc. Japan, 44, L173-L179. [ADS] (Cited on page 11.)

Shiota, D., Isobe, H., Chen, P.F., Yamamoto, T.T., Sakajiri, T. and Shibata, K., 2005, "SelfConsistent Magnetohydrodynamic Modeling of a Coronal Mass Ejection, Coronal Dimming, and a Giant Cusp-shaped Arcade Formation", Astrophys. J., 634, 663-678. [DOI], [ADS], [arXiv:astroph/0508478] (Cited on page 29.)

Simnett, G.M. and Harrison, R.A., 1985, "The onset of coronal mass ejections", Solar Phys., 99, 291-311. [DOI], [ADS] (Cited on pages 19, 20, and 29.)

Srivastava, N., Schwenn, R., Inhester, B., Martin, S.F. and Hanaoka, Y., 2000, "Factors Related to the Origin of a Gradual Coronal Mass Ejection Associated with an Eruptive Prominence on 1998 June 21-22", Astrophys. J., 534, 468-481. [DOI], [ADS] (Cited on page 19.) 
St Cyr, O.C., Burkepile, J.T., Hundhausen, A.J. and Lecinski, A.R., 1999, "A comparison of ground-based and spacecraft observations of coronal mass ejections from 1980-1989", J. Geophys. Res., 104, 12,493-12,506. [DOI], [ADS] (Cited on pages 42, 44, 51, and 59.)

St Cyr, O.C., Howard, R.A., Sheeley, Jr, N.R., Plunkett, S.P., Michels, D.J., Paswaters, S.E., Koomen, M.J., Simnett, G.M., Thompson, B.J., Gurman, J.B., Schwenn, R., Webb, D.F., Hildner, E. and Lamy, P.L., 2000, "Properties of coronal mass ejections: SOHO LASCO observations from January 1996 to June 1998", J. Geophys. Res., 105(A8), 18,169-18,186. [DOI], [ADS] (Cited on pages 15 and 58.$)$

Steinolfson, R.S. and Nakagawa, Y., 1977, "Dynamical response of the solar corona. III. Numerical simulation of the 1973 June 10 coronal transient", Astrophys. J., 215, 345-355. [DOI], [ADS] (Cited on page 58.)

Sterling, A.C. and Moore, R.L., 2004, "Evidence for Gradual External Reconnection before Explosive Eruption of a Solar Filament", Astrophys. J., 602, 1024-1036. [DOI], [ADS] (Cited on page 27.)

Sterling, A.C. and Moore, R.L., 2005, "Slow-Rise and Fast-Rise Phases of an Erupting Solar Filament, and Flare Emission Onset", Astrophys. J., 630, 1148-1159. [DOI], [ADS] (Cited on page 40.)

Sturrock, P.A., 1966, "Model of the High-Energy Phase of Solar Flares", Nature, 211, 695-697. [DOI], [ADS] (Cited on page 12.)

Sturrock, P.A., 1991, "Maximum energy of semi-infinite magnetic field configurations", Astrophys. J., 380, 655-659. [DOI], [ADS] (Cited on pages 26 and 48.)

Sturrock, P.A., Weber, M., Wheatland, M.S. and Wolfson, R., 2001, "Metastable Magnetic Configurations and Their Significance for Solar Eruptive Events", Astrophys. J., 548, 492-496. [DOI], [ADS] (Cited on pages 23, 37, and 49.)

Su, Y., 2007, Magnetic Shear in Two-ribbon Solar Flares, Ph.D. Thesis, Purple Mountain Observatory, Nanjing. [ADS] (Cited on page 16.)

Švestka, Z. and Cliver, E.W., 1992, "History and Basic Characteristics of Eruptive Flares", in Eruptive Solar Flares, Proceedings of Colloquium no. 133 of the IAU held at Iguazú, Argentina, 2-6 August, 1991, (Eds.) Švestka, Z., Jackson, B.V., Machado, M.E., vol. 399 of Lecture Notes in Physics, p. 1, Springer, Berlin; New York. [DOI], [ADS] (Cited on page 12.)

Tandberg-Hanssen, E., 1974, Solar Prominences, vol. 12 of Geophysics and Astrophysics Monographs, D. Reidel, Dordrecht (Cited on page 36.)

Tang, F., 1987, "Quiescent prominences - Where are they formed?", Solar Phys., 107, 233-237. [DOI], [ADS] (Cited on page 27.)

Tappin, S.J., 1991, "Do all solar flares have X-ray precursors?", Astron. Astrophys. Suppl., 87, 277-302. [ADS] (Cited on page 22.)

Temmer, M., Vršnak, B., Žic, T. and Veronig, A.M., 2009, "Analytic Modeling of the Moreton Wave Kinematics", Astrophys. J., 702, 1343-1352. [DOI], [ADS], [arXiv:0908.3746] (Cited on page 54.)

Thalmann, J.K. and Wiegelmann, T., 2008, "Evolution of the flaring active region NOAA 10540 as a sequence of nonlinear force-free field extrapolations", Astron. Astrophys., 484, 495-502. [DOI], [ADS] (Cited on page 10.)

Living Reviews in Solar Physics

http://www. livingreviews.org/lrsp-2011-1 
Thernisien, A., Vourlidas, A. and Howard, R.A., 2009, "Forward Modeling of Coronal Mass Ejections Using STEREO/SECCHI Data", Solar Phys., 256, 111-130. [DOI], [ADS] (Cited on page 58.)

Thompson, B.J. and Myers, D.C., 2009, "A Catalog of Coronal 'EIT Wave' Transients", Astrophys. J. Suppl. Ser., 183, 225-243. [DOI], [ADS] (Cited on page 53.)

Thompson, B.J., Plunkett, S.P., Gurman, J.B., Newmark, J.S., St Cyr, O.C. and Michels, D.J., 1998, "SOHO/EIT observations of an Earth-directed coronal mass ejection on May 12, 1997", Geophys. Res. Lett., 25, 2465-2468. [DOI], [ADS] (Cited on page 53.)

Thompson, B.J., Gurman, J.B., Neupert, W.M., Newmark, J.S., Delaboudinière, J.-P., St Cyr, O.C., Stezelberger, S., Dere, K.P., Howard, R.A. and Michels, D.J., 1999, "SOHO/EIT Observations of the 1997 April 7 Coronal Transient: Possible Evidence of Coronal Moreton Waves", Astrophys. J. Lett., 517, L151-L154. [DOI], [ADS] (Cited on page 53.)

Thompson, B.J., Cliver, E.W., Nitta, N., Delannée, C. and Delaboudinière, J.-P., 2000, "Coronal Dimmings and Energetic CMEs in April-May 1998", Geophys. Res. Lett., 27, 1431-1434. [DOI], [ADS] (Cited on page 41.)

Titov, V.S. and Démoulin, P., 1999, "Basic topology of twisted magnetic configurations in solar flares", Astron. Astrophys., 351, 707-720. [ADS] (Cited on page 17.)

Titov, V.S., Hornig, G. and Démoulin, P., 2002, "Theory of magnetic connectivity in the solar corona", J. Geophys. Res., 107(A8), 1164. [DOI], [ADS] (Cited on pages 14 and 17.)

Török, T. and Kliem, B., 2005, "Confined and Ejective Eruptions of Kink-unstable Flux Ropes", Astrophys. J. Lett., 630, L97-L100. [DOI], [ADS], [arXiv:astro-ph/0507662] (Cited on pages 30 and 31.)

Török, T. and Kliem, B., 2007, "Numerical simulations of fast and slow coronal mass ejections", Astron. Nachr., 328, 743. [DOI], [ADS], [arXiv:0705.2100] (Cited on pages 35, 49, 53, and 64.)

Tousey, R., 1973, "The Solar Corona", in Space Research XIII, Proceedings of open meetings of working groups on physical sciences of the 15th plenary meeting of COSPAR, Madrid, Spain, 10-24 May, 1972, (Eds.) Rycroft, M.J., Runcorn, S.K., pp. 713-730, Akademie-Verlag, Berlin. [ADS] (Cited on page 5.)

Tripathi, D., Kliem, B., Mason, H.E., Young, P.R. and Green, L.M., 2009, "Temperature Tomography of a Coronal Sigmoid Supporting the Gradual Formation of a Flux Rope", Astrophys. J. Lett., 698, L27-L32. [DOI], [ADS], [arXiv:0904.4782] (Cited on page 15.)

Uchida, Y., 1968, "Propagation of Hydromagnetic Disturbances in the Solar Corona and Moreton's Wave Phenomenon", Solar Phys., 4, 30-44. [DOI], [ADS] (Cited on pages 46 and 54.)

Uchida, Y., 1974, "Behavior of the flare produced coronal MHD wavefront and the occurrence of type II radio bursts", Solar Phys., 39, 431-449. [DOI], [ADS] (Cited on page 46.)

Ugarte-Urra, I., Warren, H.P. and Winebarger, A.R., 2007, "The Magnetic Topology of Coronal Mass Ejection Sources", Astrophys. J., 662, 1293-1301. [DOI], [ADS], [arXiv:astro-ph/0703049] (Cited on page 27.)

Vaiana, G.S., Krieger, A.S. and Timothy, A.F., 1973, "Identification and Analysis of Structures in the Corona from X-Ray Photography", Solar Phys., 32, 81-116. [DOI], [ADS] (Cited on page 41.) 
van Ballegooijen, A.A., 2004, "Observations and Modeling of a Filament on the Sun", Astrophys. J., 612, 519-529. [DOI], [ADS] (Cited on page 16.)

van Ballegooijen, A.A. and Martens, P.C.H., 1989, "Formation and eruption of solar prominences", Astrophys. J., 343, 971-984. [DOI], [ADS] (Cited on pages 24 and 25.)

van Driel-Gesztelyi, L. and Culhane, J.L., 2009, "Magnetic Flux Emergence, Activity, Eruptions and Magnetic Clouds: Following Magnetic Field from the Sun to the Heliosphere", Space Sci. Rev., 144, 351-381. [DOI], [ADS] (Cited on page 13.)

van Driel-Gesztelyi, L., Démoulin, P. and Mandrini, C.H., 2003, "Observations of magnetic helicity", Adv. Space Res., 32, 1855-1866. [DOI], [ADS] (Cited on page 22.)

van Tend, W. and Kuperus, M., 1978, "The development of coronal electric current systems in active regions and their relation to filaments and flares", Solar Phys., 59, 115-127. [DOI], [ADS] (Cited on page 32.)

Veronig, A.M., Temmer, M. and Vršnak, B., 2008, "High-Cadence Observations of a Global Coronal Wave by STEREO EUVI", Astrophys. J. Lett., 681, L113-L116. [DOI], [ADS], [arXiv:0806.0710] (Cited on page 54.)

Veronig, A.M., Muhr, N., Kienreich, I.W., Temmer, M. and Vršnak, B., 2010, "First Observations of a Dome-shaped Large-scale Coronal Extreme-ultraviolet Wave", Astrophys. J. Lett., 716 , L57-L62. [DOI], [ADS], [arXiv:1005.2060] (Cited on page 54.)

Vial, J.-C., Auchère, F., Chang, J., Fang, C., Gan, W.Q., Klein, K.-L., Prado, J.-Y., Trottet, G., Wang, C. and Yan, Y.H., 2007, "SMESE: A SMall Explorer for Solar Eruptions", Adv. Space Res., 40, 1787-1801. [DOI], [ADS] (Cited on page 6.)

Vourlidas, A., Subramanian, P., Dere, K.P. and Howard, R.A., 2000, "Large-Angle Spectrometric Coronagraph Measurements of the Energetics of Coronal Mass Ejections", Astrophys. J., 534, 456-467. [DOI], [ADS] (Cited on page 42.)

Vourlidas, A., Buzasi, D., Howard, R.A. and Esfandiari, E., 2002, "Mass and energy properties of LASCO CMEs", in Solar Variability: From Core to Outer Frontiers, Proceedings of the 10th European Solar Physics Meeting, Prague, Czech Republic, 9-14 September 2002, (Ed.) Wilson, A., vol. SP-506 of ESA Conference Proceedings, pp. 91-94, ESA Publications Division, Noordwijk. [ADS] (Cited on pages 6 and 8.)

Vourlidas, A., Wu, S.T., Wang, A.H., Subramanian, P. and Howard, R.A., 2003, "Direct Detection of a Coronal Mass Ejection-Associated Shock in Large Angle and Spectrometric Coronagraph Experiment White-Light Images", Astrophys. J., 598, 1392-1402. [DOI], [ADS], [arXiv:astroph/0308367] (Cited on pages 45, 46, and 62.)

Vršnak, B., 2008, "Processes and mechanisms governing the initiation and propagation of CMEs", Ann. Geophys., 26, 3089-3101. [DOI], [ADS] (Cited on page 5.)

Vršnak, B. and Cliver, E.W., 2008, "Origin of Coronal Shock Waves (Invited Review)", Solar Phys., 253, 215-235. [DOI], [ADS] (Cited on pages 5, 45, and 46.)

Vršnak, B. and Lulić, S., 2000, "Formation Of Coronal Mhd Shock Waves - I. The Basic Mechanism", Solar Phys., 196, 157-180. [DOI], [ADS] (Cited on page 46.)

Vršnak, B., Warmuth, A., Brajša, R. and Hanslmeier, A., 2002, "Flare waves observed in Helium I $10830 \AA$ A. A link between H $\alpha$ Moreton and EIT waves", Astron. Astrophys., 394, 299-310. [DOI], [ADS] (Cited on page 54.)

Living Reviews in Solar Physics

http://www. livingreviews.org/lrsp-2011-1 
Vršnak, B., Sudar, D. and Ruždjak, D., 2005, "The CME-flare relationship: Are there really two types of CMEs?", Astron. Astrophys., 435, 1149-1157. [DOI], [ADS] (Cited on pages 50, 51, 52, and 53.)

Wagner, W.J. and MacQueen, R.M., 1983, "The excitation of type II radio bursts in the corona", Astron. Astrophys., 120, 136-138. [ADS] (Cited on page 46.)

Wang, H., 2006, "Rapid Changes of Photospheric Magnetic Fields around Flaring Magnetic Neutral Lines", Astrophys. J., 649, 490-497. [DOI], [ADS] (Cited on page 24.)

Wang, H.J., Shen, C.C. and Lin, J., 2009, "Numerical Experiments of Wave-like Phenomena Caused by the Disruption of an Unstable Magnetic Configuration", Astrophys. J., 700, 17161731. [DOI], [ADS], [arXiv:0906.2677] (Cited on page 57.)

Wang, J. and Shi, Z., 1993, "The flare-associated magnetic changes in an active region. II. Flux emergence and cancellation", Solar Phys., 143, 119-139. [DOI], [ADS] (Cited on page 64.)

Wang, J., Zhou, G. and Zhang, J., 2004, "Helicity Patterns of Coronal Mass Ejection-associated Active Regions", Astrophys. J., 615, 1021-1028. [DOI], [ADS] (Cited on page 38.)

Wang, Y. and Zhang, J., 2007, "A Comparative Study between Eruptive X-Class Flares Associated with Coronal Mass Ejections and Confined X-Class Flares", Astrophys. J., 665, 1428-1438. [DOI], [ADS], [arXiv:0808.2976] (Cited on page 9.)

Wang, Y.-M., 2000, "EIT Waves and Fast-Mode Propagation in the Solar Corona", Astrophys. J. Lett., 543, L89-L93. [DOI], [ADS] (Cited on page 54.)

Wang, Y.-M. and Sheeley Jr, N.R., 1999, "Filament Eruptions near Emerging Bipoles", Astrophys. J. Lett., 510, L157-L160. [DOI], [ADS] (Cited on page 19.)

Wang, Y.-M., Sheeley Jr, N.R., Socker, D.G., Howard, R.A., Brueckner, G.E., Michels, D.J., Moses, D., St Cyr, O.C., Llebaria, A. and Delaboudinière, J.-P., 1998, "Observations of Correlated White-Light and Extreme-Ultraviolet Jets from Polar Coronal Holes", Astrophys. J., 508, 899907. [DOI], [ADS] (Cited on pages 7 and 11.)

Warmuth, A., 2010, "Large-scale waves in the solar corona: The continuing debate", Adv. Space Res., 45, 527-536. [DOI], [ADS] (Cited on page 53.)

Warmuth, A. and Mann, G., 2005, "A model of the Alfvén speed in the solar corona", Astron. Astrophys., 435, 1123-1135. [DOI], [ADS] (Cited on page 54.)

Warmuth, A., Vršnak, B., Magdalenić, J., Hanslmeier, A. and Otruba, W., 2004, "A multiwavelength study of solar flare waves. II. Perturbation characteristics and physical interpretation", Astron. Astrophys., 418, 1117-1129. [DOI], [ADS] (Cited on page 54.)

Webb, D.F. and Howard, R.A., 1994, "The solar cycle variation of coronal mass ejections and the solar wind mass flux", J. Geophys. Res., 99(A3), 4201-4220. [DOI], [ADS] (Cited on page 7.)

Webb, D.F. and Hundhausen, A.J., 1987, "Activity associated with the solar origin of coronal mass ejections", Solar Phys., 108, 383-401. [DOI], [ADS] (Cited on page 6.)

Webb, D.F., Krieger, A.S. and Rust, D.M., 1976, "Coronal X-ray enhancements associated with H-alpha filament disappearances", Solar Phys., 48, 159-186. [DOI], [ADS] (Cited on page 19.)

Wen, Y.-Y., Wang, J.-X. and Zhang, Y.-Z., 2007, "Magnetic Properties of Metric Noise Storms Associated with Coronal Mass Ejections", Chin. J. Astron. Astrophys., 7, 265-280. [DOI], [ADS] (Cited on pages 19 and 20.) 
Wild, J.P. and McCready, L.L., 1950, "Observations of the Spectrum of High-Intensity Solar Radiation at Metre Wavelengths. I. The Apparatus and Spectral Types of Solar Burst Observed", Aust. J. Sci. Res. A, 3, 387. [ADS] (Cited on page 45.)

Wills-Davey, M.J. and Attrill, G.D.R., 2009, "EIT Waves: A Changing Understanding over a Solar Cycle", Space Sci. Rev., 149, 325-353. [DOI], [ADS] (Cited on pages 53, 54, and 57.)

Wills-Davey, M.J. and Thompson, B.J., 1999, "Observations of a Propagating Disturbance in TRACE", Solar Phys., 190, 467-483. [DOI], [ADS] (Cited on page 53.)

Wills-Davey, M.J., DeForest, C.E. and Stenflo, J.O., 2007, "Are 'EIT Waves' Fast-Mode MHD Waves?", Astrophys. J., 664, 556-562. [DOI], [ADS], [arXiv:0704.2828] (Cited on pages 54 and 57.)

Wolfson, R. and Dlamini, B., 1997, "Cross-Field Currents: an Energy Source for Coronal Mass Ejections?", Astrophys. J., 483, 961. [DOI], [ADS] (Cited on page 48.)

Wolfson, R. and Low, B.C., 1992, "Energy buildup in sheared force-free magnetic fields", Astrophys. J., 391, 353-358. [DOI], [ADS] (Cited on page 48.)

Wu, S.T., Wang, S., Dryer, M., Poland, A.I., Orwig, L.E., Sime, D.G., Wolfson, C.J. and Maxwell, A., 1983, "Magnetohydrodynamic simulation of the coronal transient associated with the solar limb flare of 1980, June 29, 18:21 UT", Solar Phys., 85, 351-373. [DOI], [ADS] (Cited on page 58.)

Wu, S.T., Zheng, H., Wang, S., Thompson, B.J., Plunkett, S.P., Zhao, X.P. and Dryer, M., 2001, "Three-dimensional numerical simulation of MHD waves observed by the Extreme Ultraviolet Imaging Telescope", J. Geophys. Res., 106, 25,089-25,102. [DOI], [ADS] (Cited on pages 54 and 55.)

Wu, S.T., Zhang, T.X., Tandberg-Hanssen, E., Liu, Y., Feng, X. and Tan, A., 2004, "Numerical Magnetohydrodynamic Experiments for Testing the Physical Mechanisms of Coronal Mass Ejections Acceleration", Solar Phys., 225, 157-175. [DOI], [ADS] (Cited on pages 36 and 51.)

Wu, S.T., Wang, A.H., Liu, Y. and Hoeksema, J.T., 2006, "Data-driven Magnetohydrodynamic Model for Active Region Evolution", Astrophys. J., 652, 800-811. [DOI], [ADS] (Cited on page 23.)

Wu, Y.Q., Tang, Y.H., Dai, Y. and Wu, G.P., 2002, "The Solar Origin of the 6 January 1997 Coronal Mass Ejection", Solar Phys., 207, 159-171. [ADS] (Cited on pages 9 and 53.)

Xu, X.-Y., Fang, C. and Chen, P.F., 2008, "A Statistical Study on the Filament Eruption Caused by New Emerging Flux", Chin. Astron. Astrophys., 32, 56-64. [DOI], [ADS] (Cited on page 29.)

Yan, Y., Deng, Y., Karlický, M., Fu, Q., Wang, S. and Liu, Y., 2001, "The Magnetic Rope Structure and Associated Energetic Processes in the 2000 July 14 Solar Flare", Astrophys. J. Lett., 551, L115-L119. [DOI], [ADS] (Cited on pages 15 and 16.)

Yashiro, S., Gopalswamy, N., Michalek, G. and Howard, R.A., 2002, "Properties of coronal mass ejections and relationship with solar flares", Bull. Am. Astron. Soc., 34, 695. [ADS] (Cited on pages 50 and 53.)

Yashiro, S., Gopalswamy, N., Michalek, G., St Cyr, O.C., Plunkett, S.P., Rich, N.B. and Howard, R.A., 2004, "A catalog of white light coronal mass ejections observed by the SOHO spacecraft", J. Geophys. Res., 109, 7105. [DOI], [ADS] (Cited on pages 6, 7, 8, and 61.) 
Yashiro, S., Gopalswamy, N., Akiyama, S., Michalek, G. and Howard, R.A., 2005, "Visibility of coronal mass ejections as a function of flare location and intensity", J. Geophys. Res., 110, A12S05. [DOI], [ADS] (Cited on page 9.)

Yeh, C.-T., Ding, M.D. and Chen, P.F., 2005, "Kinetic Properties of CMEs Corrected for the Projection Effect", Solar Phys., 229, 313-322. [DOI], [ADS] (Cited on pages 50 and 53.)

Yokoyama, T. and Shibata, K., 2001, "Magnetohydrodynamic Simulation of a Solar Flare with Chromospheric Evaporation Effect Based on the Magnetic Reconnection Model", Astrophys. J., 549, 1160-1174. [DOI], [ADS] (Cited on page 46.)

Yurchyshyn, V., Yashiro, S., Abramenko, V., Wang, H. and Gopalswamy, N., 2005, "Statistical Distributions of Speeds of Coronal Mass Ejections", Astrophys. J., 619, 599-603. [DOI], [ADS] (Cited on page 51.)

Zhang, J. and Dere, K.P., 2006, "A Statistical Study of Main and Residual Accelerations of Coronal Mass Ejections", Astrophys. J., 649, 1100-1109. [DOI], [ADS] (Cited on page 43.)

Zhang, J., Dere, K.P., Howard, R.A., Kundu, M.R. and White, S.M., 2001a, "On the Temporal Relationship between Coronal Mass Ejections and Flares", Astrophys. J., 559, 452-462. [DOI], [ADS] (Cited on pages 9, 18, 42, 43, 52, and 60.)

Zhang, J., Wang, J., Deng, Y. and Wu, D., 2001b, "Magnetic Flux Cancellation Associated with the Major Solar Event on 2000 July 14", Astrophys. J. Lett., 548, L99-L102. [DOI], [ADS] (Cited on pages 19 and 52.)

Zhang, M. and Flyer, N., 2008, "The Dependence of the Helicity Bound of Force-Free Magnetic Fields on Boundary Conditions", Astrophys. J., 683, 1160-1167. [DOI], [ADS], [arXiv:0805.1783] (Cited on page 38.)

Zhang, M. and Low, B. C., 2005, "The Hydromagnetic Nature of Solar Coronal Mass Ejections", Annu. Rev. Astron. Astrophys., 43, 103-137. [DOI], [ADS] (Cited on page 5.)

Zhang, M., Flyer, N. and Low, B.C., 2006, "Magnetic Field Confinement in the Corona: The Role of Magnetic Helicity Accumulation", Astrophys. J., 644, 575-586. [DOI], [ADS], [arXiv:astroph/0603011] (Cited on page 38.)

Zhang, Q.-M., Guo, Y., Chen, P.F., Ding, M.-D. and Fang, C., 2010, "Why are halo coronal mass ejections faster?", Res. Astron. Astrophys., 10, 461-472. [DOI], [ADS], [arXiv:1002.3953] (Cited on page 62.)

Zhang, Y., Wang, J., Attrill, G.D.R., Harra, L.K., Yang, Z. and He, X., 2007, "Coronal Magnetic Connectivity and EUV Dimmings", Solar Phys., 241, 329-349. [DOI], [ADS] (Cited on pages 11 and 54.)

Zhang, Y., Zhang, M. and Zhang, H., 2008, "On the Relationship between Flux Emergence and CME Initiation", Solar Phys., 250, 75-88. [DOI], [ADS] (Cited on pages 19 and 22.)

Zhou, G., Wang, J. and Cao, Z., 2003, "Correlation between halo coronal mass ejections and solar surface activity", Astron. Astrophys., 397, 1057-1067. [DOI], [ADS] (Cited on page 9.)

Zhou, G.P., Wang, J.X., Zhang, J., Chen, P.F., Ji, H.S. and Dere, K., 2006, "Two Successive Coronal Mass Ejections Driven by the Kink and Drainage Instabilities of an Eruptive Prominence", Astrophys. J., 651, 1238-1244. [DOI], [ADS] (Cited on page 36.) 
Zhukov, A.N. and Auchère, F., 2004, "On the nature of EIT waves, EUV dimmings and their link to CMEs", Astron. Astrophys., 427, 705-716. [DOI], [ADS] (Cited on page 53.)

Zhukov, A.N. and Veselovsky, I.S., 2007, "Global Coronal Mass Ejections", Astrophys. J. Lett., 664, L131-L134. [DOI], [ADS] (Cited on pages 11 and 45.)

Zhukov, A.N., Rodriguez, L. and de Patoul, J., 2009, "STEREO/SECCHI Observations on 8 December 2007: Evidence Against the Wave Hypothesis of the EIT Wave Origin", Solar Phys., 259, 73-85. [DOI], [ADS] (Cited on pages 53 and 54.) 